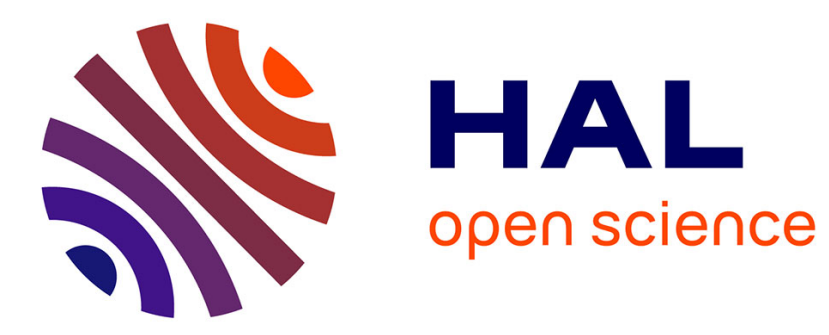

\title{
Robust multisensor time-frequency signal processing: A tutorial review with illustrations of performance enhancement in selected application areas
}

\author{
Boualem Boashash, Abdeldjalil Aissa El Bey
}

\section{- To cite this version:}

Boualem Boashash, Abdeldjalil Aissa El Bey. Robust multisensor time-frequency signal processing: A tutorial review with illustrations of performance enhancement in selected application areas. Digital Signal Processing, 2018, 77, pp.153-186. 10.1016/j.dsp.2017.11.017 . hal-01770948

\author{
HAL Id: hal-01770948 \\ https://hal.science/hal-01770948
}

Submitted on 17 Feb 2022

HAL is a multi-disciplinary open access archive for the deposit and dissemination of scientific research documents, whether they are published or not. The documents may come from teaching and research institutions in France or abroad, or from public or private research centers.
L'archive ouverte pluridisciplinaire HAL, est destinée au dépôt et à la diffusion de documents scientifiques de niveau recherche, publiés ou non, émanant des établissements d'enseignement et de recherche français ou étrangers, des laboratoires publics ou privés. 


\title{
Multisensor Time-Frequency Signal Processing: A tutorial review with illustrations in selected application areas
}

\author{
Boualem Boashash $^{\mathrm{a}, *}$, Abdeldjalil Aïssa-El-Bey ${ }^{\mathrm{a}, \mathrm{b}}$ \\ ${ }^{a}$ Qatar University, Department of Electrical Engineering, Doha, Qatar \\ ${ }^{b}$ IMT Atlantique, UMR CNRS 6285 Lab-STIC, Université Bretagne Loire, Brest, France
}

\begin{abstract}
This tutorial review presents high-resolution multisensor time-frequency distributions (MTFDs) and their application to the analysis of multichannel nonstationary signals. The approach involves combining time-frequency analysis and array signal processing methods. To demonstrate the benefits of MTFDs, this study considers several applications including source localization based on direction of arrival (DOA) estimation and automated component separation (ACS) of non-stationary sources, with particular attention on blind source separation which is a particular case of ACS. The MTFD approach is further illustrated by a new application to EEG signals that specifically uses ACS and DOA estimation methods for artifacts removal and source localization. Supplementary material with code is provided to allow readers to reproduce all the results and apply these methods to their own data.

Keywords: Quadratic TFDs, High-resolution TFDs, Multisensor TFDs, Direction of arrival, Blind source separation, EEG signals, Lead field matrix, Non-stationary array processing, EEG abnormality source localization, Time-frequency analysis
\end{abstract}

${ }^{*}$ Corresponding author 


\section{Contents}

1 Introduction 4

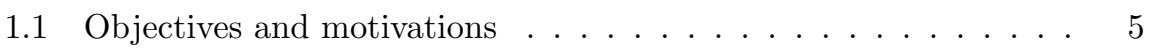

1.1.1 Multichannel systems condition monitoring . . . . . . . 5

1.1.2 Motivation and organization of the paper . . . . . . 7

1.2 Main Objectives . . . . . . . . . . . . . . . . 7

2 Extension of single sensor TFDs to multisensor TFDs 9

2.0 Background and motivation . . . . . . . . . . . . . . 9

2.1 Problem statement . . . . . . . . . . . . . . . . . . . 10

2.1.1 Formulation . . . . . . . . . . . . . . . . 10

2.1.2 Multisensor Time-Frequency Distributions MTFDs . . . . 12

2.1.3 Two types of cross-terms in MTFDs . . . . . . . . . 12

2.2 Mixing models in array processing $\ldots \ldots \ldots \ldots$

2.2 .1 Instantaneous mixing model . . . . . . . . . . . . . 14

2.2 .2 Convolutive mixing model . . . . . . . . . . . . 16

2.3 Non-stationary case array signal model . . . . . . . . . . . . 17

2.3.1 Defining multisensor TFDs . . . . . . . . . . . 17

2.3.2 High resolution MTFDs . . . . . . . . . . . . . . . . 19

2.3.3 Advantages of MTFDs over the Covariance matrix approach 22

2.3.4 Four key properties of MTFDs _ . . . . . . . . . . . 22

2.3.5 Cross-term issues in MTFD . . . . . . . . . . . . 25

2.3 .6 Examples . . . . . . . . . . . . . . 26

3 Blind source separation (BSS) 26

3.0 Background and motivation . . . . . . . . . . . . . 26

3.1 BSS of instantaneous mixtures based on MTFDs . . . . . . . 30

3.1.1 Data whitening preprocessing . . . . . . . . . 31

3.1.2 Source separation by joint diagonalization (JD) . . . . . . 31

3.1.3 Source separation by joint off-diagonalization (JOD) . . . 33

3.1.4 Experiment: Separation of Instantaneous Mixtures . . . . 35 
3.2 BSS of convolutive mixtures based on MTFDs . . . . . . . 35

3.2.1 Signal Model in the convolutive mixture case . . . . . . 35

3.2.2 BSS using MTFD matrices for convolutive mixtures . . . 38

3.2.3 Experiment: Separation of Convolutive Mixtures . . . . . 40

3.3 Under-determined blind source separation (UBSS) . . . . . . . 41

3.3.1 Data model and assumptions . . . . . . . . . . . . . 42

3.3.2 UBSS using Vector Clustering . . . . . . . . . . . . . . . 44

3.3.3 UBSS for non-disjoint sources in the $(t, f)$ domain . . . . 49

3.3.4 Experiment: Underdetermined blind source separation . . 51

3.4 Discussion . . . . . . . . . . . . . . . . . . . 51

3.4.1 Auto-term selection . . . . . . . . . . . . . 52

3.4.2 Number of sources for UBSS clustering step . . . . . . . 52

3.4.3 Number of overlapping sources . . . . . . . . . . . 53

3.4.4 Separation quality versus number of sources . . . . . . . . 54

3.4.5 Overdetermined case . . . . . . . . . . . . . . 55

3.4.6 Improved BSS using high-resolution MTFDs . . . . . . 55

4 Direction of arrival estimation using MTFDs 56

4.0 Background and motivation . . . . . . . . . . . 56

4.1 Time domain DOAs estimation . . . . . . . . . . . 58

4.1.1 Time Domain MUSIC Algorithm . . . . . . . . . . . . . 59

4.1.2 Time Domain ESPRIT Algorithm . . . . . . . . . . 60

4.2 Time-frequency DOAs estimation . . . . . . . . . . . . 61

4.2.1 Time-Frequency MUSIC Algorithm . . . . . . . . . . . 61

4.2.2 Time-Frequency ESPRIT Algorithm . . . . . . . . . . 62

4.3 Examples . . . . . . . . . . . . . . . . 63

4.4 Underdetermined DOAs estimation using MTFDs . . . . . . 64

4.5 Discussion . . . . . . . . . . . . . . . . . . . 66

4.5.1 Signal subspace dimension . . . . . . . . . . . . . . 66

4.5 .2 Spatial resolution . . . . . . . . . . . . . . . 67

4.5.3 Improved DOA estimation using high-resolution MTFDs . 69 
5 Cross Channel Causality Analysis $\quad 70$

5.0 Background and motivation . . . . . . . . . . . 70

5.1 Cross-channel causality and phase synchrony _. . . . . . . 71

5.1.1 Phase synchrony estimation using a complex TFD . . . 73

5.1 .2 Phase synchrony estimation using MTFDs . . . . . . . 75

5.2 Illustrative examples . . . . . . . . . . . . . . . 76

$5.2 .1 \quad$ Experiment $1 \ldots \ldots \ldots \ldots \ldots 77$

$5.2 .2 \quad$ Experiment $2 \ldots \ldots \ldots \ldots \ldots 77$

6 Application: multisensor time-frequency analysis of EEG signals 83

6.1 Data model . . . . . . . . . . . . . . . . . . . 84

6.1.1 Lead Field Matrix . . . . . . . . . . . . . . . . . 84

6.1 .2 Formulation . . . . . . . . . . . . . . . 85

6.2 Application of BSS to EEG artifacts removal . . . . . . . 86

6.2 .0 Background and motivation . . . . . . . . . . 86

6.3 TF-MUSIC applied to source localization of brain EEG abnor-

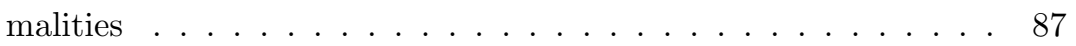

6.3.0 Background and motivation . . . . . . . . . . 87

6.3.1 Source localization of EEG abnormality using TF-MUSIC 87

6.4 Results and discussion . . . . . . . . . . . . . . . . 88

6.4.1 Experiment: application of BSS to EEG artifacts removal 88

6.4.2 Experiment: TF-MUSIC applied to source localization of EEG signals . . . . . . . . . . . . . . . . . . . . . . . . 89

$\begin{array}{lll}7 & \text { Summary and conclusions } & 92\end{array}$

\section{Introduction}

Recent studies have reported significant advances in both array signal processing for non-stationary signals and mutichannel high resolution time-frequency signal processing $[1,2,3,4]$. The main idea of this paper is to combine, extend 
and report these advances in a tutorial review framework and provide corresponding code to allow for reproducible research [5] and application to other areas where multisensor/multichannel data are collected for analysis and decision making.

\subsection{Objectives and motivations}

\subsubsection{Multichannel systems condition monitoring}

In a wide range of engineering applications, a collection or array of measurement sensors is used to solve the problem at hand. The type of sensors depends on the application context. For example, antennas measuring an electrical field are used in telecommunications, while sensors for measuring pressure fluctuations are utilized in acoustic applications such as sonar, ultrasound and speech processing. Regardless of the type of sensor used, by using more than one sensor, one may acquire more information about the measured phenomena.

Typically, the placement of sensors in different physical locations is performed to exploit any spatial diversity present in the signal being measured, and to potentially infer spatial characteristics about the underlying process. For example, in a radar or sonar application, one may wish to determine from which direction an echo is returning, and thus infer the position of a target. In speech processing, it may be desirable to extract the speech signal from a speaker standing in a known position, while suppressing any "noise" coming from other locations, in order to improve intelligibility of the speech in hands-free communications or improve the performance of a speech recognition program. Furthermore, signals can be collected from multisensor systems with a large number of sensors and under several conditions such as low signal to noise ratio $[6,7]$, high interference $[8,9]$, missing data [10] ... etc). Multichannel systems (multi-sensor, sensor array) are used in many applications, such as: biomedical signal processing [11], wireless communications [8, 9], and audio/speech processing $[12,13]$. In such applications, the general objective is to extract critical discriminatory information from the multidimensional signal in order to achieve and/or improve change detection and classification processes 
[14]. Thus, the general approach involves several processing stages such as: acquisition, transformation (time-frequency, time-scale...), information extraction (features, denoising ...etc), clustering, detection and decision.

In many real-life problems, the spectral characteristics of the signals acquired by the multichannel systems are varying with time. This may be a characteristic of the originating process, such as with speech, or due to the surrounding environment, as when the measurement system is in motion with respect to the source of interest. On the one hand, estimation of parameters linked to the time-varying nature of a signal can be enhanced through the use of multiple sensors. This may be relevant, for example, if one wishes to infer the velocity of a moving target with a radar system. On the other hand, if the time-varying nature of the process is known, this property can be used to enhance the estimation of spatial parameters related to the physical location of the signal source; An example is the position of an observed target using radar. It is specifically the synergical combined consideration of both the time-varying characteristics of a measured signal and the spatial information provided by an array of measurement sensors, which is the focus of this paper. A particular formal field of signal processing was motivated by the existence of non-stationary signals, i.e. signals with time-varying spectral characteristics; it is often called joint time-frequency analysis.

Therefore, by combining array signal processing for non-stationary signals and multichannel high resolution time-frequency signal processing, one can provide generic methodologies to a wide field of new applications, such as:

- Abnormalities detection in biomedical signal processing (EEG, ECG...etc) in order to improve early detection of diseases $[14,15,16,17,18,19]$.

- Structural health monitoring of strategic assets such as bridges, dams, for the early detection or prevention of faults [20, 21, 22, 23].

- Energy monitoring for industrial applications, such as electrical consumption monitoring of units in a factory where the objective is to optimize the performance (production versus electrical consumption) [24, 25]. 


\subsubsection{Motivation and organization of the paper}

Previous studies have found that the performance of communication systems, radar, sonar and EEG processing systems could be enhanced by simultaneously taking into account (1) the non-stationary characteristics of measured signals using time-frequency distributions (TFDs) and (2) the spatial information provided by an array of measuring sensors [1]. This results in the development of new methods born from the marriage of these two advanced specialized signal processing fields: hence, the collective name "multisensor time-frequency signal processing". Furthermore, newly developed high-resolution quadratic TFDs have led to improved performance in a wide range of situation $[1,2,14]$. Hence, this study presents a tutorial review on the use of multisensor high-resolution TFDs in the optimal processing of multisensor data e.g. the context of solving array processing problems such as automated component separation (ACS) and direction of arrival estimation (DOA); one key aim being improved resolution, when signals are non-stationary. In addition to resolution, another key aim is the signal causality analysis across sensors/channels and/or signals which allows us to track the time varying location of moving sources by combining spatial and time-frequency information obtained by a multisensor array. For example, (1) in biomedical signal processing, the cross channel causality characterizes the propagation of a seizure location across EEG channels and therefore providing a key information about the time-varying information flow in scalp EEG signals [26]. Combing ACS and DOA should therefore improve decision making from scalp EEG measurements and allow more knowledge about brain activity. (2) In wireless communication, it characterizes the varying spatial location of a moving user (mobile) in cell by exploiting the multiantenna array of the base station. Determining the position and velocity of mobiles in cell is an important issue for cellular networks since the efficient resource allocation depends on it.

\subsection{Main Objectives}

This study aims at presenting and extending past findings in a step by step tutorial review with focus on: 
- Showing the mathematical and physical relationship between the foundations of single variable TFDs and multichannel time-frequency distributions (MTFDs).

- Extending conventional stationary array processing techniques to the nonstationary (time-frequency) array processing case in a step by step tutorial presentation.

- Using advanced algorithms based on multisensor high-resolution TFDs to enhance the capability of multisensor systems in areas such as direction of arrival estimation or separation of non-stationary sources.

- Finally, illustrating the methods developed for multisensor TFDs on new applications such as source localization of brain EEG abnormalities, and propagation path of seizures on the scalp.

The rest of the paper is organized as follows: The extension of single sensor TFDs to multisensor TFDs is discussed in Section 2. Blind source separation methods based on MTFDs are described in Section 3. Then, Section 4 presents a review on direction of arrival estimation algorithms using MTFDs. In Section 5, cross channel causality analysis is introduced with extension to MTFDs. An application of mutisensor time-frequency analysis for EEG signals is provided in Section 6. Finally, Section 7 concludes the paper. In 7, symbols frequently used in this paper are listed in alphabetical order. The meaning in this list should be assumed unless the symbol is otherwise defined in context.

The terminology MTFD is preferred as sensors and therefore channels provide a spatial dimension which is originally discrete, while the $t$ and $f$ variables are naturally continuous. In addition, "M" in "MTFD" can refer to either multisensors or multichannels as the former generates the latter. 


\section{Extension of single sensor TFDs to multisensor TFDs}

\subsection{Background and motivation}

This section aims at reviewing the fundamentals of array processing from a non-stationary perspective in order to establish the mathematical and physical foundation for multisensor TFDs. Next, the advantages of multisensor or multichannel TFDs are discussed.

Multisensor or multichannel TFDs are also called Spatial Time Frequency Distributions (STFDs) in other works. In this study, the terminology MTFD is preferred as discussed in Section 1.2. These techniques can solve array processing problems such as direction of arrival (DOA) estimation with improved resolution, using spatial information for the $(t, f)$ processing of multichannel non-stationary signals.

Many signal processing approaches focus on the case where non-stationary signals are recorded by a single sensor. In fact, in some cases, only one source produces a mono-component signal received by the sensor. However, a single source can also generate a multicomponent signal. In other cases, a different situation arises where several sources generate different components that merge into one signal recorded by one sensor. (See Fig. 1). These two cases are known as "Single Input and Single Output (SISO)" and "Multiple Input and Single Output (MISO)". The TF problem of analyzing multicomponent signals then reduces to a problem of source separation in the case of just one sensor [27]. The traditional field of multisensor (array processing) deals effectively both with this case "Single Input Multiple Output (SIMO)" and the more complex case of multisource and Multi-sensor "Multiple Input and Multiple Output (MIMO)". This section formalized the problem statement for the extension of single sensor TFDs to multisensor TFDs. 


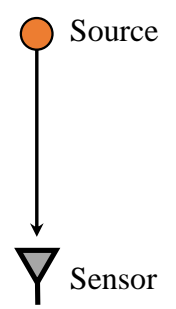

(a)

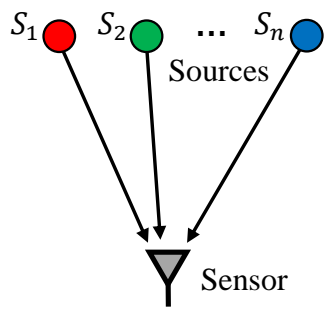

(b)

Figure 1: a) Single-Input Single-Output (SISO) b) Multiple-Input Single-Output (MISO).

\subsection{Problem statement}

Let us consider a non-stationary zero-mean ${ }^{1}$ real signal vector $\mathbf{x}(t)=$ $\left[x_{1}(t), x_{2}(t), \ldots, x_{m}(t)\right]^{T}$ and $\mathbf{z}(t)=\left[z_{1}(t), z_{2}(t), \ldots, z_{m}(t)\right]^{T}$ is the analytic signal associated with the original real signal $\mathbf{x}(t)$ obtained using the Hilbert transform such that:

$$
z_{i}(t)=x_{i}(t)+j \mathcal{H}\left\{x_{i}(t)\right\}, \quad i=1, \ldots, m .
$$

where $\mathcal{H}\{\cdot\}$ represents the Hilbert transform operator defined by:

$$
\mathcal{H}\left\{x_{i}(t)\right\}=\underset{f \rightarrow t}{\mathcal{F}^{-1}}\left\{(-j \operatorname{sgn} f) \underset{t \rightarrow f}{\mathcal{F}}\left\{x_{i}(t)\right\}\right\} .
$$

In the next section, we introduce the formulation of monochannel time-frequency distributions and its extension to the multichannel case.

\subsubsection{Formulation}

In order to introduce the class of multichannel TFDs, we start this section by presenting the foundation of TFDs in the monosensor case ${ }^{2}$. Let us consider a non-stationary monosensor real signal $x(t)$ and its analytic associate $z(t)$.

\footnotetext{
${ }^{1}$ It is assumed, without loss of generality, that $x_{i}(t)$ has zero mean for $i=1, \ldots, m$.

${ }^{2}$ For greater clarity, convenience, and without loss of generality, we focus on the use of quadratic TFDs (QTFDs) as they form a class that encompass most of the useful TFDs used in practice, including the spectrogram and standard filterbank (also called sonogram). Note also that the spectrogram is the square modulus of the short time Fourier transform.
} 
The Wigner-Ville distribution (WVD) is considered as the core distribution of quadratic class of TFDs [1]; it is defined as the Fourier Transform (FT) of the instantaneous auto-correlation function ${ }^{3} K_{z}(t, \tau)$ expressed as:

$$
W_{z}(t, f)=\underset{\tau \rightarrow f}{\mathcal{F}}\left\{K_{z}(t, \tau)\right\}=\int_{\mathbb{R}} K_{z}(t, \tau) e^{-j 2 \pi f \tau} d \tau,
$$

where $K_{z}(t, \tau)$ is defined as

$$
K_{z}(t, \tau)=z\left(t+\frac{\tau}{2}\right) z^{*}\left(t-\frac{\tau}{2}\right)
$$

The WVD defined in Eq. (3) gives optimal concentration for mono-component linear frequency modulation (LFM) signals, but it produces undesirable "artifacts" (or cross-terms) for non-linear frequency modulated (FM) or multicomponent signals. These cross-terms can be minimized by convolving the WVD with a relevant 2D TF kernel; this is expressed as follows:

$$
\rho_{z}(t, f)=W_{z}(t, f) \underset{t f}{* \underset{f}{*}} \gamma(t, f),
$$

where $\rho_{z}(t, f)$ is a quadratic TFD, $\underset{t f}{* *}$ indicates a double convolution and $\gamma(t, f)$ is a $2 \mathrm{D}$ smoothing kernel operating both in $t$ and $f$ variables. The $2 \mathrm{D}$ smoothing of the WVD with $\gamma(t, f)$ can reduce the cross-terms, but it also blurs the autoterms. Therefore, the TF kernels are designed to achieve the best trade-off between minimizing cross-terms and retaining the resolution of auto-terms.

Eq. (5) can be calculated using a time-lag formulation by replacing the convolution in $f$ with a multiplication in lag, yielding the expression:

$$
\rho_{z}(t, f)=\underset{\tau \rightarrow f}{\mathcal{F}}\left\{G(t, \tau) \underset{t}{*} K_{z}(t, \tau)\right\}
$$

where $G(t, \tau)$ is the time-lag kernel of the TFD and is related to $\gamma(t, f)$ by inverse FT (IFT).

$$
G(t, \tau)=\underset{f \rightarrow \tau}{\mathcal{F}^{-1}}\{\gamma(t, f)\}=\int_{\mathbb{R}} \gamma(t, f) e^{j 2 \pi \tau f} d f .
$$

\footnotetext{
${ }^{3}$ The instantaneous auto-correlation function is used to define the instantaneous correlation of signal $z(t)$ for different lags $\tau$ [1, Chapter 2].
} 
Therefore, by extending the previous formulation of time-frequency distributions, the class of multichannel TFDs (MTFDs) for the signal vector $\mathbf{z}(t)$ is then defined as:

$$
\begin{aligned}
\boldsymbol{\rho}_{z z}(t, f) & =\underset{\tau \rightarrow f}{\mathcal{F}}\left\{G(t, \tau) \underset{t}{*} \mathbf{K}_{z z}(t, \tau)\right\} \\
& =\left[\begin{array}{cccc}
\rho_{z_{1}, z_{1}}(t, f) & \rho_{z_{1}, z_{2}}(t, f) & \cdots & \rho_{z_{1}, z_{m}}(t, f) \\
\rho_{z_{2}, z_{1}}(t, f) & \rho_{z_{2}, z_{2}}(t, f) & \cdots & \rho_{z_{2}, z_{m}}(t, f) \\
\vdots & \vdots & \ddots & \vdots \\
\rho_{z_{m}, z_{1}}(t, f) & \rho_{z_{m}, z_{2}}(t, f) & \cdots & \rho_{z_{m}, z_{m}}(t, f)
\end{array}\right],
\end{aligned}
$$

In the above, the time convolution $\underset{t}{*}$ applies on each entry of the spatial instantaneous correlation matrix $\mathbf{K}_{z z}(t, \tau)$ with elements $K_{z_{i}, z_{j}}(t, \tau)=$ $z_{i}\left(t+\frac{\tau}{2}\right) z_{j}^{*}\left(t-\frac{\tau}{2}\right), i, j=1,2, \ldots, m$, being the instantaneous auto-correlation functions, such that:

$$
\begin{aligned}
\mathbf{K}_{z z}(t, \tau)= & \mathbf{z}(t+\tau / 2) \mathbf{z}^{H}(t-\tau / 2) \\
= & {\left[\begin{array}{cccc}
K_{z_{1}, z_{1}}(t, \tau) & K_{z_{1}, z_{2}}(t, \tau) & \cdots & K_{z_{1}, z_{m}}(t, \tau) \\
K_{z_{2}, z_{1}}(t, \tau) & K_{z_{2}, z_{2}}(t, \tau) & \cdots & K_{z_{2}, z_{m}}(t, \tau) \\
\vdots & \vdots & \ddots & \vdots \\
K_{z_{m}, z_{1}}(t, \tau) & K_{z_{m}, z_{2}}(t, \tau) & \cdots & K_{z_{m}, z_{m}}(t, \tau)
\end{array}\right], }
\end{aligned}
$$

\subsubsection{Multisensor Time-Frequency Distributions MTFDs}

In the MTFD matrix $\boldsymbol{\rho}_{z z}(t, f)$ (Eq. (8)), diagonal terms are called autoTFDs and the quadratic class of auto-TFD of $x_{i}(t)$ can be expressed as:

$$
\rho_{z_{i} z_{i}}(t, f)=\int_{-\infty}^{\infty} \int_{-\infty}^{\infty} G(t-u, \tau) z_{i}(u+\tau / 2) z_{i}^{*}(u-\tau / 2) e^{-j 2 \pi \tau f} d u d \tau .
$$

Similarly, the off-diagonal terms are called cross-TFDs. The cross-TFD of two signals $x_{i}(t)$ and $x_{j}(t)$ can be expressed as:

$$
\rho_{z_{i} z_{j}}(t, f)=\int_{-\infty}^{\infty} \int_{-\infty}^{\infty} G(t-u, \tau) z_{i}(u+\tau / 2) z_{j}^{*}(u-\tau / 2) e^{-j 2 \pi \tau f} d u d \tau .
$$

\subsubsection{Two types of cross-terms in MTFDs}

In addition to the auto-TFDs encountered in the monocomponent single channel case, multicomponent or multichannel TF analysis has both auto-TFDs 


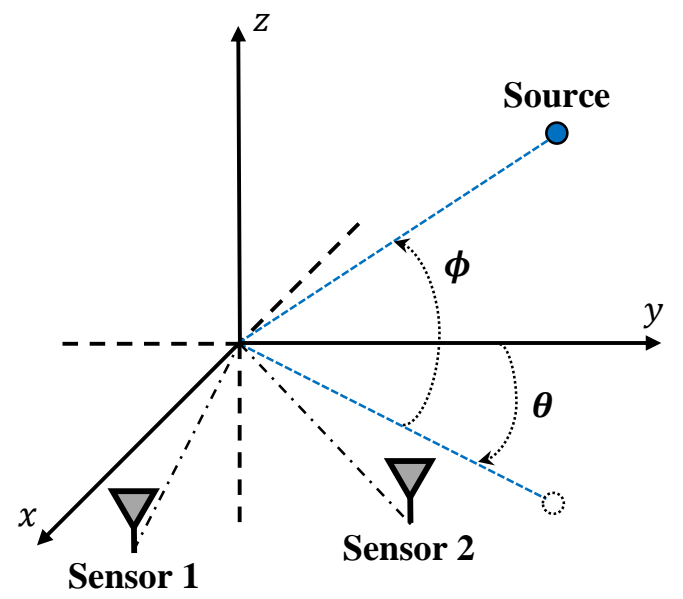

Figure 2: Two sensors and one point source.

and cross-TFDs. There are then two different types of cross-terms in multichannel TFDs. Type 1 is associated with auto-TFDs such cross-terms are formed by the interactions within components of the same source signal; they are located along the auto-terms on the main diagonal of the source TFD matrix. Type 2 cross-terms is generated by cross-TFDs of different source signals from the interactions between signal components of two different sources [3].

\subsection{Mixing models in array processing}

Let us consider a simple example of sensor array with two sensors and one source. Fig. 2 shows the $\theta$ (azimuth) and $\phi$ (elevation) angles of the source. The sources are considered as points in space, from which the propagation of signal energy originates. This may be due, for example, to the emission of electromagnetic energy from a transmitter in a wireless communications system, the reflection of electro-magnetic energy from a target in a radar system, or the reflection of acoustic energy in a sonar system.

The signal energy results from a wave propagating radially outward from the source location. Assuming the far-field scenario, the sources and array are coplanar, implying that the azimuth angle $\theta$ is the only relevant spatial 


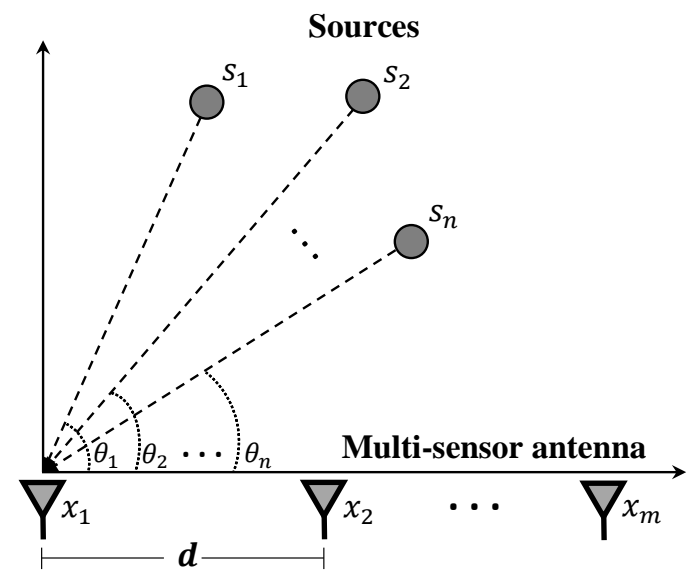

Figure 3: Multi-sensor array and multiple sources.

parameter of a source ${ }^{4}$.

\subsubsection{Instantaneous mixing model}

We introduce in this section the mathematical time domain model of instantaneous mixing systems and present the assumptions under which the instantaneous model is realistic.

The instantaneous mixing system is applicable to a wide range of problems in array signal processing. Typical examples include mixtures speech signals that are simultaneously recorded by several microphones, interfering radio signals arriving at a mobile phone, or brain waves recorded by multiple sensors. Under the assumption that sources are in the far-field, Fig. 3 shows $n$ sources each generating a signal; the combined signals $\left\{s_{1}, s_{2}, \ldots, s_{n}\right\}$ arrive at the sensor that form an $m$ element multisensor array (uniform linear array). The linear data model is given as:

$$
\mathbf{z}(t)=\mathbf{A} \mathbf{s}(t)+\boldsymbol{\eta}(t)
$$

\footnotetext{
${ }^{4}$ If the physical size of the sensor array is very small compared to the distance between the source and sensor array (i.e., the source is in the far-field), the received wavefront may be considered as a plane across the array.
} 


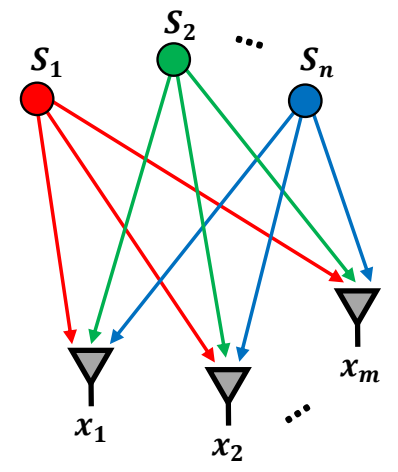

Figure 4: Instantaneous mixing model: $m$ sensors receiving linear combinations of $n$ source signals.

where The $m \times n$ matrix A represents the propagation matrix or mixing matrix, having $n$ column vectors called steering vectors, vector $\mathbf{s}(t)=\left[s_{1}(t), s_{2}(t), \ldots, s_{n}(t)\right]^{T}$ contains the source signals, $\mathbf{z}(t)=$ $\left[z_{1}(t), z_{2}(t), \ldots, z_{m}(t)\right]^{T}$ is the signal vector arriving at the $m$ sensors and $\boldsymbol{\eta}(t)$ represents an additive noise vector whose elements are modeled usually as stationary, temporally and spatially white random processes, and independent of the source signals. In other words, it is assumed that the signals received by an array of sensors (e.g. microphones, antennas, transducers, etc) under farfield assumption form a weighted sum (linear mixture) of the original sources. As illustrated in Fig. 3, the matrix A contains information on the DOAs of the different signals:

$$
\mathbf{A}=\left[\mathbf{a}\left(\theta_{1}\right), \mathbf{a}\left(\theta_{2}\right), \ldots, \mathbf{a}\left(\theta_{n}\right)\right]
$$

where $\mathbf{a}\left(\theta_{i}\right)$ for $i=1,2, \ldots n$ is the steering vector of the array for direction $\theta_{i}$, as illustrated by Figs. 2 and 3 .

Eq. (12) indicates that each sensor receives a combined contribution from each source that forms the observations $\left\{z_{i}(t)\right\}_{i=1}^{m}$. This mixture of $n$ signals is described in Fig 4; the elements $\left\{z_{i}(t)\right\}_{i=1}^{m}$ of vector $\mathbf{z}(t)$ are linear superpositions of the source signals. In the simple case, the sensors receive signals from sources only through a single path (i.e., line-of-sight) and the observa- 


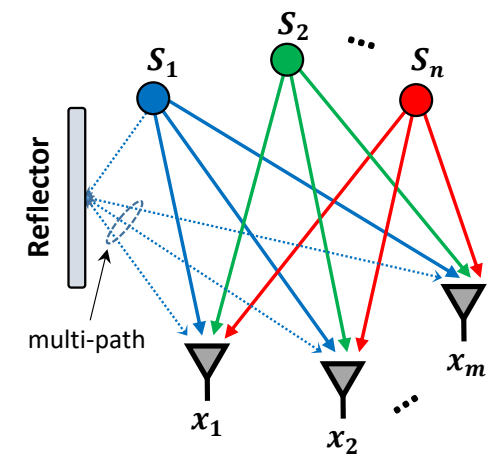

Figure 5: Convolutive mixing model: $m$ sensors receiving linear combinations of delayed (filtered) $n$ source signals.

tions $\left\{z_{i}(t)\right\}_{i=1}^{m}$ depend on the values of $n$ source signals at the same time; this elementary mixing process is referred to as instantaneous mixing.

\subsubsection{Convolutive mixing model}

We introduce in this section the mathematical time domain model of convolutive mixing system. We present the assumptions under which the convolutive model is realistic and some examples.

In the previous section, we have presented an instantaneous mixing model where each recording consists of a sum of differently weighted source signals. The instantaneous mixing model holds under the assumption that sources are in the far-field. However, in many real-life applications, and when the sources are in the near-field, the mixing process is more complex. In such situations, the mixtures are weighted and delayed, and each source contributes to the sum with multiple delays corresponding to the various paths by which a signal propagates to a sensor as shown in Fig. 5. Such filtered sums of different sources form a convolution operation and are therefore called convolutive mixtures. Depending on the situation, the filters may consist of a few delay and attenuation elements, as in radio communications, or possibly a few thousand delay elements as in acoustics. Hence, in such cases the sensor array is clearly convolutive, as the signals picked-up by the sensors consist not only of direct-path signals but they 
are also supplemented by their delayed (reflected) and attenuated versions in the presence of noise. The received signal at the $i^{\text {th }}$ sensor under the convolutive mixing model can be expressed as:

$$
z_{i}(t)=\sum_{j=1}^{n} \sum_{k=0}^{K} a_{i j}(k) s_{j}(t-k)+\eta_{i}(t),
$$

The received signal is a linear mixture of filtered versions of each source signals, and $a_{i j}(k)$ represents filter coefficients between the $i^{\text {th }}$ sensor and the $j^{\text {th }}$ source signal. The filters may be of infinite length with $K \rightarrow \infty$ (and implemented as recursive infinite impulse response systems); in practice it is in general sufficient to assume $K<\infty$. The convolutive model can be formulated in matrix form as:

$$
\mathbf{z}(t)=\sum_{k=0}^{K} \mathbf{A}(k) \mathbf{s}(t-k)+\boldsymbol{\eta}(t),
$$

where the elements of the matrix $\mathbf{A}(k)$ are given by $(\mathbf{A}(k))_{i, j}=a_{i j}(k)$.

\subsection{Non-stationary case array signal model}

Conventional stationary array processing methods are based on the covariance matrix of the received observation at the array of sensors. For nonstationary signals, their spectral content is time varying, and assuming stationarity would be inappropriate and reduce performance. There is therefore a need to extend array processing methods to the non-stationary case in with rigorous formulation of precise relevant models.

\subsubsection{Defining multisensor TFDs}

Multisensor TFDs represent a vector signal corresponding to the number of channels (the space variable). The three dimensions, namely space, time and frequency, are used to construct a matrix called Multisensor Time-Frequency Distribution Matrix as given in Eq. (8). This approach may be called "SpaceTime-Frequency Processing" or more simply "Multisensor Time-Frequency Processing" to account for the fact that the sensor/channel variable is naturally discrete while the $t$ and $f$ variables are naturally continuous at the stage of measurement and prior to sampling. It uses $(t, f)$ domain information across sensors 
located at different spatial locations to characterize the set of non-stationary signals, and their interrelationship. By removing the stationarity assumption, the covariance matrix becomes time-dependent, and is expressed as:

$$
\mathbf{R}_{z z}(t, \tau)=\mathbb{E}\left\{\mathbf{K}_{z z}(t, \tau)\right\}=\mathbb{E}\left\{\mathbf{z}(t+\tau / 2) \mathbf{z}^{H}(t-\tau / 2)\right\},
$$

where $\mathbb{E}\{\cdot\}$ denotes expected value. By assuming the instantaneous mixing model given by Eq. (12) the previous equation can be rewritten as:

$$
\mathbf{R}_{z z}(t, \tau)=\mathbf{A R}_{s s}(t, \tau) \mathbf{A}^{H}+\sigma_{\eta}^{2} \delta(\tau) \mathbf{I}_{m} .
$$

where $\mathbf{R}_{s s}(t, \tau)=\mathbb{E}\left\{\mathbf{s}(t+\tau / 2) \mathbf{s}^{H}(t-\tau / 2)\right\}$ represents the signal sources covariance matrix. The additive noise vector $\boldsymbol{\eta}(t)$ is assumed to be a stationary, temporally and spatially white zero mean random process, such that $\mathbf{R}_{\eta \eta}(t, \tau)=\mathbb{E}\left\{\boldsymbol{\eta}(t+\tau / 2) \boldsymbol{\eta}^{H}(t-\tau / 2)\right\}=\sigma_{\eta}^{2} \delta(\tau) \mathbf{I}_{m}$ where $\delta(t)$ is the Dirac delta function and $\mathbf{I}_{m}$ is the $m \times m$ identity matrix.

In such case, using the extended Wiener-Khintchine theorem, the timevarying power spectrum of a non-stationary signal $\mathbf{z}(t)$ can be estimated as the FT of the filtered time-dependent covariance matrix $\mathbf{R}_{z z}(t, \tau)$,

$$
\boldsymbol{\rho}_{z z}(t, f)=\underset{\tau \rightarrow f}{\mathcal{F}}\left\{G(t, \tau) \underset{t}{*} \mathbf{R}_{z z}(t, \tau)\right\}
$$

Replacing the matrix $\mathbf{R}_{z z}(t, \tau)$ by its expression Eq. (17) yields:

$$
\boldsymbol{\rho}_{z z}(t, f)=\underset{\tau \rightarrow f}{\mathcal{F}}\left\{G(t, \tau) \underset{t}{*}\left(\mathbf{A} \mathbf{R}_{s s}(t, \tau) \mathbf{A}^{H}+\sigma_{\eta}^{2} \delta(\tau) \mathbf{I}_{m}\right)\right\} .
$$

By exploiting the linearity of the Fourier transform and convolution operations, we can rewrite the previous equation as:

$$
\boldsymbol{\rho}_{z z}(t, f)=\mathbf{A} \underset{\tau \rightarrow f}{\mathcal{F}}\left\{G(t, \tau) \underset{t}{*} \mathbf{R}_{s s}(t, \tau)\right\} \mathbf{A}^{H}+\sigma_{\eta}^{2} \mathbf{I}_{m} \underset{\tau \rightarrow f}{\mathcal{F}}\{G(t, \tau) \underset{t}{*} \delta(\tau)\} .
$$

Finally, the time-varying power spectrum of a non-stationary signal $\mathbf{z}(t)$ can be given by:

$$
\boldsymbol{\rho}_{z z}(t, f)=\mathbf{A} \boldsymbol{\rho}_{s s}(t, f) \mathbf{A}^{H}+\sigma^{2} \mathbf{I}_{m}
$$


where $\boldsymbol{\rho}_{s s}(t, f)=\underset{\tau \rightarrow f}{\mathcal{F}}\left\{G(t, \tau) \underset{t}{*} \mathbf{R}_{s s}(t, \tau)\right\}$ is the signal TFD, $\sigma^{2}=\sigma_{\eta}^{2} g(0,0)^{5}$. By replacing the covariance matrices with $\mathbf{R}_{z z}(t, \tau)$ and defining $\boldsymbol{\rho}_{z z}(t, f)$, then the multisensor TFD (i.e., MTFD) matrices, Eq. (17) and Eq. (21) have a similar structure. In Eq. (21), the auto-source TFDs (diagonal entries of $\boldsymbol{\rho}_{s s}(t, f)$ ) and the cross-source TFDs (off-diagonal entries of $\boldsymbol{\rho}_{s s}(t, f)$ ) play an analogous role to the signal auto- and cross-correlations, respectively. This important observation allows one to apply many of the conventional second-order based array processing methods to nonstationary signals by replacing the covariance matrix with the MTFD matrix [3, 28, 29].

\subsubsection{High resolution MTFDs}

This section discusses the use of high resolution TFDs as a basis for designing high-resolution MTFDs and their role in enhancing their performance.

\section{i) Design principle.}

The multisensor MWVD of the multisensor analytic signal $\mathbf{z}(t)$ is defined as the FT of the instantaneous auto-correlation function.

$$
\mathbf{W}_{z z}(t, f)=\underset{\tau \rightarrow f}{\mathcal{F}}\left\{\mathbf{K}_{z z}(t, \tau)\right\}
$$

Despite its many desirable properties, the MWVD has some drawbacks that require a precise handling. It may assume large negative values. Furthermore, it is quadratic in the signal; hence, it exhibits cross-terms. Such cross-terms may be useful in some applications like classification but are undesirable in other applications, including analysis and interpretation as well as multicomponent IF estimation. As in the single sensor case, cross-terms can be reduced by convolving the MWVD with a 2D TF kernel designed specifically for this purpose. By extension of Eq.(5) most quadratic MTFDs, including the Multisensor Spectrogram (MS), can be interpreted as smoothed versions of the MWVD i.e. all

\footnotetext{
${ }^{5} g(\nu, \tau)$ is defined in Eq.(28). For some standard QTFDs $g(0,0)=1$, but this is not always the case. It is a requirement for the marginal property to hold [1].
} 
MTFDs can be written as:

$$
\boldsymbol{\rho}_{z z}(t, f)=\mathbf{W}_{z z}(t, f) \underset{t f}{*} \gamma(t, f),
$$

where $\underset{t f}{* *}$ indicates a double convolution and $\gamma(t, f)$ is a TF kernel filter related to $G(t, \tau)$ by Eq.(7) or equivalently defined by:

$$
\gamma(t, f)=\underset{\tau \rightarrow f}{\mathcal{F}}\{G(t, \tau)\}=\int_{\mathbb{R}} G(t, \tau) e^{-j 2 \pi f \tau} d \tau .
$$

By extending of monosensor case, one obtains an approach for designing and implementing high-performance quadratic MTFDs which is to apply the specification constraints in the dual domain expression i.e. [1, 14]:

$$
\boldsymbol{\rho}_{z z}(t, f)=\underset{\tau \rightarrow f}{\mathcal{F}}\left\{\begin{array}{l}
\mathcal{F}_{\nu \rightarrow t}^{-1} \\
\nu
\end{array}\left\{(\nu, \tau) \mathcal{A}_{z z}(\nu, \tau)\right\}\right\},
$$

where $\mathcal{A}_{z z}(\nu, \tau)$ is the spatial ambiguity function such that:

$$
\begin{aligned}
\mathcal{A}_{z z}(\nu, \tau) & =\underset{t \rightarrow \nu}{\mathcal{F}}\left\{\mathbf{K}_{z z}(t, \tau)\right\} \\
= & {\left[\begin{array}{cccc}
\mathcal{A}_{z_{1}, z_{1}}(\nu, \tau) & \mathcal{A}_{z_{1}, z_{2}}(\nu, \tau) & \cdots & \mathcal{A}_{z_{1}, z_{m}}(\nu, \tau) \\
\mathcal{A}_{z_{2}, z_{1}}(\nu, \tau) & \mathcal{A}_{z_{2}, z_{2}}(\nu, \tau) & \cdots & \mathcal{A}_{z_{2}, z_{m}}(\nu, \tau) \\
\vdots & \vdots & \ddots & \vdots \\
\mathcal{A}_{z_{m}, z_{1}}(\nu, \tau) & \mathcal{A}_{z_{m}, z_{2}}(\nu, \tau) & \cdots & \mathcal{A}_{z_{m}, z_{m}}(\nu, \tau)
\end{array}\right], }
\end{aligned}
$$

with

$$
\mathcal{A}_{z_{i}, z_{j}}(\nu, \tau)=\int_{\mathbb{R}} z_{i}\left(t+\frac{\tau}{2}\right) z_{j}^{*}\left(t-\frac{\tau}{2}\right) e^{-j 2 \pi \nu t} d t
$$

and $g(\nu, \tau)$ is a Doppler-lag kernel defined by:

$$
g(\nu, \tau)=\underset{t \rightarrow \nu}{\mathcal{F}}\{G(t, \tau)\} .
$$

\section{ii) Formulation.}

Separable kernel methods can overcome the resolution limitation of the multisensor spectrogram because they add an additional degree of freedom that controls smoothing along both axes [1, Section 5.7] such that $g(\nu, \tau)=G_{1}(\nu) g_{2}(\tau)$. The S-method [30], extended modified B-distribution (EMBD) [2], compact kernel distribution (CKD) [31] and multidirectional distribution (MDD) [2] are four examples of high resolution separable kernel TFDs used earlier in the single sensor 
case. Previous studies showed that the CKD and the MDD give promising results $[1,2,14]$. For this reason we focus on them for the purpose of illustrating the capability of the MTFD approach. The CKD is defined as:

$$
g(\nu, \tau)=\left\{\begin{array}{ccc}
\exp \left(2 c+\frac{c D^{2}}{\nu^{2}-D^{2}}+\frac{c E^{2}}{\tau^{2}-E^{2}}\right) & \text { if } & |\nu|<D,|\tau|<E \\
0 & \text { otherwise }
\end{array}\right.
$$

The parameters $D$ and $E$ specify the cut-off of the ambiguity domain filters along the $\nu$ and $\tau$ axes. The parameter $c$ controls the shape of the smoothing kernel.

To account for the fact that real signals are multicomponent and can have several directions of energy concentration in the $(t, f)$ domain, the multidirectional kernel (MDK) was formulated as [1, Section 5.9]:

$$
g_{\boldsymbol{\beta}}(\nu, \tau)=\frac{1}{P} \sum_{i=1}^{P} \chi_{\beta_{i}}(\nu, \tau) h_{\beta_{i}}(\nu, \tau),
$$

where $P$ represents the number of branches and the factor $1 / P$ in front of the summation is a normalization coefficient and $\beta_{i}$ is related to the frequency rate $\alpha_{i}$ by $\alpha_{i}=\tan \left(\beta_{i}\right)$. The term $g_{\beta_{i}}(\nu, \tau)$ is the $i^{\text {th }}$ branch of the MDK, which is rotated in the ambiguity domain by an angle $\beta_{i}$,

$$
\chi_{\beta_{i}}(\nu, \tau)= \begin{cases}\exp \left(c_{0}+\frac{c D_{i}^{2}}{F_{\beta_{i}}(\nu, \tau)^{2}-D_{i}^{2}}\right), & \left|F_{\beta_{i}}(\nu, \tau)\right|<D_{i} \\ 0, & \text { otherwise }\end{cases}
$$

where $F_{\beta_{i}}(\nu, \tau)=\cos \left(\beta_{i}\right) \nu-\sin \left(\beta_{i}\right) \tau, c_{0}$ and $c$ are slope-adjustment parameters, and $D_{i}$ is the half-support of $g_{\beta_{i}}(\nu, \tau)$ along the direction perpendicular to the $i^{\text {th }}$ branch of the MDK, and $h_{\beta_{i}}(\nu, \tau)$ is the Doppler lag window for the $i^{\text {th }}$ branch of the MDK; that is,

$$
h_{\beta_{i}}(\nu, \tau)= \begin{cases}\exp \left(c+\frac{c_{0} E_{i}^{2}}{G_{\beta_{i}}(\nu, \tau)^{2}-E_{i}^{2}}\right), & \left|G_{\beta_{i}}(\nu, \tau)\right|<E_{i} \\ 0, & \text { otherwise }\end{cases}
$$

where $G_{\beta_{i}}(\nu, \tau)=\sin \left(\beta_{i}\right) \nu+\cos \left(\beta_{i}\right) \tau$ and $E_{i}$ is related to either the time duration of the LFM components or the bandwidth of spike components. 


\subsubsection{Advantages of MTFDs over the Covariance matrix approach}

This MTFD-based approach is in essence designed to increase the effective SNR. It provides improved robustness with respect to noise by spreading the noise power while simultaneously localizing the source signal power in the $(t, f)$ domain [3]. More precisely, the TFD of white noise is spread over the whole $(t, f)$ plane, while the TFDs of FM like sources are in general confined to much smaller regions, as illustrated in Fig. 6. Let us consider three sources 1, 2 and 3 arriving on a multisensor array. Source 1 occupies the $(t, f)$ region $R_{1}$, Source 2 the $(t, f)$ region $R_{2}$ and Source 3 the $(t, f)$ region $R_{3}$. The $(t, f)$ region characteristics (signatures) of the three sources intersect (i.e., region $R_{12}, R_{13}$, $R_{23}$ and region $\left.R_{123}\right)$, but each source still has its own particular $(t, f)$ region that has no overlap with other sources. On the other hand, the noise is spread over $R_{1}, R_{2}$ and $R_{3}$, as well as the complement region $R_{c}$. When we select $(t, f)$ points for averaging that are within the noise only region $R_{c}$ (such as $\left(t_{1}, f_{1}\right)$ ), then no useful information about the sources is available. But, if we constrain

the selection of $(t, f)$ points to $R_{1}, R_{2}$ or $R_{3}$, such as $\left(t_{2}, f_{2}\right)$, then only the noise contribution in these regions is counted. The effect of removing the $(t, f)$ points that are outside the $(t, f)$ signatures region of the signal arrivals is to increase the SNR. To be more specific, the MTFDs property of concentrating the input signal energy in its instantaneous bandwidth (IB) and around its instantaneous frequency $(\mathrm{IF})$ while distributing the noise over the whole $(t, f)$ plane improves the effective SNR which is important in many applications. A key point is therefore the selection of $(t, f)$ points in the region of interest.

\subsubsection{Four key properties of MTFDs}

Four key advantages results from using array signal processing with MTFD. To explain these advantages, let us use the example presented in the previous section and illustrated by Fig. 6 .

1. The prior knowledge about any time-domain parameters or the type of time-varying frequency behavior of the sources of interest can allow us to directly select the $(t, f)$ regions used in Eq. (21). For example, recall that, 


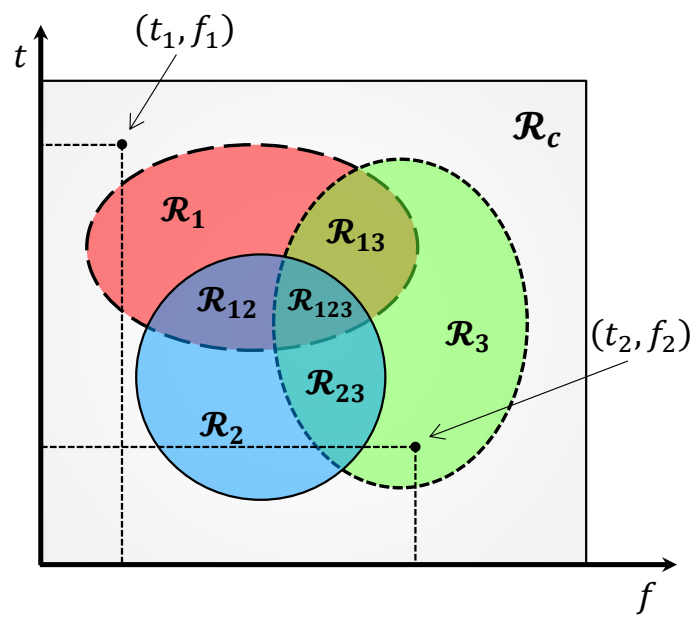

Figure 6: Depiction of three source signals having $(t, f)$ signatures located on different regions.

in the $(\nu, \tau)$ ambiguity domain, all tones map to the time-lag axis. By only including the points on this axis, one can separate and localize all narrowband signals in broadband communications platforms.

2. Eq. (21) is valid for all $(t, f)$ points. Direction finding techniques require $\boldsymbol{\rho}_{z z}(t, f)$ to be full rank; i.e. $\operatorname{Rank}\left(\boldsymbol{\rho}_{z z}\right)=m$, preferably diagonal [3]. On the other hand, blind source separation techniques require the diagonal structure of the same matrix without degenerate eigenvalues (we mean by degenerate eigenvalues that the matrix is rank deficient). These properties combined with high SNR requirements may be difficult to verify using one single $(t, f)$ point. Two different techniques can be used to integrate several $(t, f)$ points into Eq. (21). One uses a simple averaging performed over the signatures of the relevant sources, and the second combines several desired $(t, f)$ points into joint diagonalization or joint block-diagonalization schemes (details are given in Section 3.1 and Section 3.2).

3. The TFD of white noise is spread over the whole $(t, f)$ plane, while the TFDs of the source signals are generally confined to much smaller regions. Fig. 6 indicated that the noise is distributed over $R_{1}, R_{2}$ and $R_{3}$ and the complement region $R_{c}$. When the $(t, f)$ points used in either the joint 
diagonalization or the averaging procedures belong to the noise-only region $R_{c}$, then it means that in this case, no information about the arriving signals is used, and therefore no source localization and signal separation operation can be reasonably achieved. However, if all $(t, f)$ points in Fig. 6 are used, and the selected TFD verifies the marginals, then it follows that the signal average power only is considered. As a consequence, the problem reduces to the second-order covariance based matrix approach used in standard high resolution DOA estimation. This key property means that conventional techniques then become particular cases of the $(t, f)$ array signal processing approach. Finally, if we restrict the $(t, f)$ points to be in the regions $R_{1}, R_{2}$ and $R_{3}$, then only the contribution of the noise in these regions is relevant. Removing the points $(t, f)$ that are not within the $(t, f)$ signatures area of the signal arrivals enhances the input SNR; this can then be used by source localization and signal separation algorithms.

4. If we select only $(t, f)$ points that are within the $(t, f)$ signature of a particular source, then this source is the only one considered by Eq. (21). Such selection, in essence, implicitly performs spatial filtering and removes other sources from consideration. However, such removal does not reduce the number of degrees-of-freedom (DOFs), as it does in beamspace processing [32]. Then, the spatial information conserved which keep the problem as a sensor space processing with the same original number of DOFs unchanged. This finding represents a key contribution of TFDs to direction finding and DOA estimation applications. It is intuitively expected that an antenna array can localize a number of sources equal or even greater than its number of sensors; this is an undertermined case and it is discussed in Sections 3.3 and 4.4. The key condition is that $(t, f)$ regions exist over which the respective $(t, f)$ signatures regions of the sources do not overlap. Let us consider the case of two sensors (see Fig. 6), if all $(t, f)$ points used in direction finding belong to region $R_{1}$ and not $R_{2}$, then the signal subspace defined by Eq. (21) has dimension 1. This concept will be further elaborated in Section 3.3. Thus, by excluding source 2, a noise 
subspace is established. This allows us to proceed with high resolution techniques for localization of source 1. In a general context, one can localize one source at a time or a set of selected sources, depending on the array size, overlapping and distinct $(t, f)$ regions, and the dimension of the noise subspace necessary to achieve the required resolution performance. The same concepts and advantages of $(t, f)$ point selection discussed above for direction finding can be applied to blind source separation problems.

\subsubsection{Cross-term issues in MTFD}

In the case of a single sensor, there are two sources of crossterms. The first type are crossterms that result from the interactions between components of the same source signal. The second type of crossterms are produced from interactions between pairs of signal components belonging to different sources. This second category of crossterms originates from cross-TFDs of the source signals and, at any given $(t, f)$ point, it constitutes the off-diagonal entries of the source TFD matrices $\boldsymbol{\rho}_{z z}(t, f)$ defined in Eq.(21). Although the off-diagonal elements do not necessarily affect the full-rank matrix property required for direction-finding [28], they violate the key assumption in the problem of source separation regarding the diagonal structure of the source TFD matrix. One needs therefore to select the $(t, f)$ points that are in autoterm regions where crossterm contributions are at minimum, e.g., by using a priori information from the source signals.

Note that the method of spatial averaging of the MTFD described in [29] does not reduce the crossterms as in the case with reduced-interference distribution kernels (see Section 2.3.2). Instead, it moves them from their locations on the off-diagonal matrix entries to be part of the matrix diagonal elements. The other parts of the matrix diagonal elements represent the contribution of autoterms at the same point. Therefore, one can set the off-diagonal elements of the source TFD matrix to zeros, and also improve performance by selecting the $(t, f)$ points of peak values, whether these points belong to autoterm or crossterm regions. 


\subsubsection{Examples}

In this illustration, three synthetic signals are generated with sampling frequency $1 \mathrm{~Hz}$ such that:

$$
\begin{aligned}
& z_{1}(t)=\exp (-j 2 \pi(0.25 t+0.012 \cos (3.8 \pi t))), \\
& z_{2}(t)=\exp (-j 2 \pi(0.3) t) \\
& z_{3}(t)=\exp \left(-j 2 \pi\left(0.4 t-\frac{0.3}{256} t^{2}\right)\right)
\end{aligned}
$$

Two MTFDs for the three generated signals are computed using the MWVD and the CKD distributions respectively, as depicted in Figs. 7 and 8. The autoMTFDs in the MWVD diagonal plots in Fig. 7 illustrate the ideal representation of the WVD for mono-component LFM signals, and the deleterious effect of inner cross-terms when representing nonlinear FM signals. Furthermore, the cross-MTFDs in the MWVD off-diagonal plots in Fig. 7 do not represent the intersections between the synthetic signals time-frequency signatures. On the other hand, auto-MTFDs in the CKD, diagonal plots in Fig. 8, illustrate the tradeoff between resolution and cross-terms suppression, when representing different classes of signals. In addition, the cross-MTFD in the CKD off-diagonal plots in Fig. 8 successfully represent the intersections between the synthetic signals time-frequency signatures.

The above results can be reproduced using the codes provided in [5].

\section{Blind source separation (BSS)}

\subsection{Background and motivation}

The aim of automated component separation (ACS) methods is to process the observation acquired by multisensor arrays in such a way that the original unknown source signal can be extracted. The scientific community used the word "blind" to denote all identification or inversion methods that are based on output observations only. Therefore, in the following, we use blind source separation (BSS) to introduce the presented algorithm. 

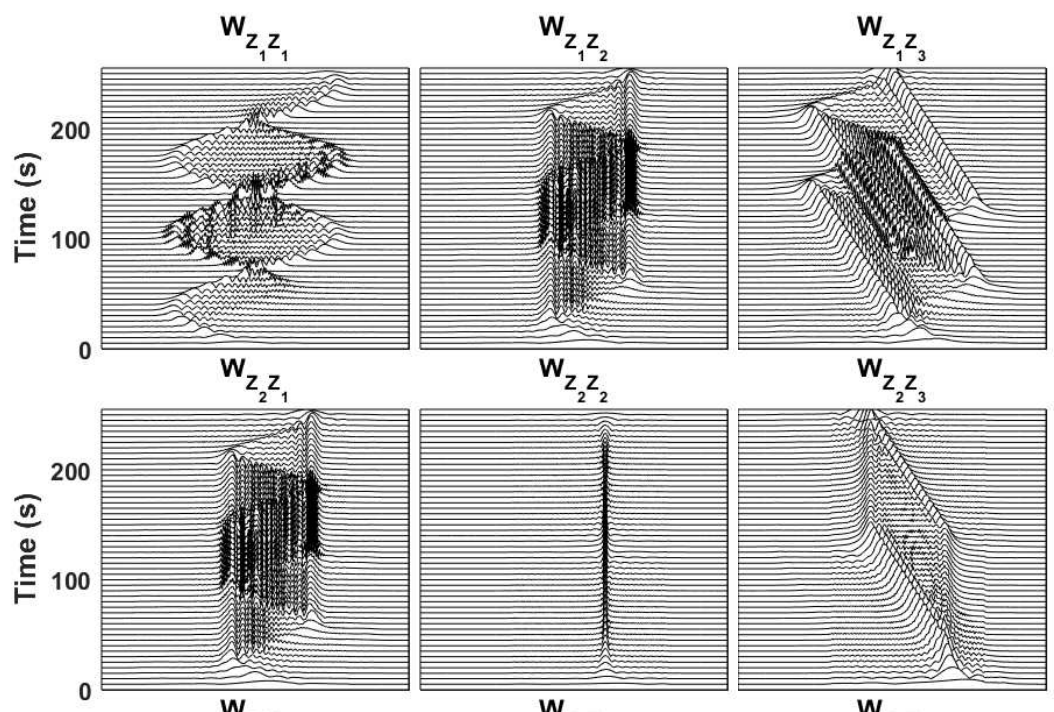

$w_{z_{2} z_{3}}$
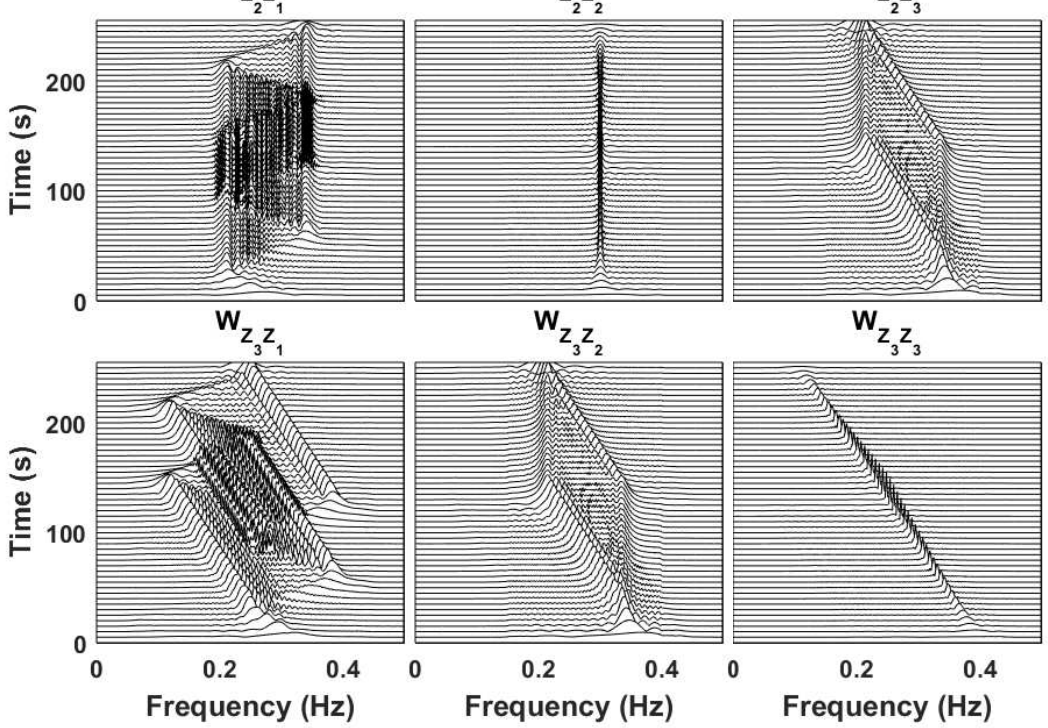

Figure 7: Multisensor Wigner-Ville distribution (MWVD).

This section focuses on the use of modern BSS techniques in applications where its needed to separate different components comprising a signal. BSS applications include: source localization and tracking by radar/sonar systems; multiuser detection in telecommunication; speaker separation (cocktail party problem); biomedical data processing (e.g., separating EEG or ECG signals from artifacts); industrial condition monitoring and fault detection; generally speaking extracting key meaningful features from recorded data, etc. BSS algorithms are used e.g. in EEG applications to localize abnormal EEG sources and 

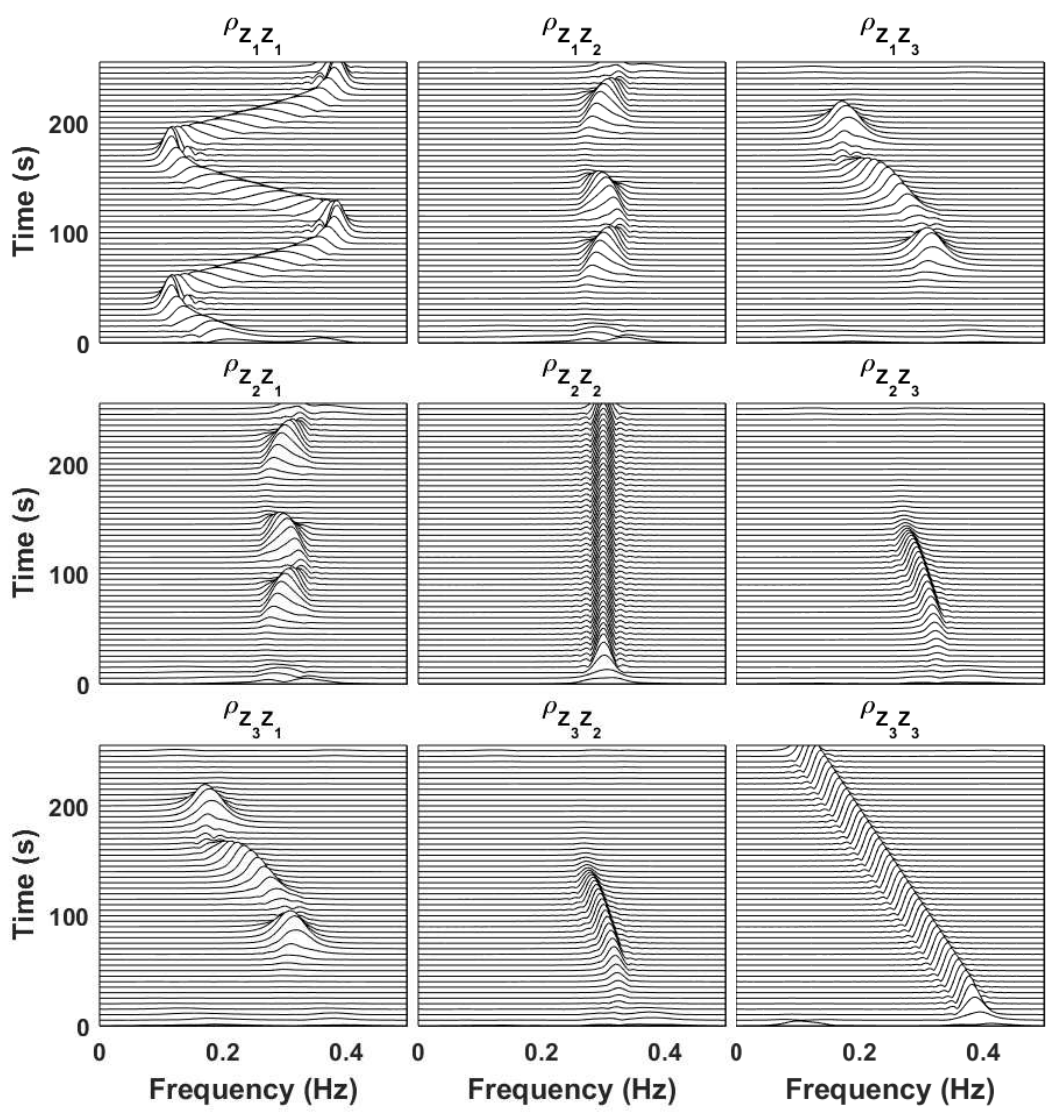

Figure 8: Multisensor compact kernel distribution (CKD): (Tradeoff between resolution and cross-terms suppression).

remove artifacts from the EEG. BSS methods can be divided in two classes: 1) Over-determined case and 2) Under-determined case.

The underlying BSS model assumes that $n$ 'statistically' independent signals are observed through $m$ (possibly noisy) mixtures. Neither the structure of the mixtures nor the source signals are known to the observer. In such a context, the problem is to identify and then disassemble the mixtures blindly.

It turns out that, this apparently difficult problem has elegant solutions 


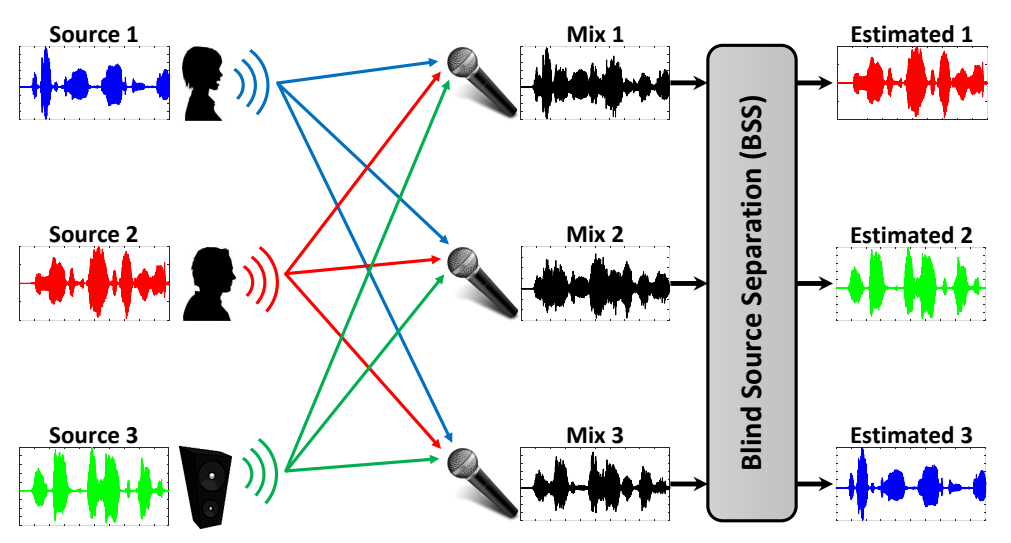

Figure 9: Principle of blind source separation.

that vary according to the type of mixture and the nature of source statistical information. Most BSS approaches, such as independent component analysis (ICA) [33], assume that each source signal is statistically independent from each other. In this context, BSS works only if at most one of the sources has a Gaussian distribution ${ }^{6}$. If each source sequence is a temporally correlated stationary process, BSS works if the source signals have different spectra [3, 34].

The approach is to account for the signal non-stationarity by using a timefrequency approach with BSS so that one can separate and recover the individual incoming signals. The problem can be viewed as a signal synthesis from the $(t, f)$ domain with the constraint of the spatial diversity provided by the multisensor information. One advantage in combining BSS with a TF approach is that the effect of distributing the noise power while simultaneously localizing the source signal energy in the $(t, f)$ domain result in improving the SNR, therefore improving robustness. This TF based BSS methodology includes $(i)$ the BSS problem of instantaneous mixtures and (ii) the general case of BSS of convolutive mixtures.

\footnotetext{
${ }^{6}$ The BSS methods based on the assumption of statistical independence of source signals aim to maximize the non-Gaussianity of estimated sources. It follows that, if all source signals are Gaussian, this BSS methods will not work.
} 
Although BSS algorithms exist in great profusion, the underdetermined case (with number of sensors smaller than number of sources) is less addressed than the overdetermined case (with number of sensors greater than or equal to number of sources). In the underdetermined BSS (UBSS) case, one way to deal with the lack of information is to exploit the assumption that the non-stationary sources are disjoint in the time-frequency domain in order to solve the UBSS problem without prior knowledge on the source distribution.

\subsection{BSS of instantaneous mixtures based on MTFDs}

In this section, we review the BSS technique based on multisensor timefrequency analysis for instantaneous mixing system. Let us consider an $n$ dimensional vector $\mathbf{s}(t)=\left[s_{1}(t), \ldots, s_{n}(t)\right]^{T}$ that represents $n$ non-stationary source signals $s_{i}(t), i=1, \ldots, n$. The $s_{i}(t)$ propagate through a medium and arrives at an array of $m$ sensors which records a mixture of signals described by an $m$-dimensional vector $\mathbf{z}(t)=\left[z_{1}(t), \ldots, z_{m}(t)\right]^{T}$. Therefore, the data model given in Section 2.2.1 by Eq.(12) is applicable in this situation so that $\mathbf{z}(t)=\mathbf{A} \mathbf{s}(t)+\boldsymbol{\eta}(t)$.

A number of BSS algorithms have been developed for the instantaneous mixing case, which make use of the MTFD matrices discussed in the previous section. [35, 36]. The various approches all exploit the underlying diagonal or off-diagonal structure of MTFD matrices at some locations in the $(t, f)$ domain. BSS is achieved by first constructing a set of MTFD matrices, followed by joint diagonalization (JD), joint off-diagonalization (JOD) or combined JD/JOD, to estimate the mixing matrix. The optimization of JD/JOD criteria is based on both orthogonal [35] and non-orthogonal [37] constraints. Such an algorithm is illustrated in Fig. 10.

The principle of BSS based on orthogonal JD/JOD of MTFDs matrices is outlined below [3]. This approach constrains the mixing matrix to be orthogonal, but this is not the case in general. A whitening step needs therefore to be applied to the signals, to verify the orthogonality constraint. 


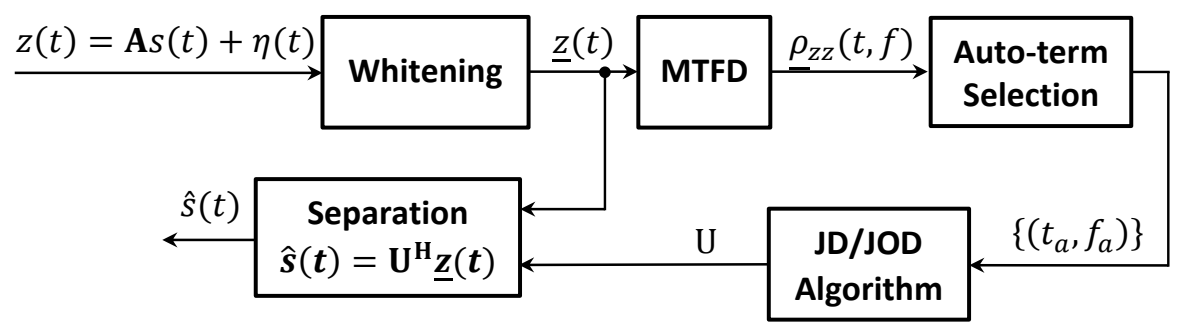

Figure 10: Diagram of the BSS algorithm for instantaneous mixtures based on MTFDs.

\subsubsection{Data whitening preprocessing}

Let us consider an $n \times m$ matrix $\mathbf{H}$ which verifies $(\mathbf{H A})(\mathbf{H A})^{H}=\mathbf{I}_{n}$. Here, $\mathbf{U}=\mathbf{H A}$ is a $n \times n$ unitary whitening matrix (which whitens the signal part of the observations). Then, the whitened MTFD matrices are computed by applying the whitening matrix $\mathbf{H}$ as follow:

$$
\underline{\boldsymbol{\rho}}_{z z}(t, f)=\mathbf{H} \boldsymbol{\rho}_{z z}(t, f) \mathbf{H}^{H}
$$

From the definition of $\mathbf{H}$ and Eq.(21), one can express $\underline{\boldsymbol{\rho}}_{z z}(t, f)$, in the noiseless case, as:

$$
\underline{\boldsymbol{\rho}}_{z z}(t, f)=\mathbf{U} \boldsymbol{\rho}_{s s}(t, f) \mathbf{U}^{H} .
$$

The whitening matrix $\mathbf{H}$ can be estimated in different ways. One example is the inverse square root of the observation autocorrelation matrix; another is to calculate it using the MTFD matrices [3].

\subsubsection{Source separation by joint diagonalization (JD)}

Selecting only auto-term $(t, f)$ points reduces the whitened MTFD matrices to the formulation below:

$$
\underline{\boldsymbol{\rho}}_{z z}^{\mathbf{a}}(t, f)=\mathbf{U} \boldsymbol{\rho}_{s s}^{\mathbf{a}}(t, f) \mathbf{U}^{H}
$$


where $\boldsymbol{\rho}_{s s}^{\mathbf{a}}(t, f)$ is diagonal ${ }^{7}$. Eq.(33) shows that any whitened MTFD matrix is diagonal in the basis formed by the columns of the matrix $\mathbf{U}$ (given that the eigenvalues of $\underline{\boldsymbol{\rho}}_{z z}^{\mathrm{a}}(t, f)$ are the diagonal entries of $\left.\boldsymbol{\rho}_{s s}(t, f)\right)$.

In the case where, for a given $(t, f)$ point, the diagonal elements of $\boldsymbol{\rho}_{s s}^{\mathrm{a}}(t, f)$ are all different, the missing unitary matrix $\mathbf{U}$ may be uniquely retrieved by computing the eigendecomposition of $\underline{\boldsymbol{\rho}}_{z z}^{\mathbf{a}}(t, f)$, up to permutation and scaling ambiguity. Indeed, the BSS problem has an inherent ambiguity concerning the order and amplitudes of the sources. In the case of degenerate eigenvalues indeterminacy occurs, where we mean by degenerate eigenvalues that the matrix $\underline{\boldsymbol{\rho}}_{z z}^{\mathrm{a}}(t, f)$ is rank deficient. Formally, this occurs when $\rho_{s_{i} s_{i}}(t, f)=\rho_{s_{j} s_{j}}(t, f)$, $i \neq j$. One cannot see how to a priori choose the $(t, f)$ point such that the diagonal entries of $\boldsymbol{\rho}_{s s}^{\mathbf{a}}(t, f)$ are all different. Furthermore, if some eigenvalues of $\underline{\boldsymbol{\rho}}_{z z}^{\mathbf{a}}(t, f)$ are degenerate, the robustness of determining $\mathbf{U}$ from the eigendecomposition of a single whitened MTFD matrix suffers. The situation is more appropriate if one considers the joint diagonalization of a combined set $\left\{\underline{\boldsymbol{\rho}}_{z z}^{\mathbf{a}}\left(t_{i}, f_{i}\right) \mid i=1, \ldots, p\right\}$ of $p$ (source auto-term) MTFD matrices. This is equivalent to including several $(t, f)$ points in the source separation problem which decreases the probability of selecting only degenerate eigenvalues. Therefore, by considering a combined set $\left\{\underline{\boldsymbol{\rho}}_{z z}^{\mathbf{a}}\left(t_{i}, f_{i}\right) \mid i=1, \ldots, p\right\}$ one can improve the robustness of the joint diagonalization procedure. Note that if two source signals have identical $(t, f)$ signatures, it is expected intuitively that they cannot be separated even if one includes all information available in the $(t, f)$ domain.

The joint diagonalization of a set $\left\{\mathbf{M}_{k} \mid k=1, \ldots, p\right\}$ of $p$ matrices is formulated as the maximization of the following cost function [3]:

$$
C(\mathbf{V}) \triangleq \sum_{k=1}^{p} \sum_{i=1}^{n}\left|\mathbf{v}_{i}^{H} \mathbf{M}_{k} \mathbf{v}_{i}\right|^{2}
$$

over the set of unitary matrices $\mathbf{V}=\left[\mathbf{v}_{1}, \ldots, \mathbf{v}_{n}\right]$ [3]. One way to get an efficient

\footnotetext{
${ }^{7}$ Given that the off-diagonal elements of $\boldsymbol{\rho}_{s s}^{\mathbf{a}}(t, f)$ are actually cross-terms, the source TFD matrix is quasi-diagonal for the $(t, f)$ points that correspond to a true component power concentration, i.e. a source auto-term.
} 
joint approximate diagonalization algorithm is to generalize the Jacobi technique [38] for the exact diagonalization of a single normal matrix [3].

\subsubsection{Source separation by joint off-diagonalization (JOD)}

The effect of selecting cross-term $(t, f)$ points is that the whitened MTFD matrices formulation becomes:

$$
\underline{\boldsymbol{\rho}}_{z z}^{\mathbf{c}}(t, f)=\mathbf{U} \boldsymbol{\rho}_{s s}^{\mathbf{c}}(t, f) \mathbf{U}^{H}
$$

where $\boldsymbol{\rho}_{s s}^{\mathbf{c}}(t, f)$ is off-diagonal. As the diagonal of $\boldsymbol{\rho}_{s s}^{\mathbf{c}}(t, f)$ are formed by elements that are auto-terms, the source TFD matrix is then quasi off-diagonal (i.e., diagonal entries are negligible i.e. $\simeq 0$ ) for each $(t, f)$ point that corresponds to a cross-term. The required unitary matrix $\mathbf{U}$ is estimated by joint off-diagonalization (JOD) of a combined set $\left\{\underline{\boldsymbol{\rho}}_{z z}^{\mathbf{c}}\left(t_{i}, f_{i}\right) \mid i=1, \ldots, q\right\}$ of $q$ source cross-term MTFD matrices [3].

Such JOD procedure is justified by realizing that the off-diagonalization of a single $n \times n$ matrix $\mathbf{N}$ means maximizing

$$
C(\mathbf{N}, \mathbf{V}) \triangleq-\sum_{i=1}^{n}\left|\mathbf{v}_{i}^{H} \mathbf{N} \mathbf{v}_{i}\right|^{2}
$$

over the set of unitary matrices $\mathbf{V}=\left[\mathbf{v}_{1}, \ldots, \mathbf{v}_{n}\right]$. This is because the Frobenius norm of a matrix is constant under unitary transform, i.e., $\|\mathbf{N}\|_{F}=\left\|\mathbf{V}^{H} \mathbf{N V}\right\|_{F}$. Hence, the JOD of a set $\left\{\mathbf{N}_{k} \mid k=1, \ldots, q\right\}$ of $n \times n$ matrices is formulated as the maximization of the JOD cost function:

$$
C(\mathbf{V}) \triangleq \sum_{k=1}^{q} C\left(\mathbf{N}_{k}, \mathbf{V}\right)=-\sum_{k=1}^{q} \sum_{i=1}^{n}\left|\mathbf{v}_{i}^{H} \mathbf{N}_{k} \mathbf{v}_{i}\right|^{2}
$$

under the same unitary constraint.

Then, in order to improve the robustness of separation procedure and take advantage of both auto-terms and cross-terms, one can combine joint diagonalization and joint off-diagonalization of two sets $\left\{\mathbf{M}_{k} \mid k=1, \ldots, p\right\}$ and $\left\{\mathbf{N}_{k} \mid k=1, \ldots, q\right\}$ of $n \times n$ matrices by maximizing the JD/JOD cost function:

$$
C(\mathbf{V}) \triangleq \sum_{i=1}^{n}\left(\sum_{k=1}^{p}\left|\mathbf{v}_{i}^{H} \mathbf{M}_{k} \mathbf{v}_{i}\right|^{2}-\sum_{k=1}^{q}\left|\mathbf{v}_{i}^{H} \mathbf{N}_{k} \mathbf{v}_{i}\right|^{2}\right)
$$


over the set of unitary matrices $\mathbf{V}=\left[\mathbf{v}_{1}, \ldots, \mathbf{v}_{n}\right]$. Then, the combined JD/JOD criterion can be applied to a combined set of $p$ (source auto-term) MTFD matrices and $q$ (source cross-term) MTFD matrices in order to estimate the unitary matrix $\mathbf{U}$.

\section{Notes:}

(1) The performance of the JD or JOD of MTFD matrices in retrieving the unitary matrix $\mathbf{U}$ depends strongly on correctly selecting auto-term and crossterm points [3]. Therefore, it is critical to define a selection method that can discriminate between auto-term and cross-term points based only on the MTFD observations matrices. One possible solution is to exploit the off-diagonal structure of the source cross-term MTFD matrices and the invariance of Trace operation under a unitary transformation. More specifically, for a source cross-term MTFD matrix, we have

$$
\operatorname{Trace}\left(\underline{\boldsymbol{\rho}}_{z z}^{\mathbf{c}}(t, f)\right)=\operatorname{Trace}\left(\mathbf{U} \boldsymbol{\rho}_{s s}^{\mathbf{c}}(t, f) \mathbf{U}^{H}\right)
$$

Then, knowing that the source cross-term MTFD matrices are off-diagonal (i.e. the diagonal elements are equal to zero), and that $\operatorname{Trace}\left(\mathbf{U} \mathbf{M} \mathbf{U}^{H}\right)=\operatorname{Trace}(\mathbf{M})$ if $\mathbf{U}$ is a unitary matrix, then:

$$
\operatorname{Trace}\left(\mathbf{U} \boldsymbol{\rho}_{s s}^{\mathbf{c}}(t, f) \mathbf{U}^{H}\right)=\operatorname{Trace}\left(\boldsymbol{\rho}_{s s}^{\mathbf{c}}(t, f)\right) \approx 0 .
$$

Based on this observation, the following testing procedure applies:

1) if $\frac{\operatorname{Trace}\left(\underline{\boldsymbol{\rho}}_{z z}(t, f)\right)}{\left\|\underline{\boldsymbol{\rho}}_{z z}(t, f)\right\|}<\epsilon, \longrightarrow$ then, allocate the $(t, f)$ point as cross-term;

2) if $\frac{\operatorname{Trace}\left(\underline{\boldsymbol{\rho}}_{z z}(t, f)\right)}{\left\|\underline{\boldsymbol{\rho}}_{z z}(t, f)\right\|}>\epsilon, \longrightarrow$ then, allocate the $(t, f)$ point as auto-term; where $\epsilon$ is a 'small' positive real scalar (typically, $\epsilon=0.05$ ) [3].

(2) In effect, the source cross-term MTFD matrices are not totally off-diagonal, given that some auto-terms main lobes or side lobes overlap with the areas where cross-terms are dominant. This is like the case of joint diagonalization of MTFD matrices selecting auto-term points [3], where the source auto-term MTFD matrices are not totally diagonal because of cross-term overlap. This 
weakness is compensated by the joint approximation and robustness properties of the JD/JOD algorithm.

(3) The above results suggest that other classes of TFDs and related methods may also benefit from BSS; e.g. a cumulant-based $4^{\text {th }}$ order WVD or timevarying trispectrum can be utilized for source separation [39]. Blind separation of more sources than sensors (underdetermined BSS) is solved using a $(t, f)$ disjoint concept [40] (see Section 3.3).

Implementation details and the corresponding MatLAB code of the above algorithm are described in [5].

\subsubsection{Experiment: Separation of Instantaneous Mixtures}

In this example, three synthetic signals are generated with sampling frequency $1 \mathrm{~Hz}$ such that:

$$
\begin{aligned}
& s_{1}(t)=\exp \left(-j 2 \pi \frac{0.5}{256} t^{2}\right), \\
& s_{2}(t)=\exp \left(-j 2 \pi\left(0.5 t-\frac{0.5}{256} t^{2}\right)\right), \\
& s_{3}(t)=\exp (-j 2 \pi(0.3 t)),
\end{aligned}
$$

as depicted in the first row of Fig. 11. The generated signals are mixed using an instantaneous noisy uniform linear array model, to be received on $m=6$ sensors with an SNR of $30 \mathrm{~dB}$ (Fig. 12). The received mixtures are whitened, and their MTFD using WVD is computed for the selection of auto and cross-terms. Finally, the un-mixing matrix is estimated, using a joint diagonalization/joint off-diagonalization algorithm, and estimated sources are classified using their time-frequency correlation with the original signals, as depicted in the second row of Fig. 11. The results presented in this section can be reproduced using the codes provided in [5].

\subsection{BSS of convolutive mixtures based on MTFDs}

\subsubsection{Signal Model in the convolutive mixture case}

The convolutive case involves delayed elements caused e.g. by a multi-path propagation. The multiple input multiple output (MIMO) linear time invariant 

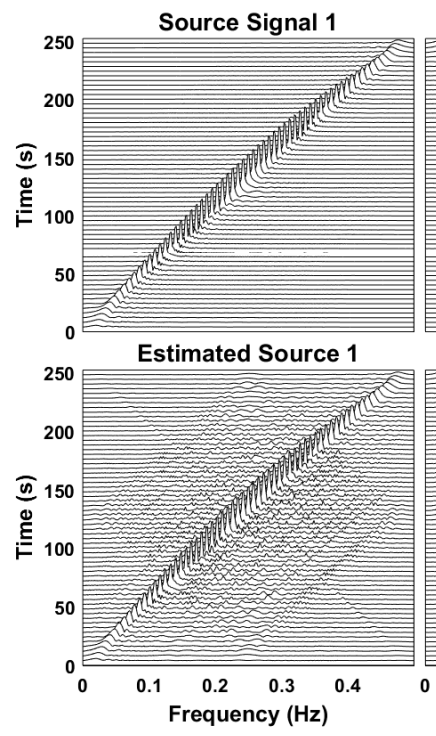

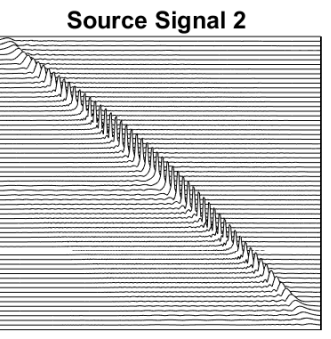

Estimated Source 2

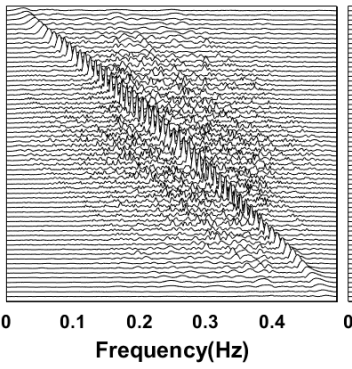

Source Signal 3

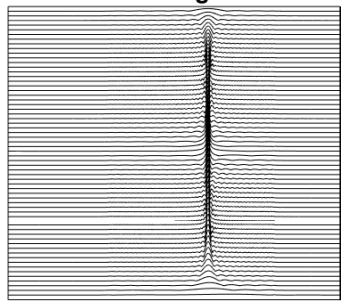

Estimated Source 3

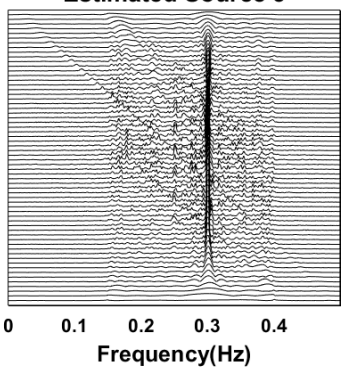

Figure 11: Blind source separation of three source signals $(n=3)$ based on MTFDs.

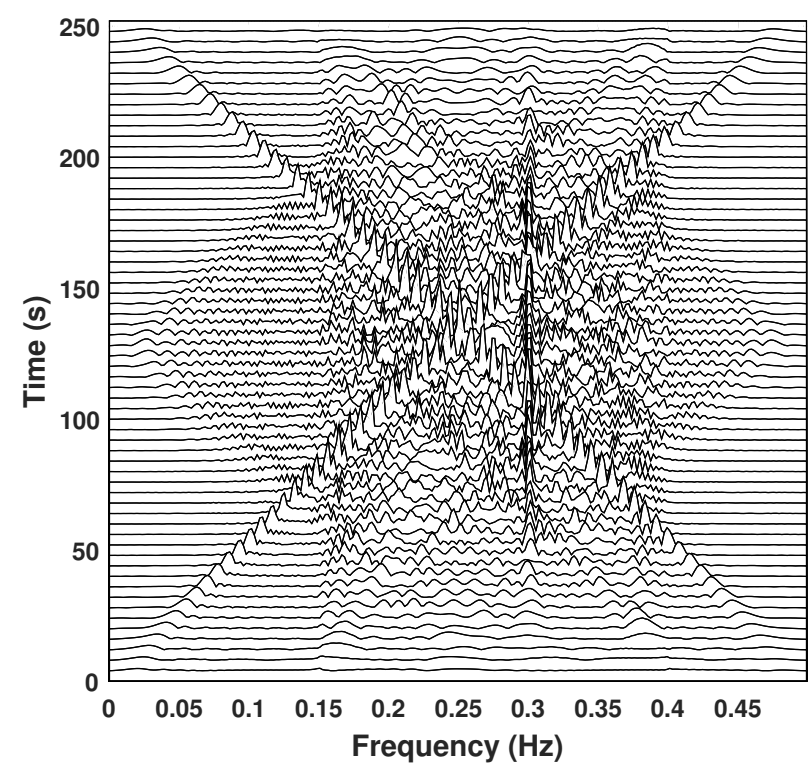

Figure 12: Received mixed signal: Received Signal on Sensor 3 with $S N R=30 d B$.

signal model can then be expressed as $\mathbf{z}(t)=\sum_{k=0}^{K} \mathbf{A}(k) \mathbf{s}(t-k)+\boldsymbol{\eta}(t)$ i.e. 
Eq.(15). As discussed earlier, two assumptions are made; (1) that the sources have different sparse $(t, f)$ signatures and $(2)$ the channel matrix $\tilde{\mathbf{A}}$ formulated in (40) is full column rank; this means that all the filters $\left\{a_{i j}(k)\right\}_{k=0}^{K}$ are stable [41]. Eq. (15) can be rewritten in matrix form as:

$$
\tilde{\mathbf{z}}(t)=\tilde{\mathbf{A}} \tilde{\mathbf{s}}(t)+\tilde{\boldsymbol{\eta}}(t),
$$

where $\tilde{\mathbf{s}}(t), \tilde{\mathbf{z}}(t), \tilde{\boldsymbol{\eta}}(t)$ and $\tilde{\mathbf{A}}$ are further described below:

$$
\begin{aligned}
\tilde{\mathbf{s}}(t) & =\left[s_{1}(t), \ldots, s_{1}\left(t-\left(K+K^{\prime}\right)+1\right), \ldots, s_{n}\left(t-\left(K+K^{\prime}\right)+1\right)\right]^{T}, \\
\tilde{\mathbf{z}}(t) & =\left[z_{1}(t), \ldots, z_{1}\left(t-K^{\prime}+1\right), \ldots, z_{m}\left(t-K^{\prime}+1\right)\right]^{T}, \\
\tilde{\boldsymbol{\eta}}(t) & =\left[\eta_{1}(t), \ldots, \eta_{1}\left(t-K^{\prime}+1\right), \ldots, \eta_{m}\left(t-K^{\prime}+1\right)\right]^{T},
\end{aligned}
$$

$$
\tilde{\mathbf{A}}=\left[\begin{array}{ccc}
\mathbf{A}_{11} & \cdots & \mathbf{A}_{1 n} \\
\vdots & \ddots & \vdots \\
\mathbf{A}_{m 1} & \cdots & \mathbf{A}_{m n}
\end{array}\right]
$$

with

$$
\mathbf{A}_{i j}=\left[\begin{array}{ccccc}
a_{i j}(0) & \cdots & a_{i j}(K) & \cdots & 0 \\
& \ddots & \ddots & \ddots & \\
0 & \cdots & a_{i j}(0) & \cdots & a_{i j}(K)
\end{array}\right],
$$

where $\tilde{\mathbf{A}}$ is an $m K^{\prime} \times n\left(K+K^{\prime}\right)$ matrix and $\mathbf{A}_{i j}$ are $K^{\prime} \times\left(K+K^{\prime}\right)$ matrices. The parameter $K^{\prime}$ is a slide window size chosen such that $m K^{\prime} \geq n\left(K+K^{\prime}\right)$ to ensure that the matrix $\tilde{\mathbf{A}}$ is invertible.

The formalism is similar to the instantaneous mixture case. The data MTFD matrices still have the same expression as in Eq. (21). But the source autoterm matrices $\boldsymbol{\rho}_{\tilde{s} \tilde{s}}(t, f)$ are no longer diagonal, but block-diagonal ${ }^{8}$ where each diagonal block is of size $\left(K+K^{\prime}\right) \times\left(K+K^{\prime}\right)$. Similarly, the source cross-term matrices are no longer off-diagonal but block off-diagonal. This block-diagonal

\footnotetext{
${ }^{8}$ The block diagonal characteristic comes from the property that cross-terms between $s_{i}(t)$ and $s_{i}(t-d)$ are not zero as they relate to the local correlation structure of the signal.
} 
or block off-diagonal property enables BSS to work in this case; as discussed in the next section.

\subsubsection{BSS using MTFD matrices for convolutive mixtures}

Let us now generalize the BSS method for the instantaneous case presented in Section 3.1 to the case of convolutive mixtures.

i) Data whitening preprocessing.

For BSS of instantaneous mixtures, this approach constrains the mixing matrix $\tilde{\mathbf{A}}$ to be orthogonal, which is not the case in general. To match assumptions with reality, the first step of the procedure is to then whiten the data vector $\tilde{\mathbf{z}}(t)$ to fulfill the orthogonality constraint. This is done by processing $\tilde{\mathbf{z}}(t)$ with a whitening matrix $\mathbf{H}$, which is an $n\left(K^{\prime}+K\right) \times m K^{\prime}$ matrix verifying:

$$
\mathbf{H} \mathbf{R}_{\tilde{z} \tilde{z}} \mathbf{H}^{H}=\left(\mathbf{H} \tilde{\mathbf{A}} \mathbf{R}_{\tilde{s} \tilde{s}}^{\frac{1}{2}}\right)\left(\mathbf{H} \tilde{\mathbf{A}} \mathbf{R}_{\tilde{s} \tilde{s}}^{\frac{1}{2}}\right)^{H}=\mathbf{I}_{n\left(K^{\prime}+K\right)},
$$

where $\mathbf{R}_{\tilde{z} \tilde{z}}$ and $\mathbf{R}_{\tilde{s} \tilde{s}}$ denote the covariance matrices of $\tilde{\mathbf{z}}(t)$ and $\tilde{\mathbf{s}}(t)$, respectively.

Eq. (42) shows that if $\mathbf{H}$ is a whitening matrix and if $\mathbf{R}_{\tilde{s} \tilde{s}}^{\frac{1}{2}}$ (Hermitian square root matrix of $\mathbf{R}_{\tilde{s} \tilde{s}}$ ) is block diagonal, then the following matrix

$$
\mathbf{U}=\mathbf{H} \tilde{\mathbf{A}} \mathbf{R}_{\tilde{s} \tilde{s}}^{\frac{1}{2}}
$$

is an $n\left(K^{\prime}+K\right) \times n\left(K^{\prime}+K\right)$ unitary matrix. The whitening matrix $\mathbf{H}$ can be determined from the eigendecomposition of the data covariance matrix $\mathbf{R}_{\tilde{z} \tilde{z}}$ as its inverse square root [3].

ii) Separation using matrix joint block diagonalization.

Recall the whitened MTFD matrices $\underline{\boldsymbol{\rho}}_{\tilde{z} \tilde{z}}(t, f)=\mathbf{H} \boldsymbol{\rho}_{\tilde{z} \tilde{z}}(t, f) \mathbf{H}^{H}$ as defined in (31). Combining Eq.(39) and Eq.(43) leads to:

$$
\underline{\boldsymbol{\rho}}_{\tilde{z} \tilde{z}}(t, f)=\mathbf{U} \mathbf{R}_{\tilde{s} \tilde{s}}^{-\frac{1}{2}} \boldsymbol{\rho}_{\tilde{s} \tilde{s}}(t, f) \mathbf{R}_{\tilde{s} \tilde{s}}^{-\frac{1}{2}} \mathbf{U}^{H}
$$

Let us denote $\boldsymbol{\rho}(t, f)=\mathbf{R}_{\tilde{s} \tilde{s}}^{-\frac{1}{2}} \boldsymbol{\rho}_{\tilde{s} \tilde{s}}(t, f) \mathbf{R}_{\tilde{s} \tilde{s}}^{-\frac{1}{2}}$. This then results in

$$
\underline{\boldsymbol{\rho}}_{\tilde{z} \tilde{z}}(t, f)=\mathbf{U} \boldsymbol{\rho}(t, f) \mathbf{U}^{H}
$$


As $\boldsymbol{\rho}(t, f)$ is block diagonal and the matrix $\mathbf{U}$ is unitary, the following property holds: any whitened MTFD matrix is then block diagonal on the basis formed by the column vectors of matrix $\mathbf{U}$. As a consequence, the unitary matrix $\mathbf{U}$ can be determined by estimating the block diagonalization of the matrix $\underline{\boldsymbol{\rho}}_{\tilde{z} \tilde{z}}(t, f)$. As for the joint diagonalization approach presented in Section 3.1.2, one can use the joint block diagonalization of a set $\left\{\underline{\boldsymbol{\rho}}_{\tilde{z} \tilde{z}}\left(t_{i}, f_{i}\right) ; i=1, \ldots, p\right\}$ of $p$ whitened MTFD matrices in order to reduces the probability of selecting only degenerate eigenvalues, and then improve the robustness of the joint block-diagonalization. A similar procedure can be used with the joint block off-diagonalization of the source cross-term MTFD matrices.

This joint block-diagonalization (JBD) is obtained by maximizing the following criterion under unitary transform:

$$
C(\mathbf{U}) \triangleq \sum_{k=1}^{p} \sum_{l=1}^{n} \sum_{i, j=\left(K^{\prime}+K\right)(l-1)+1}^{\left(K^{\prime}+K\right) l}\left|\mathbf{u}_{i}^{*} \underline{\boldsymbol{\rho}}_{\tilde{z} \tilde{z}}\left(t_{k}, f_{k}\right) \mathbf{u}_{j}\right|^{2}
$$

over the set of unitary matrices $\mathbf{U}=\left[\mathbf{u}_{1}, \ldots, \mathbf{u}_{n\left(K^{\prime}+K\right)}\right]$. To perform the above, one can use an efficient Jacobi-like algorithm for joint block diagonalization algorithm such as [38, 42].

After the unitary matrix $\mathbf{U}$ is retrieved (up to a block diagonal unitary matrix $\mathbf{P}$ due to the inherent JBD problem indeterminacy [43]), the estimated signals are then estimated up to a filter by:

$$
\widehat{\widetilde{\mathbf{s}}}(t)=\mathbf{U}^{H} \mathbf{H} \tilde{\mathbf{z}}(t)
$$

By "up to a filter" we mean that the separated sources correspond to filtered versions of the original ones, i.e. $\widehat{s}_{i}(t)=s_{i}(t) * h_{i}(t)$ where $h_{i}(t)$ is an unknown filter and $*$ stands for the convolution. According to (39) and (43), the estimated signals verify,

$$
\widehat{\widetilde{\mathbf{s}}}(t)=\mathbf{P} \mathbf{R}_{\tilde{s} \tilde{s}}^{-\frac{1}{2}} \widetilde{\mathbf{s}}(t)
$$

where, the matrix $\mathbf{R}_{\tilde{s} \tilde{s}}^{-\frac{1}{2}}$ is block diagonal and $\mathbf{P}$ is a block diagonal unitary matrix. 
iii) Assumptions and reality.

The performance of the above algorithms may be affected by a few points.

(1) In practice, it is sufficient that only $n$ signals among the $n\left(K^{\prime}+K\right)$ recovered ones are selected. One chooses the signals resulting in the smallest cross-terms coefficients. This result is obtained as a byproduct of the joint block diagonalization procedure with no additional required computations.

(2) The algorithm yields a source separation up to a filter, instead of the full MIMO deconvolution procedure. An alternative is to apply a SIMO (Single Input Multiple Output) deconvolution/equalization [44] to the separated sources.

\subsubsection{Experiment: Separation of Convolutive Mixtures}

In this experiment, two synthetic signals $(n=2)$ are generated with sampling frequency $1 \mathrm{~Hz}$ as:

$$
\begin{aligned}
& s_{1}(t)=\exp \left(-j 2 \pi\left(0.5 t-\frac{0.5}{256} t^{2}\right)\right), \\
& s_{2}(t)=\exp \left(-j 2 \pi 10^{-5} t^{3}\right)
\end{aligned}
$$

as depicted in the first row of Fig. 13. The generated signals are passed through a noisy convolutive invariant filter with length $K=1$ representing the mixing model and then received on four sensors $(m=4)$ with $40 \mathrm{~dB}$ SNR (Fig. 14). The received mixtures are whitened, and their MTFD is computed using WVD for the selection of auto-terms. The estimated sources are obtained by applying a separation matrix given by the joint block diagonalization of selected autoterms with window size $K^{\prime}=2$. Estimated filtered sources are then classified using their TF correlation with the original signals, as depicted in the last three rows of Fig. 13.

In the second experiment, a pair of one seconds soundtracks $(n=2)$, car start-up and seagull sounds, are used as illustrated in the first row of Figs. 15 and 16. Other one seconds soundtracks can be obtained from [5], while their original full length can be downloaded from [45]. The used sounds are passed through a noisy convolutive invariant filter, describing the mixing model, to be received on three sensors $(m=3)$, as shown in Fig. 17. The received mixtures 


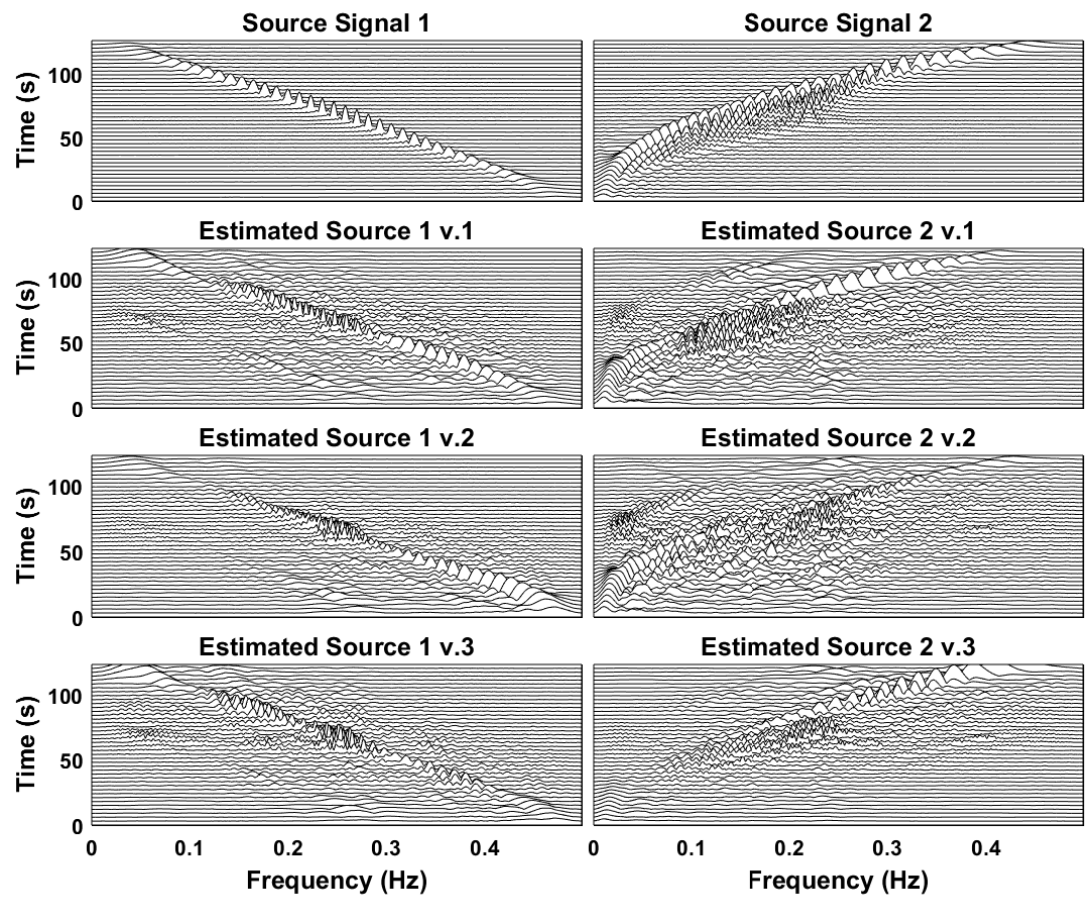

Figure 13: Blind source separation of two source signals based on MTFDs.

are then whitened and their MTFD is computed using WVD for the selection of auto-terms. Estimated filtered sources are classified using their time-frequency correlation with the original signals, as depicted in the last three rows of Figs. 15 and 16.

The above results can be reproduced using the codes provided in [5].

\subsection{Under-determined blind source separation (UBSS)}

In applications such as telecommunications or geophysics, the received signals form a mixture. The user is not necessarily interested in the whole signal mixture, but rather in one or more particular signal components. For example, in wireless communications the received signal is often a mixture of several source signals (multiple access interference) but the user may be interested in recovering only one or a few source signals. In the case where the signal com- 


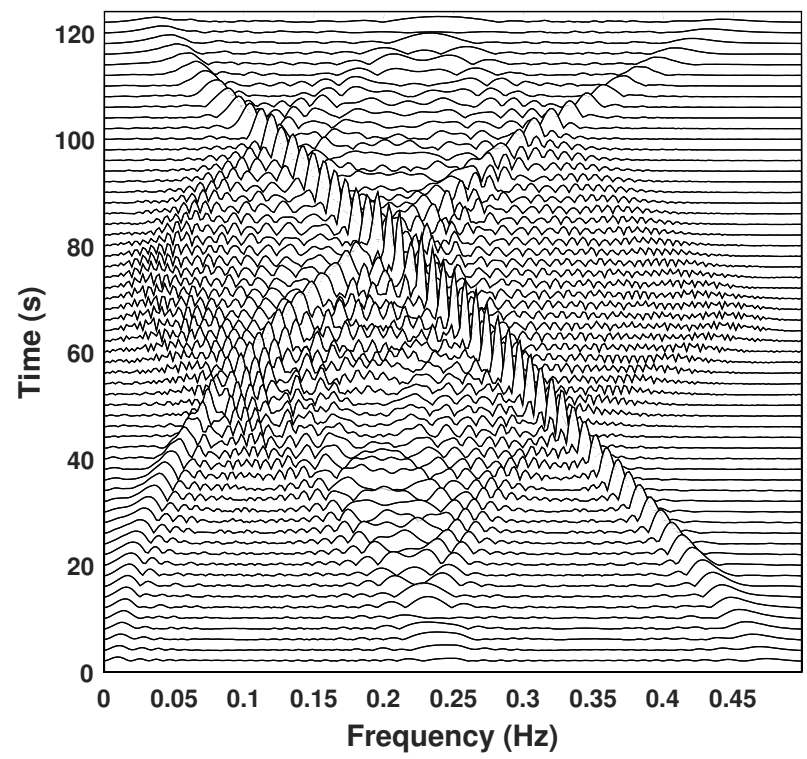

Figure 14: Received mixed signal (received signal on sensor 2 with $S N R=40 d B$ ).

ponent are non-stationary one can use MTFDs to extract the desired source signal, and separate/recover any signal component.

The BSS algorithms given in Section 3.1 have apparent limitations in situations where there are more sources than sensors with a failure to separate sources. The focus of this section is to address this problem known as the underdetermined BSS (UBSS).

This section therefore assumes that (1) there are more sources than sensors, and (2) the sources are non-stationary FM signals as encountered in wireless communications and geophysics.

\subsubsection{Data model and assumptions}

As in Section 3.1, let us assume that an $n$-dimensional vector $\mathbf{s}(t)=$ $\left[s_{1}(t), \ldots, s_{n}(t)\right]^{T}$ represents $n$ non-stationary source signals denoted $s_{i}(t)$, $i=1, \ldots, n$. These signals propagate through a medium and arrive at an array of $m$ sensors which records a set of mixed signals described by an $m$-dimensional vector $\mathbf{z}(t)=\left[z_{1}(t), \ldots, z_{m}(t)\right]^{T}$. This situation is described by the data model presented in Section 2.2.1 Eq.(12) such that $\mathbf{z}(t)=\mathbf{A} \mathbf{s}(t)+\boldsymbol{\eta}(t)$. 


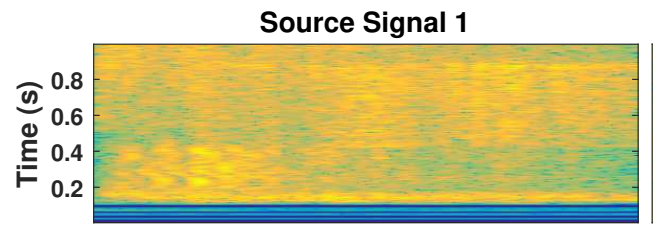

Estimated Source 1 v.1

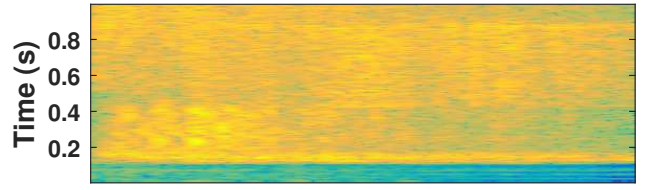

Estimated Source 1 v.2

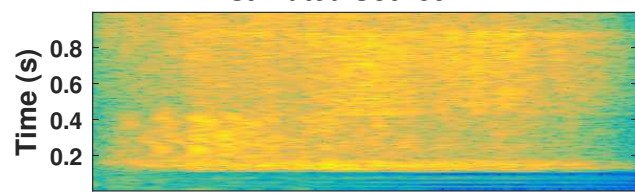

Estimated Source 1 v.3

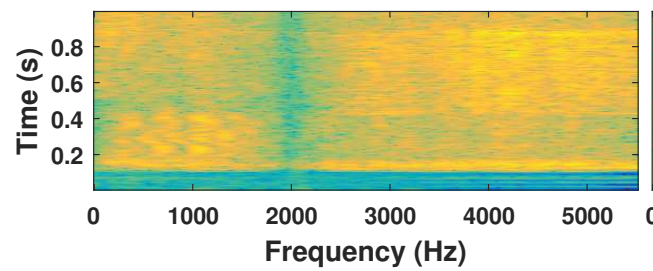

Source Signal 2

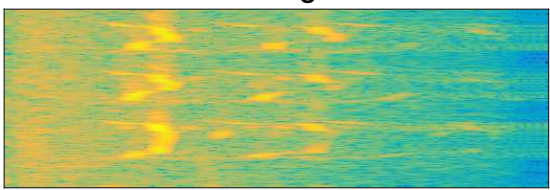

Estimated Source 2 v.1

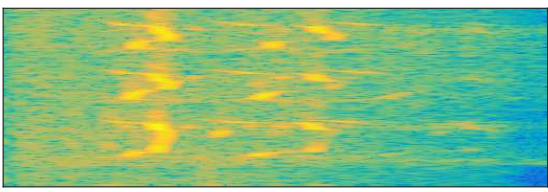

Estimated Source 2 v.2

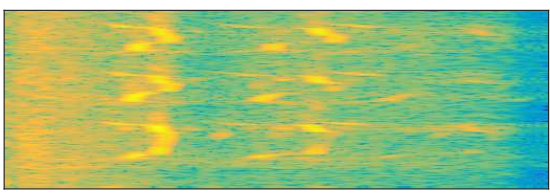

Estimated Source 2 v.3

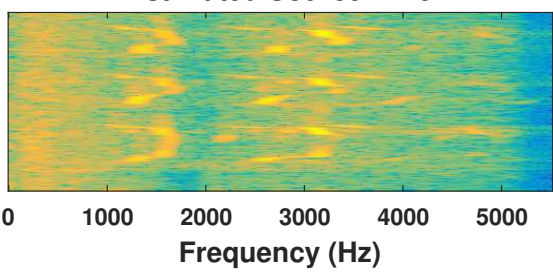

Figure 15: Blind source separation of two audio source signals based on MTFDs (timefrequency representation).

In the case where the number of sensors $m$ is less than the number of sources $n$, i.e. UBSS problem, the mixing matrix $\mathbf{A}$ is not (left) invertible. However, the column vectors of mixing matrix given by $\mathbf{A}=\left[\mathbf{a}_{1}, \mathbf{a}_{2}, \ldots, \mathbf{a}_{n}\right]$ are assumed to be pairwise linearly independent, i.e., for any $i, j \in 1,2, \ldots, n$ and $i \neq j, \mathbf{a}_{i}$ and $\mathbf{a}_{j}$ are linearly independent. This hypothesis is equivalent to assuming that the direction of arrival of each of the $n$ signal sources is different. As mentioned earlier, the sources are assumed to be multicomponent FM signals. This signal model finds application in speech analysis/synthesis [46]. In this case, the $(t, f)$ domain representing the sources shows a distinct pattern with multiple ridges. The $k^{\text {th }}$ source may be expressed as,

$$
s_{k}(t)=\sum_{l=1}^{M_{k}} s_{k, l}(t),
$$




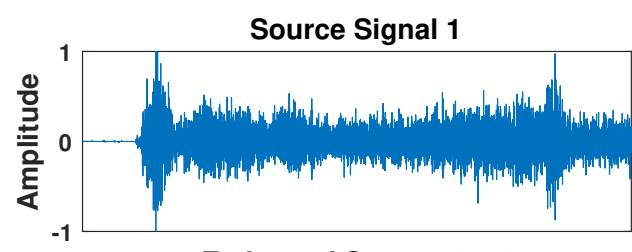

Estimated Source 1 v.1

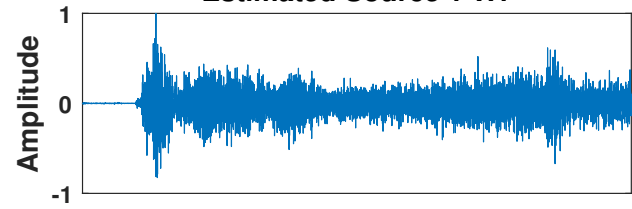

Estimated Source 1 v.2

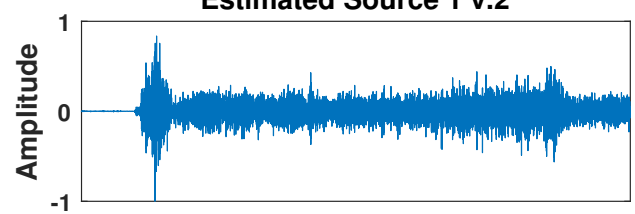

Estimated Source 1 v.3

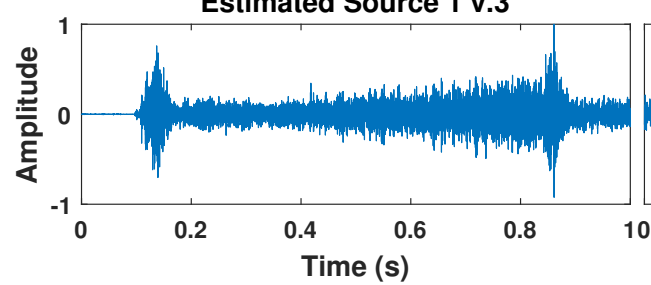

Source Signal 2

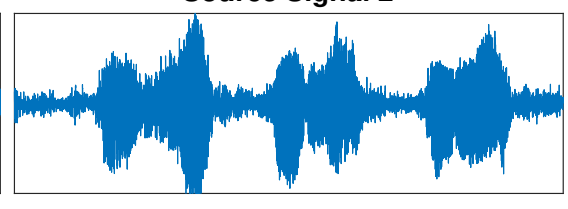

Estimated Source 2 v.1

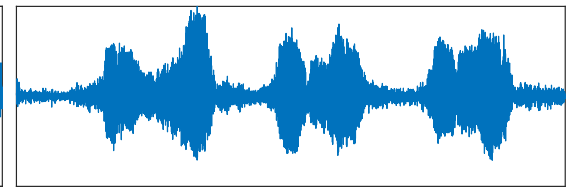

Estimated Source 2 v.2

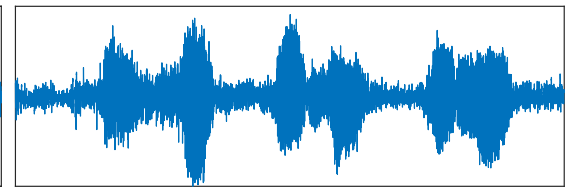

Estimated Source 2 v.3

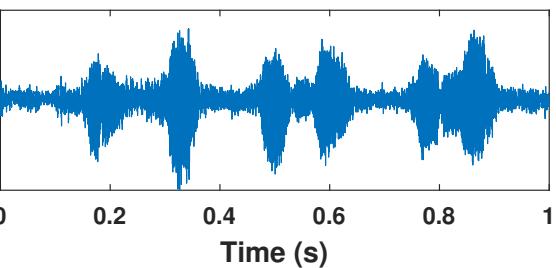

Figure 16: Blind source separation of two audio source signals based on MTFDs (time domain representation).

where each component $s_{k, l}(t)$, has an AM/FM form such as:

$$
s_{k, l}(t)=a_{k, l}(t) e^{j \phi_{k, l}(t)}
$$

It is assumed that $s_{k, l}(t)$ has only one ridge in the $(t, f)$ domain. Fig. 18 shows an example of a multicomponent signal, consisting of three components.

\subsubsection{UBSS using Vector Clustering}

The following presents a UBSS algorithm with a vector clustering approach using the assumption that the sources are disjoint in the $(t, f)$ domain [40].

i) Model assumptions.

This approach assumes that (1) the sources have different sparse $(t, f)$ signatures, $(2)$ the sources are consequently disjoint in the $(t, f)$ domain (Fig. 19) in 


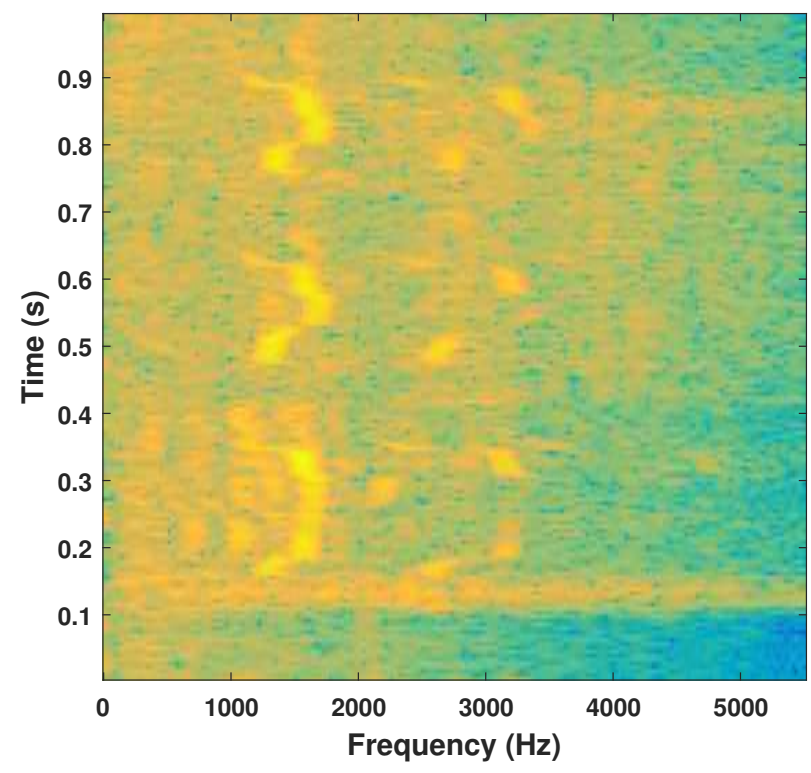

Figure 17: Received mixed signal (received signal on sensor 3): (time-frequency representation of the convolutive mixture of two audio sources).

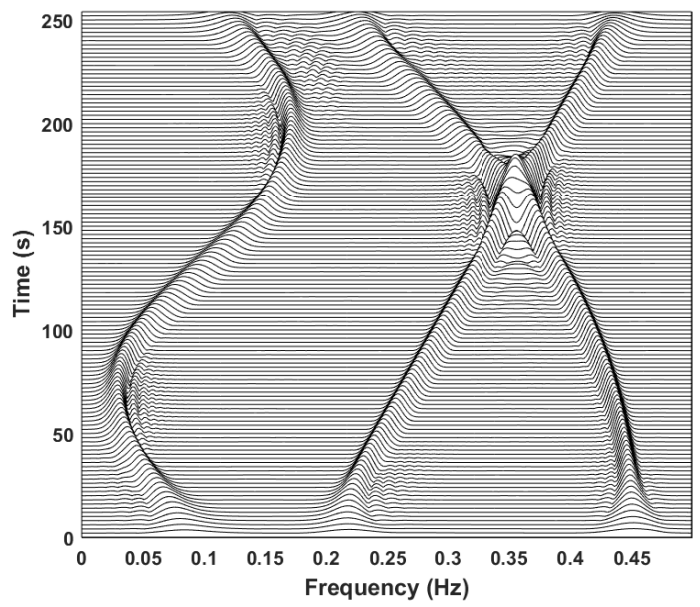

Figure 18: A time-frequency distribution of a multicomponent non-stationary signal: (Compact kernel distribution (CKD) with parameters $c=1, D=0.1$ and $E=0.1$ ).

the sense that their $(t, f)$ supports are disjoint. The $(t, f)$ support of a signal $s(t)$ is defined as $\left\{(t, f) \mid \rho_{s s}(t, f) \neq 0\right\}$ where $\rho_{s s}(t, f)$ is the TFD of $s(t)$. 


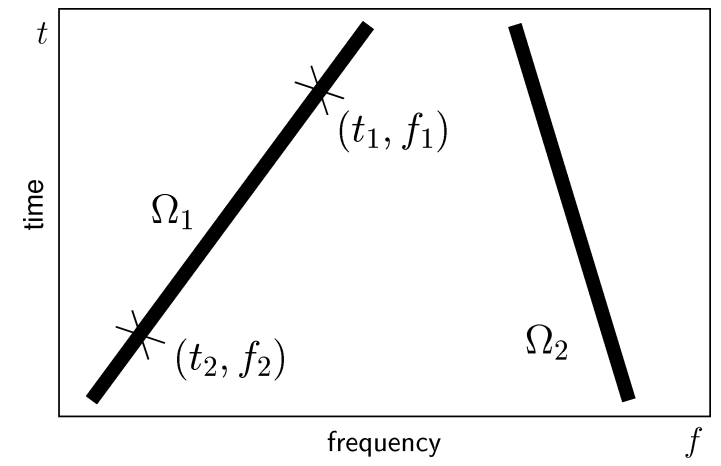

Figure 19: $(t, f)$ orthogonal sources; the $(t, f)$ supports of two sources are disjoint.

The properties of TFDs suggest that the $(t, f)$ disjoint assumption is too restrictive and cannot be verified exactly in practice. However, only an approximate disjoint assumption (quasi-disjoint) is required in practice to separate sources [40]; e.g. two linear FM signals with different gradients satisfy the quasi-disjoint assumption.

Under these assumptions, let us consider two auto-term MTFD matrices (as defined in Section 3.1) of the observation; then the MTFDs $\boldsymbol{\rho}_{z z}\left(t_{1}, f_{1}\right)$ and $\boldsymbol{\rho}_{z z}\left(t_{2}, f_{2}\right)$ corresponding to the same source $s_{i}(t)$ verify:

$$
\begin{aligned}
& \boldsymbol{\rho}_{z z}\left(t_{1}, f_{1}\right)=\rho_{s_{i} s_{i}}\left(t_{1}, f_{1}\right) \mathbf{a}_{i} \mathbf{a}_{i}^{H}, \\
& \boldsymbol{\rho}_{z z}\left(t_{2}, f_{2}\right)=\rho_{s_{i} s_{i}}\left(t_{2}, f_{2}\right) \mathbf{a}_{i} \mathbf{a}_{i}^{H} .
\end{aligned}
$$

Eq.(51) shows that $\boldsymbol{\rho}_{z z}\left(t_{1}, f_{1}\right)$ and $\boldsymbol{\rho}_{z z}\left(t_{2}, f_{2}\right)$ have the same principal eigenvector $\mathbf{a}_{i}$. One can then design an algorithm which groups together all auto-term points corresponding to the same principal eigenvector associated with a particular source signal. This source TFD $\left(\rho_{s_{i} s_{i}}(t, f)\right)$ is then estimated as the principal eigenvalue of the MTFD matrices at the auto-term points. Fig.20 illustrates the corresponding flowchart.

ii) A four stage design and implementation procedure.

The UBSS algorithm using vector clustering has four main stages: 


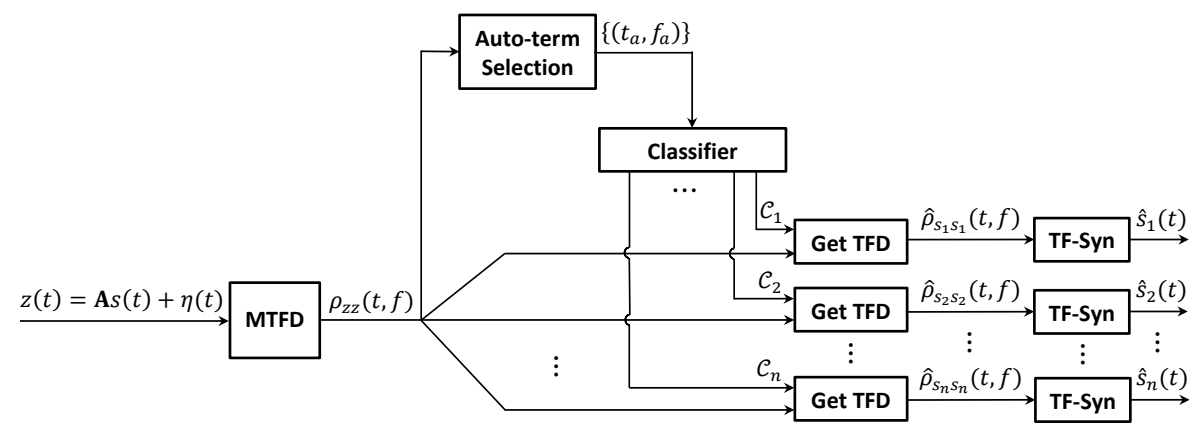

Figure 20: Diagram of the UBSS algorithm.

\section{(1) MTFD computation and noise thresholding}

These MTFD matrices process the received signal to extract the source signals. The computational cost is reduced by processing only "significant" MTFD matrices, using a noise thresholding applied to the signal TFD. A threshold $\epsilon_{1}$ is used to keep only the points $\left\{\left(t_{s}, f_{s}\right)\right\}$ with sufficient energy. Typically, $\epsilon_{1}=0.05$ of the point with maximum energy [40]. This step can be summarized as:

$$
\text { Keep }\left(t_{s}, f_{s}\right) \text { if }\left\|\boldsymbol{\rho}_{z z}\left(t_{s}, f_{s}\right)\right\|>\epsilon_{1} \text {. }
$$

\section{(2) Auto-term selection}

Separating the auto-term points from cross-term points requires an appropriate testing criterion. Given the sources TF disjoint requirement, each auto-term MTFD matrix is of rank one, or in practice it has one "large" eigenvalue relative to other eigenvalues. One can then use rank selection criteria, such as MDL (minimum description length) or AIC (Akaike information criterion) to discriminate $(t, f)$ points [47]. The result is then essentially to select auto-term points as those corresponding to MTFD matrices of rank one. This step can written formally as follows:

if $\left|\frac{\lambda_{\max }\left\{\boldsymbol{\rho}_{z z}(t, f)\right\}}{\operatorname{norm}\left\{\boldsymbol{\rho}_{z z}(t, f)\right\}}-1\right|>\epsilon_{2} \longrightarrow$ decide that $(t, f)$ is a cross-term point.

In the above, the parameter $\epsilon_{2}$ is a negligible positive scalar (typically, $\epsilon_{2}=10^{-4}$ $[40])$, and $\lambda_{\max }\left\{\boldsymbol{\rho}_{z z}(t, f)\right\}$ denotes the largest relative eigenvalue of $\boldsymbol{\rho}_{z z}(t, f)$. 


\section{(3) Clustering and source TFD estimation}

After selecting the auto-term points, one needs a clustering step for the spatial signatures of the sources. This step is done to separate (cluster) each $(t, f)$ point following its spatial component given by the corresponding principal eigenvector. The principle is that that two MTFD matrices associated with the same source signal have the same corresponding principal eigenvectors. Also, recall that such corresponding principal eigenvalues identify the desired source TFD. The above step can be implemented in 4 operations described below:

(a) For each auto-term point, $\left(t_{a}, f_{a}\right)$, find the principal eigenvector, $\mathbf{v}\left(t_{a}, f_{a}\right)$, and its associated eigenvalue, $\lambda\left(t_{a}, f_{a}\right)$, of $\boldsymbol{\rho}_{z z}\left(t_{a}, f_{a}\right)$.

(b) Given that the vectors $\left\{\mathbf{v}\left(t_{a}, f_{a}\right)\right\}$ are estimated up to a random phase multiplicative coefficient $e^{j \phi}, \phi \in[0,2 \pi)$, one can constraint, without loss of generality, their norms to be 1 and their first entries to be real positive.

(c) These vectors are then clustered into different classes $\left\{\mathcal{C}_{i} \mid i=1, \ldots, n\right\}$ by using any clustering algorithms such as the $k$-means algorithm [48].

(d) For each particular source $s_{i}$ (i.e. each class $\mathcal{C}_{i}$ ), calculate its TFD as:

$$
\hat{\rho}_{s_{i} s_{i}}(t, f)= \begin{cases}\lambda\left(t_{a}, f_{a}\right), & \text { if }(t, f)=\left(t_{a}, f_{a}\right) \in \mathcal{C}_{i} \\ 0, & \text { otherwise }\end{cases}
$$

Note that one can also estimate the mixing matrix $\mathbf{A}$ such that the column vectors of $\mathbf{A}$ are estimated as the centroid of each class $\mathcal{C}_{i}$ i.e.:

$$
\widehat{\mathbf{a}}_{i}=\frac{1}{\operatorname{Card}\left\{\mathcal{C}_{i}\right\}} \sum_{(t, f) \in \mathcal{C}_{i}} \mathbf{v}(t, f)
$$

where $\operatorname{Card}\left\{\mathcal{C}_{i}\right\}$ represents the number of elements in the set $\mathcal{C}_{i}$.

\section{(4) Source signal reconstruction}

The last stage is then to select an appropriate reconstruction technique to estimate the source signals, $s_{i}(t)(i=1, \ldots, n)$, from their corresponding TFD estimates $\hat{\rho}_{s_{i} s_{i}}$. The method in [49] can be exploited for TF synthesis from the 
WVD of separated sources, based on the following inversion property of the WVD [1, Chapter 3]:

$$
z(t)=\frac{1}{z(0)^{*}} \int_{\mathbb{R}} W_{z}\left(\frac{t}{2}, f\right) e^{j 2 \pi t f} d f
$$

In the next section, the TF-disjoint condition is relaxed by allowing the sources to be nondisjoint in the $(t, f)$ plane. This is to overcome the drawback of the BSS method presented above. Although this method worked well under the TF-almost-disjoint condition, it did not explicitly process the TF regions where the sources overlap. A point in the overlap area of two sources was assigned "by chance" to only one of the sources. As a result, the source that is allocated this point gets information about another source while the latter loses some of its own information. If the number of overlapping points increases (i.e., the TF-almost-disjoint condition is not met), the performance of the separation tends to reduce unless the overlapping points are processed correctly.

\subsubsection{UBSS for non-disjoint sources in the $(t, f)$ domain}

In most real life applications, the $(t, f)$ disjoint assumption is a restrictive condition that is not precisely and rigourously applicable. When this condition is not met, the separation performance degrades greatly at those overlapped $(t, f)$ points. In order to remedy this weakness, a subspace projection based separation method, with sparsity assumption relaxed, can be used $[4,50]$. In this algorithm, the $(t, f)$ representations of different sources are allowed to overlap, but only to the extent that there are less active sources at any $(t, f)$ point than the number of sensors. As this sparsity condition is more relaxed than the $(t, f)$ disjoint assumption so that the sources are allowed to overlap in the $(t, f)$ domain, it will be called TF nondisjoint.

Under the $(t, f)$ nondisjoint assumption, consider a particular $(t, f)$ point at which there are $\mathcal{K}$ sources $s_{\ell_{1}}(t), \ldots, s_{\ell_{\mathcal{K}}}(t)$ present, with $\mathcal{K}<m$ where $\ell_{1}, \ldots, \ell_{\mathcal{K}} \in\{1,2, \ldots, n\}$ denote the indexes of the sources present at the point $\left(t_{0}, f_{0}\right)$. The goal is to identify the sources that are present at the point $\left(t_{0}, f_{0}\right)$, i.e. $\ell_{1}, \ldots, \ell_{\mathcal{K}}$, and to estimate the TFD of each of these contributing sources. 
In order to model the received signal under this assumption, let us define the following sub-vector and sub-matrix:

$$
\begin{aligned}
\tilde{\mathbf{s}}(t) & =\left[s_{\ell_{1}}(t), \ldots, s_{\ell_{\mathcal{K}}}(t)\right]^{T}, \\
\tilde{\mathbf{A}}_{\ell} & =\left[\mathbf{a}_{\ell_{1}}, \ldots, \mathbf{a}_{\ell_{\mathcal{K}}}\right] .
\end{aligned}
$$

Then, under the $(t, f)$ nondisjoint assumption the instantaneous model reduces to the following

$$
\boldsymbol{\rho}_{z z}(t, f)=\tilde{\mathbf{A}}_{\ell} \boldsymbol{\rho}_{\tilde{s} \tilde{s}}(t, f) \tilde{\mathbf{A}}_{\ell}^{H} .
$$

Consequently, given that $\boldsymbol{\rho}_{\tilde{s} \tilde{s}}(t, f)$ is of full rank, we obtain

$$
\text { Range }\left\{\boldsymbol{\rho}_{z z}(t, f)\right\}=\text { Range }\left\{\tilde{\mathbf{A}}_{\ell}\right\}
$$

Let $\mathbf{Q}=\mathbf{I}_{m}-\mathbf{V} \mathbf{V}^{H}$ be the orthogonal projection matrix onto the noise subspace of $\boldsymbol{\rho}_{z z}(t, f)$, where $\mathbf{V}$ is the matrix formed by the $\mathcal{K}$ principal singular eigenvectors of $\boldsymbol{\rho}_{z z}(t, f)$. Then, from Eq. (58), we obtain:

$$
\left\{\begin{array}{l}
\mathbf{Q} \mathbf{a}_{i}=0, \quad i \in\left\{\ell_{1}, \ldots, \ell_{\mathcal{K}}\right\}, \\
\mathbf{Q} \mathbf{a}_{i} \neq 0, \quad \text { otherwise. }
\end{array} .\right.
$$

Assuming that the mixing matrix $\mathbf{A}$ has been estimated by methods such as the clustering based method presented in Section 3.3.2, the observation in Eq. (59) can be used to identify the indexes $\ell_{1}, \ldots, \ell_{\mathcal{K}}$ and therefore, the sources present at $\left(t_{0}, f_{0}\right)$. To implement this and account for the additive noise, one can detect these indexes by selecting the $\mathcal{K}$ smallest values from the set $\left\{\left\|\mathbf{Q} \mathbf{a}_{i}\right\| i=1, \ldots, n\right\}$, as mathematically expressed by:

$$
\left\{\ell_{1}, \ldots, \ell_{\mathcal{K}}\right\}=\arg \min _{i ; \mathcal{K}}\left\|\mathbf{Q} \mathbf{a}_{i}\right\|
$$

The MTFD values of the $\mathcal{K}$ sources at $\left(t_{0}, f_{0}\right)$ are calculated as the diagonal elements of the following matrix:

$$
\widehat{\boldsymbol{\rho}}_{\tilde{s} \tilde{s}}\left(t_{0}, f_{0}\right) \approx \tilde{\mathbf{A}}_{\ell}^{\#} \boldsymbol{\rho}_{z z}\left(t_{0}, f_{0}\right) \tilde{\mathbf{A}}_{\ell}^{\# H},
$$

where (\#) represents the Moore-Penrose's pseudo-inversion operator [51]. 


\subsubsection{Experiment: Underdetermined blind source separation}

In the first experiment, four TF disjoint source signals $(n=4)$ are generated with sampling frequency $1 \mathrm{~Hz}$ such that:

$$
\begin{aligned}
& s_{1}(t)=\exp \left(-j 2 \pi \frac{0.2}{256} t^{2}\right)+\exp \left(-j 2 \pi\left(0.2 t-\frac{0.2}{256} t^{2}\right)\right) \\
& s_{2}(t)=\exp (-j 2 \pi(0.25 t)) \\
& s_{3}(t)=\exp \left(-j 2 \pi\left(0.3 t+\frac{0.1}{256} t^{2}\right)\right) \\
& s_{4}(t)=\exp \left(-j 2 \pi\left(0.4 t+\frac{0.1}{256} t^{2}\right)\right)
\end{aligned}
$$

as depicted in the first row of Fig. 21. The generated signals are then mixed using an instantaneous noisy uniform linear array model, to be received on three sensors $(m=3)$ with an SNR of $30 \mathrm{~dB}$. The MTFDs of the received mixtures are computed and the separation is achieved by using a clustering method as depicted in the second row of Fig. 21.

In the second experiment, four non-disjoint source signals $(n=4)$ are generated using crossing LFMs. The first two signals are crossing LFMs interchangeably changing from $0.05 \mathrm{~Hz}$ to $0.2 \mathrm{~Hz}$, while the third and fourth signals are LFMs interchangeably changing from $0.3 \mathrm{~Hz}$ to $0.45 \mathrm{~Hz}$, as depicted in the first row of Fig. 22. The generated signals are then mixed using an instantaneous noisy uniform linear array model, to be received on three sensors $(m=3)$ with an SNR of $40 \mathrm{~dB}$ (Fig. 23). From Fig. 22, we can observe that the overlapping points are randomly allocated for one source, when utilizing the cluster-based algorithm. However, by using the subspace-based algorithm, the intersection points are redistributed to their corresponding two sources. In general, overlapping points in the non-disjoint case have been explicitly treated. This provides a visual performance comparison.

The above results can be reproduced using the codes provided in [5].

\subsection{Discussion}

The performance and limitations of BSS algorithms and their applications are further reviewed below. 

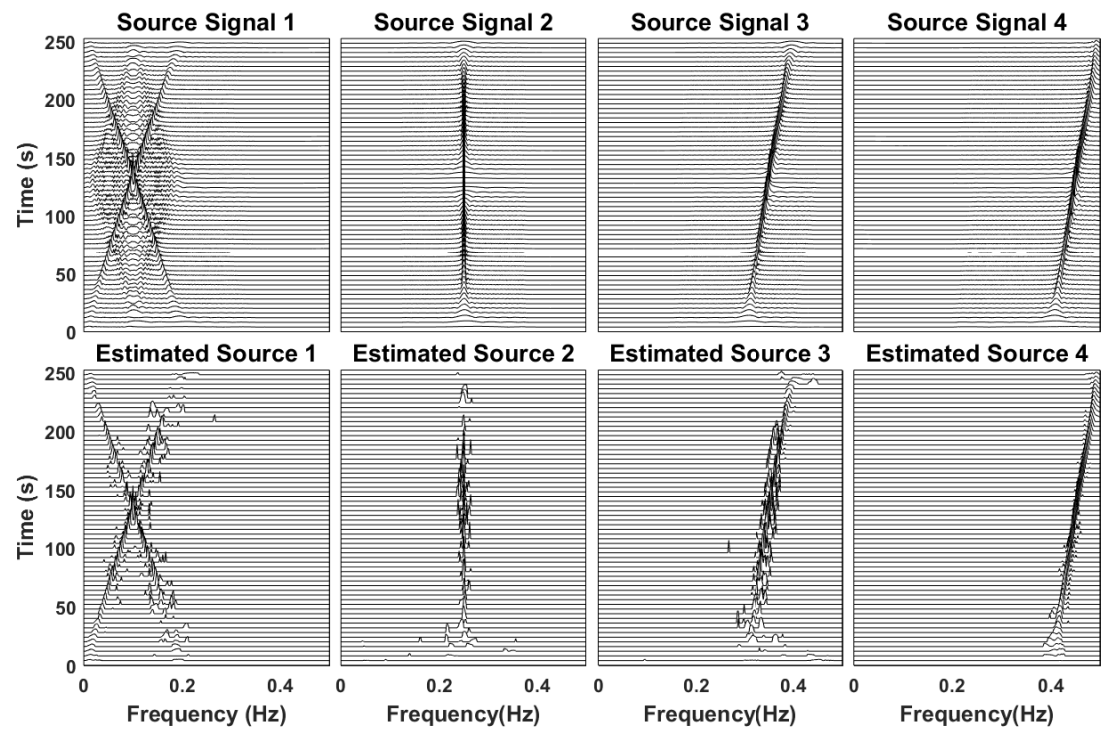

Figure 21: Underdetermined blind source separation of four source signals $(n=4)$ based on MTFDs received from an array of three sensors $(m=3)$ using clustering based method: (The first row represents the source signals, while the second row represents the estimated sources using UBSS clustering method).

\subsubsection{Auto-term selection}

In a noisy environment, the selection of auto-term TF points by the procedures presented in Sections 3.1 and 3.2 may become challenging when the signals are highly corrupted by noise. The spatial diversity, embedded in the MTFD matrix, can reduce noise and enhance the TF signatures of the signals of interest. This can be achieved by averaging the TFDs over all receiver sensors $[52]$.

\subsubsection{Number of sources for UBSS clustering step}

In the UBSS algorithm using vector clustering the number of sources $n$ is assumed known in the clustering step ( $k$-means). However, there exist other clustering methods that perform the class estimation as well as the estimation of the number $n$ [53]. Nevertheless, the results observed indicate that by using this kind of clustering method most of the time the number of classes is overes- 

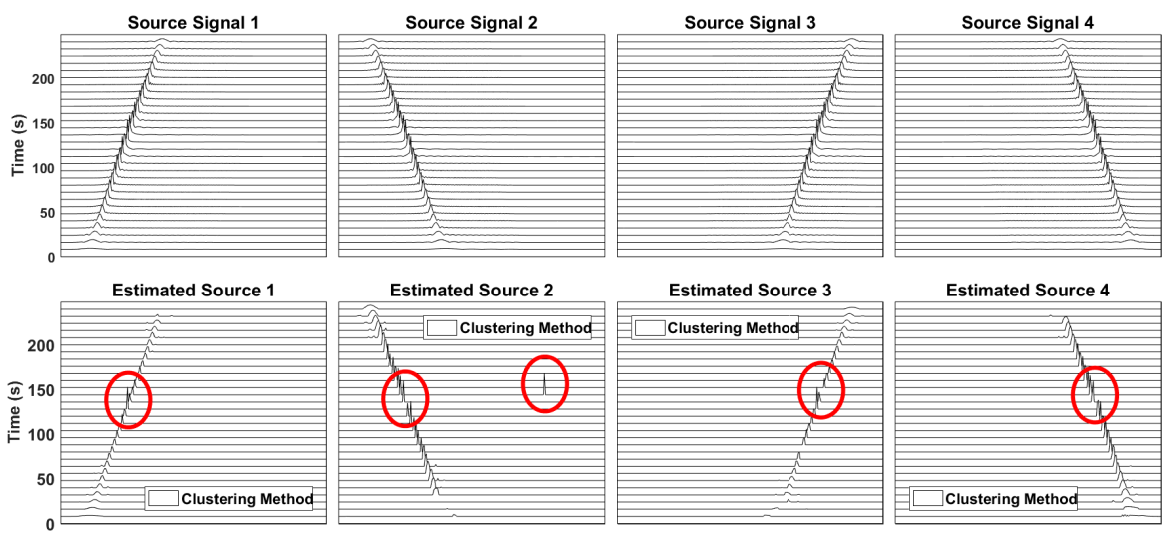

Estimated Source 2

Estimated Source 4
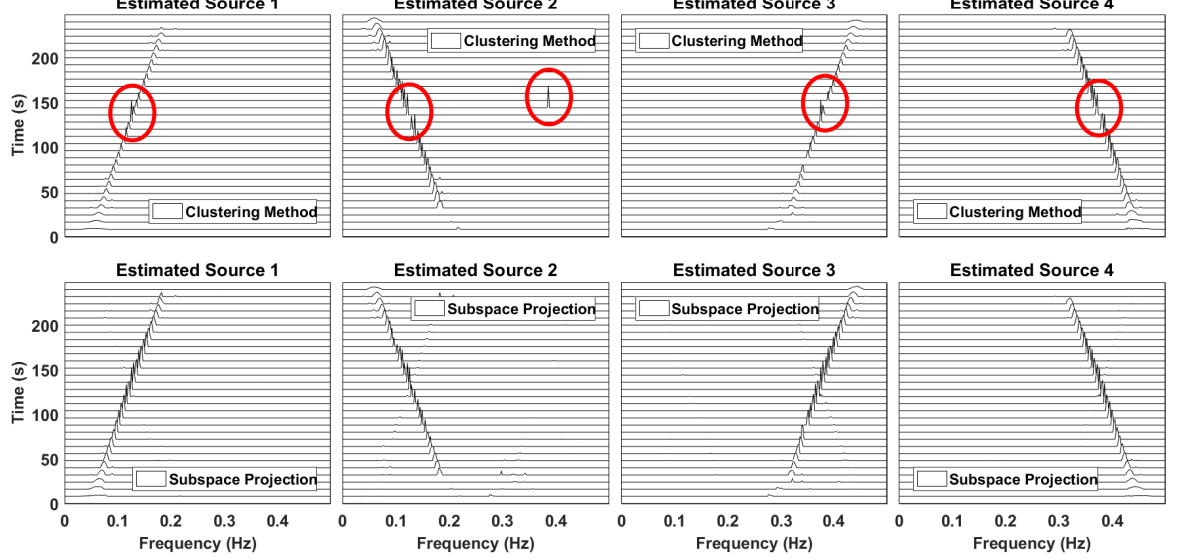

Estimated Source 4

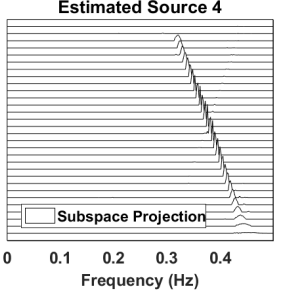

Figure 22: Underdetermined blind source separation of four non-disjoint source signals $(n=4)$ based on MTFDs received from an array of three sensors $(m=3)$ using clustering and subspace projection based methods: (The red circles highlight the overlaping point where the clustering methods fails to adequately estimate the sources).

timated, leading to poor source separation quality. Hence, robust estimation of the number of sources in the UBSS case remains a difficult open problem that deserves particular attention in future studies.

\subsubsection{Number of overlapping sources}

In the subspace based UBSS algorithm, one has to evaluate the number $\mathcal{K}$ of overlapping sources at a given $(t, f)$ point. This can be done by finding out the number of non-zero eigenvalues of $\boldsymbol{\rho}_{z z}(t, f)$ using criteria such as MDL or AIC [47]. It is also possible to consider a fixed (maximum) value of $\mathcal{K}$ that is used for all autosource $(t, f)$ points. Indeed, if the number of overlapping sources is less than $\mathcal{K}$, one would estimate close-to-zero source $(t, f)$ values. For example, 


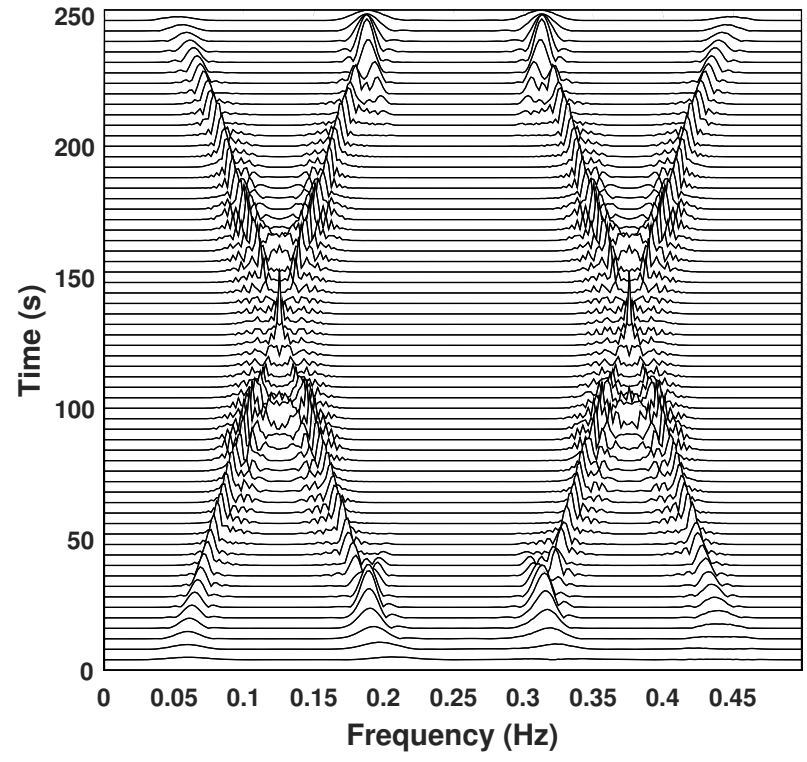

Figure 23: Received mixed signal (received signal on sensor 1 with $S N R=40 d B$ ).

if we assume $\mathcal{K}=2$ sources are present at a given $(t, f)$ point while only one source is effectively contributing, then one estimates one close-to-zero source $(t, f)$ values. This approach increases slightly the estimation error of the source signals (especially at low SNRs) but has the advantage of simplicity compared to using information theoretic-based criterion.

\subsubsection{Separation quality versus number of sources}

Although we are in the underdetermined case, the number of sources $n$ should not exceed too much the number of sensors. Indeed, when $n$ increases, the level of source interference increases, and hence, the source disjointness assumption is ill satisfied. Moreover, for a large number of sources, the likelihood of having two sources closely spaced, i.e., such that the spatial directions $\mathbf{a}_{i}$ and $\mathbf{a}_{j}$ are "close" to linear dependency, increases. In that case, vector clustering performance degrades significantly. In brief, sparseness and spatial separation are the two limiting factors against increasing the number of sources. 


\subsubsection{Overdetermined case}

The class of UBSS algorithms presented in Section 3.3 can be further simplified in the overdetermined case where $m \geq n$. In that context, the algorithm can be reduced to the mixing matrix estimation step, the noise thresholding then source estimation using the mixing matrix pseudo-inversion.

\subsubsection{Improved BSS using high-resolution MTFDs}

In the case where the received multisensor signal presents high cross-terms, the BSS may become a challenging. Therefore, in order to improve the robustness against cross-terms of separation procedure based on joint diagonalization (JD, JOD and JBD), we can use the hight resolution TFDs introduced in Section 2.3.2 instead of MWVD. Indeed, despite its many desirable properties, the MWVD has some drawbacks that require a precise handling such as cross-terms. Such cross-terms are undesirable in BSS application. Then, cross-terms can be reduced by using high resolution TFDs such as EMBD, CKD or MDD.

To support this claim, let us consider the same three source signals presented in Section 3.1.4 as depicted in the first row of Fig. 24. The generated signals are mixed using an instantaneous noisy uniform linear array model, to be received on $m=6$ sensors with an SNR of $30 \mathrm{~dB}$ (Fig. 25). The received mixtures are whitened, and their MTFD using CKD is computed with parameters $c=1, D=0.1$ and $E=0.1$ for the selection of auto and cross-terms. Finally, the un-mixing matrix is estimated, using a joint diagonalization/joint off-diagonalization algorithm, and estimated sources are classified using their time-frequency correlation with the original signals, as depicted in the second row of Fig. 24. The main objective of this simulation is to illustrate the tradeoff between resolution and cross-terms suppression, when representing different classes of signals such as CKD. Indeed, one can observe from Figs. 12 and 25 that the cross-terms have been significantly reduced with keeping a good timefrequency resolution which improve the robustness of TF BSS algorithms against auto-terms and cross-terms selection. This robustness against auto-terms and cross-terms selection will improve the performance of joint diagonalization al- 

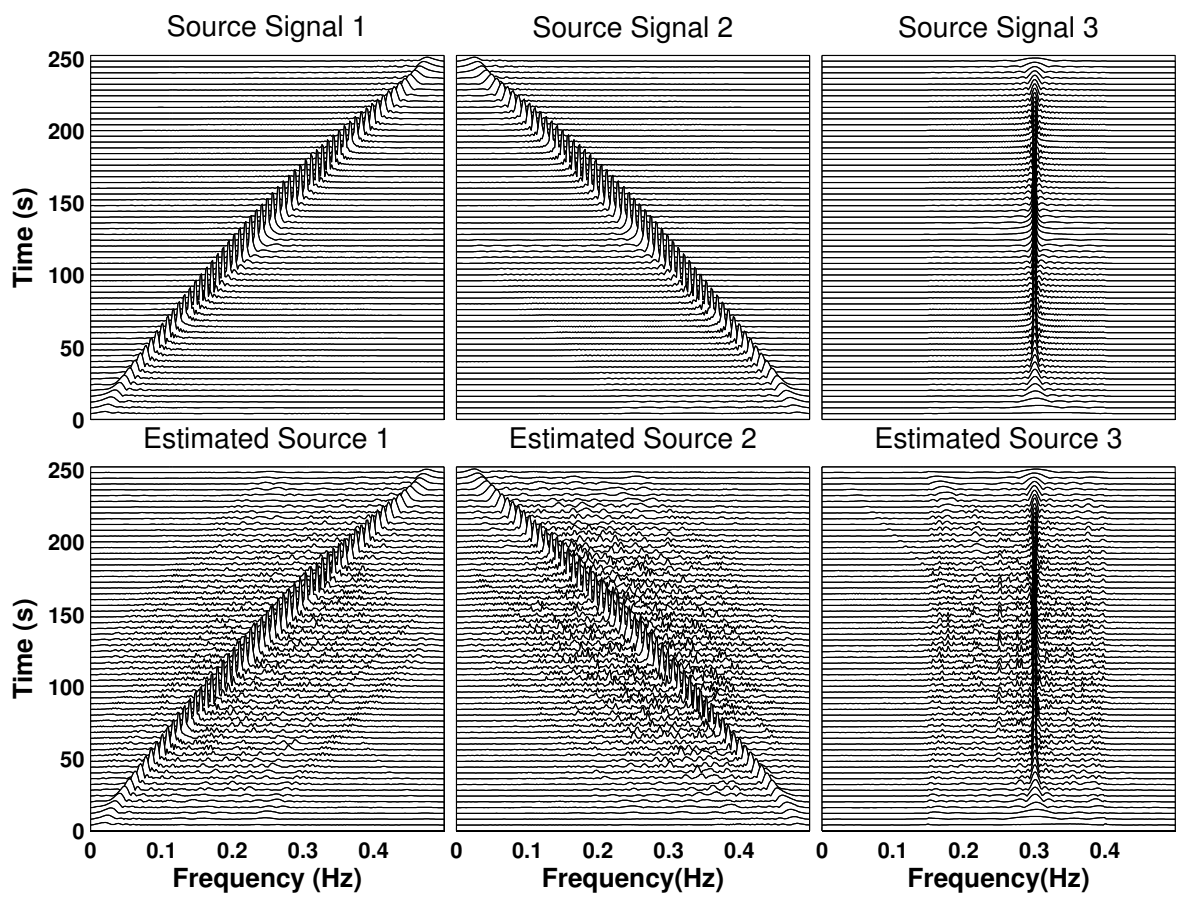

Figure 24: Blind source separation of three source signals $(n=3)$ based on MTFDs: MTFD computed using $C K D$ with parameters $c=1, D=0.1$ and $E=0.1$.

gorithms and then the separation quality (see Fig. 24). The results presented in this section can be reproduced using the codes provided in [5].

\section{Direction of arrival estimation using MTFDs}

\subsection{Background and motivation}

The topic of direction of arrival (DOA) estimation has received considerable attention in past studies over the last three decades (see [54] and [55]). In this section, a brief overview of some of the most important and fundamental approaches to DOA estimation is provided to serve as background for a detailed tutorial presentation of more recent advances.

This section focuses on DOA estimation techniques, starting with the details of traditional time-domain algorithms. These traditional algorithms provide a 


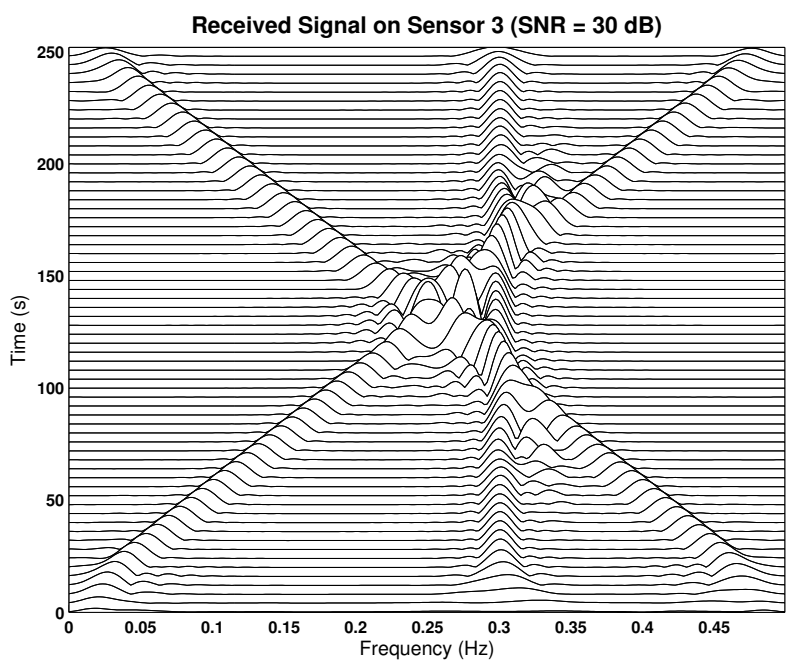

Figure 25: Received mixed signal: Received Signal on Sensor 3 with SNR $=30 d B$ computed using $C K D$ with parameters $c=1, D=0.1$ and $E=0.1$.

basis to the subsequent sections related to more advanced DOA estimation schemes based on multisensor TFDs.

Let us consider a basic scheme of an array as illustrated in Fig. 26. Let $d$ be the distance between any two consecutive elements of the array, $c$ the speed of light, $\Delta t$ the time delay between the elements and $\theta$ the incident angle of the far field signal. The signals arrive at the array elements with a delay $\Delta t$ caused by the path difference i.e.:

$$
\Delta t=\frac{d \cos (\theta)}{c} .
$$

This means that, it takes $\Delta t$ seconds less for one signal to reach an antenna in the two element array relative to the first one. In the frequency domain, such delays appear as a phase shift in the signals received by the elements i.e.:

$$
e^{-j \omega \Delta t}=e^{-j 2 \pi f_{c}\left(\frac{d}{c}\right) \cos (\theta)}=e^{-j 2 \pi f_{c}\left(\frac{d \cos (\theta)}{\lambda f_{c}}\right)}=e^{-j 2 \pi\left(\frac{d}{\lambda}\right) \cos (\theta)},
$$

where $f_{c}$ is the center frequency and $\lambda$ is the wavelength of the signal [55]. With knowledge of the geometry of the array, the delays or phase differences are used to estimate the incident angle. If the time delay of the signal is known, the 


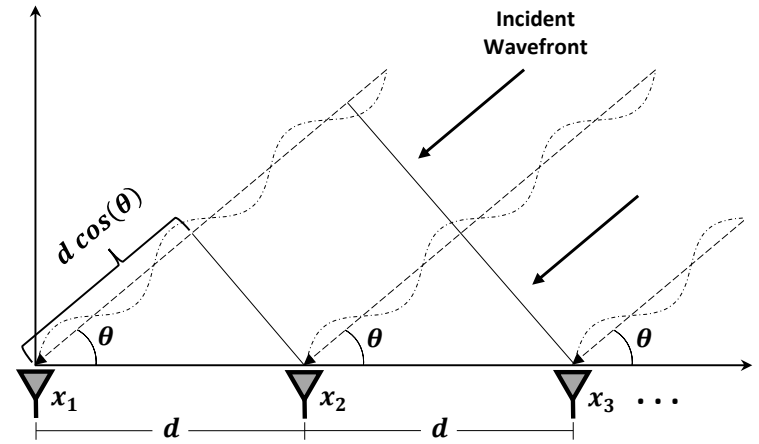

Figure 26: Basic principle of DOA estimation (an array of antenna receiving a signal from a far field source).

direction of the signal is estimated from Eq. (62). This is the basic principle of spatial spectrum estimation techniques.

For an $m$ antennas array, the steering vector given in Eq. (13) can be defined as: $\mathbf{a}\left(\theta_{i}\right)=\left[1, e^{-j 2 \pi\left(\frac{d}{\lambda}\right) \cos \left(\theta_{i}\right)}, e^{-j 2 \pi\left(\frac{2 d}{\lambda}\right) \cos \left(\theta_{i}\right)}, \cdots, e^{-j 2 \pi\left(\frac{(m-1) d}{\lambda}\right) \cos \left(\theta_{i}\right)}\right]$, where $\left\{\theta_{i}\right\}_{i=1}^{n}$ is the angle of arrival of the $i^{\text {th }}$ source signal.

\subsection{Time domain DOAs estimation}

The covariance matrix of the observation vector $\mathbf{z}(t)$ is given by $\mathbf{R}_{z z}=$ $\mathbb{E}\left\{\mathbf{z}(t) \mathbf{z}^{H}(t)\right\}$ such that (see Section 2.3.1):

$$
\mathbf{R}_{z z}=\mathbf{A} \mathbf{R}_{s s} \mathbf{A}^{H}+\sigma_{\eta}^{2} \mathbf{I}_{m} .
$$

Let us consider the case where $\mathbf{R}_{s s}=\mathbb{E}\left\{\mathbf{s}(t) \mathbf{s}^{H}(t)\right\}$ is a full rank matrix. This occurs by assuming (1) non-coherence ${ }^{9}$ of the $n$ incoming signals, and (2) that the set of $n$ vectors in $\mathbf{A}$ are linearly independent. The eigenvalue decomposition of the covariance matrix is then carried out as the DOAs are determined by the eigen-structure of the matrix. Let $\varrho_{1} \geq \varrho_{2} \geq \ldots \geq \varrho_{m}$ denote the eigenvalues of the matrix $\mathbf{R}_{z z}$, and $\lambda_{1} \geq \lambda_{2} \geq \ldots \geq \lambda_{m}$ those of the matrix $\mathbf{A R}_{s s} \mathbf{A}^{H}$, respectively. Eq. (64) yields $\varrho_{i}=\lambda_{i}+\sigma_{\eta}^{2}, \quad i=1,2, \ldots, m$.

\footnotetext{
${ }^{9}$ If $s_{i}(t)$ and $s_{j}(t)$ are non-coherent signals, then $\mathbb{E}\left\{s_{i}(t) s_{j}(t)^{*}\right\}=0$.
} 
Since, the matrix $\mathbf{A}$ is full column rank $n$, the $(m-n)$ smallest eigenvalues of $\mathbf{R}_{z z}$ equal $\sigma_{\eta}^{2}$ :

$$
\varrho_{i}= \begin{cases}\lambda_{i}+\sigma_{\eta}^{2} & i=1,2, \ldots, n, \\ \sigma_{\eta}^{2} & i=n+1, n+2, \ldots, m .\end{cases}
$$

The eigenvalue decomposition of the covariance matrix $\mathbf{R}_{z z}$ then reduces to:

$$
\mathbf{R}_{z z}=\sum_{i=1}^{n}\left(\lambda_{i}+\sigma_{\eta}^{2}\right) \mathbf{v}_{i} \mathbf{v}_{i}^{H}+\sum_{i=n+1}^{m} \sigma_{\eta}^{2} \mathbf{v}_{i} \mathbf{v}_{i}^{H},
$$

where $\mathbf{v}_{i}^{H} \mathbf{v}_{j}=\delta_{i, j}$ are the orthogonal eigenvectors of the matrix $\mathbf{R}_{z z}$ (i.e., $\mathbf{R}_{z z} \mathbf{v}_{i}=\varrho_{i} \mathbf{v}_{i}$ for $\left.i=1,2, \ldots, m\right)$. Eq.(66) will then simplify to $\mathbf{R}_{z z} \mathbf{v}_{i}=$ $\sigma_{\eta}^{2} \mathbf{v}_{i}, \quad i=n+1, n+2, \ldots, m$ or, equivalently,

$$
\left(\mathbf{R}_{z z}-\sigma_{\eta}^{2} \mathbf{I}_{m}\right) \mathbf{v}_{i}=\mathbf{0}, \quad i=n+1, n+2, \ldots, m
$$

Eq.(64) can be rewritten as $\mathbf{R}_{z z}-\sigma_{\eta}^{2} \mathbf{I}_{m}=\mathbf{A} \mathbf{R}_{s s} \mathbf{A}^{H}$; Eq.(67) then becomes: $\mathbf{A R}_{s s} \mathbf{A}^{H} \mathbf{v}_{i}=\mathbf{0}, \quad i=n+1, n+2, \ldots, m$, which yields:

$$
\mathbf{A}^{H} \mathbf{v}_{i}=\mathbf{0}, \quad i=n+1, n+2, \ldots, m .
$$

Eq.(68) shows that the subspace spanned by the eigenvectors $\left\{\mathbf{v}_{n+1}, \mathbf{v}_{n+2}, \ldots, \mathbf{v}_{m}\right\}$ is orthogonal to the complement subspace spanned by the steering vectors in matrix $\mathbf{A}$. Therefore, from the eigenvectors of $\mathbf{R}_{z z}$, one can obtain the signal DOAs by finding those steering vectors that are orthogonal to the noise subspace.

\subsubsection{Time Domain MUSIC Algorithm}

Spectral-based DOA estimation methods are based on maximizing the power of signal projected on the signal subspace. One of the standard DOA techniques, MUSIC (Multiple SIgnal Classification) [56], performs an eigenvalue decomposition of the unknown covariance matrix $\mathbf{R}_{z z}$ estimated as:

$$
\widehat{\mathbf{R}}_{z z}=\frac{1}{T} \int_{0}^{T} \mathbf{z}(\tau) \mathbf{z}^{H}(\tau) d \tau,
$$


where $T$ is the signal duration. Let use express the eigenvector estimates as $\left\{\widehat{\mathbf{v}}_{1}, \widehat{\mathbf{v}}_{2}, \ldots, \widehat{\mathbf{v}}_{m}\right\}$ such that the singular value decomposition of $\widehat{\mathbf{R}}_{z z}$ is given by:

$$
\widehat{\mathbf{R}}_{z z}=\widehat{\mathbf{V}} \boldsymbol{\Lambda} \widehat{\mathbf{V}}^{H}
$$

with $\widehat{\mathbf{V}}=\left[\widehat{\mathbf{v}}_{1}, \widehat{\mathbf{v}}_{2}, \ldots, \widehat{\mathbf{v}}_{m}\right]$. Using Eq.(68), MUSIC then estimates the signal directions as the peaks of the spatial spectrum estimate expressed by [55]:

$$
P_{\text {MUSIC }}(\theta)=\frac{1}{\sum_{i=n+1}^{m}\left|\mathbf{a}(\theta) \widehat{\mathbf{v}}_{i}\right|^{2}}
$$

where $\mathbf{a}(\theta)$ is a column vector of the steering matrix defined by Eq.(13). The MUSIC spectrum is estimated from a single realization of the random process represented by the observations $\mathbf{z}(t)$ for $t=1,2, \ldots, T$. MUSIC estimates were shown to be consistent and they converge to the true source bearings as the number of observations increases to infinity [57].

\subsubsection{Time Domain ESPRIT Algorithm}

ESPRIT stands for Estimation of Signal Parameters via Rotational Invariance Techniques $[58,54]$. The aim of the ESPRIT algorithm is to exploit the rotational invariance in the signal subspace which is created by two arrays with a translational invariance structure. ESPRIT inherently assumes narrowband signals so as to know the translational phase relationship between the multiple arrays to be used. The ESPRIT algorithm is more robust with respect to array imperfections than MUSIC [59,60]. Computation complexity and storage requirements are lower than MUSIC as it does not perform an extensive search throughout all possible steering vectors [54]. The ESPRIT algorithm is summarized as follows:

- Estimate the correlation matrix $\widehat{\mathbf{R}}_{z z}$ by using Eq. (69) and compute its eigendecomposition in order to get the eigenvector $\left\{\widehat{\mathbf{v}}_{1}, \widehat{\mathbf{v}}_{2}, \ldots, \widehat{\mathbf{v}}_{m}\right\}$.

- From the $n$ principal eigenvectors $\left\{\widehat{\mathbf{v}}_{1}, \widehat{\mathbf{v}}_{2}, \ldots, \widehat{\mathbf{v}}_{n}\right\}$, representing the signal subspace, form the matrix $\mathbf{V}_{s}=\left[\widehat{\mathbf{v}}_{1}, \widehat{\mathbf{v}}_{2}, \ldots, \widehat{\mathbf{v}}_{n}\right]$. Then, from the matrix 
$\mathbf{V}_{s}$ form the matrices $\mathbf{V}_{1}$ and $\mathbf{V}_{2}$ such that:

$$
\mathbf{V}_{s}=\left[\begin{array}{c}
\mathbf{V}_{1} \\
\text { last row }
\end{array}\right]=\left[\begin{array}{c}
\text { first row } \\
\mathbf{V}_{2}
\end{array}\right] \text {. }
$$

- Solve in a least square sense the relation $\mathbf{V}_{2}=\mathbf{V}_{1} \boldsymbol{\Psi}$ in order to estimate the matrix $\boldsymbol{\Psi}$.

- Find the eigenvalues $\left\{\psi_{1}, \psi_{2}, \ldots, \psi_{n}\right\}$ of the matrix $\boldsymbol{\Psi}$. Then, DOA estimates are obtained by [55]:

$$
\theta_{i}=\arccos \left(\frac{j \lambda \log \psi_{i}}{2 \pi d}\right)
$$

\subsection{Time-frequency DOAs estimation}

\subsubsection{Time-Frequency MUSIC Algorithm}

As noted in Section 2.3.1, the linear model given in Eq. (21) has the same structure as the covariance matrix based on the linear model given in Eq. (64). This similarity suggests that the MUSIC algorithm can be simply extended for direction finding using the subspace decomposition of an averaged MTFD matrix. The steps needed to find the averaged MTFD matrix $\boldsymbol{\rho}_{z z}$ are given in Table 1. After estimating the averaged MTFD matrix $\boldsymbol{\rho}_{z z}$, TF-MUSIC can therefore simply extend Eq.(71) to get:

$$
P_{\mathrm{TF}-\mathrm{Music}}(\theta)=\frac{1}{\sum_{i=n+1}^{M}\left|\mathbf{a}(\theta) \widehat{\mathbf{v}}_{\mathrm{TF}_{i}}\right|^{2}},
$$

where $\widehat{\mathbf{v}}_{\mathrm{TF}_{i}}$ is the $i^{\text {th }}$ eigenvector of the averaged MTFD matrix $\boldsymbol{\rho}_{z z}$. A difference between the averaged MTFD matrix $\boldsymbol{\rho}_{z z}$ and the sample covariance $\widehat{\mathbf{R}}_{z z}$ is that the former is obtained by averaging selected high signal energy points by rejecting noise contributions while the later is obtained by averaging all available points including noise contributions. Hence, the averaged MTFD matrix $\boldsymbol{\rho}_{z z}$ based directional estimation technique $P_{\mathrm{TF}-\mathrm{MUSIC}}(\theta)$ is expected to improve performance. In addition, the TF approach allows the estimation of the covariance matrix of each source separately (e.g. by using a multi-component IF 
Table 1: Algorithm to estimate the averaged MTFD matrix

1. Compute the MTFD matrix $\boldsymbol{\rho}_{z z}(t, f)$ as given in Eq.(21).

2. Select auto-TFDs $\left\{\rho_{z_{i, i}}(t, f)\right\}_{i=1}^{m}$ and compute the spatial averaged TFD as:

$$
\rho_{\text {avg }}(t, f)=\frac{1}{m} \sum_{i=1}^{m} \rho_{z_{i, i}}(t, f) .
$$

3. Select high energy $(t, f)$ points as Eq.(52):

$$
\text { Select the point }\left(t_{i}, f_{i}\right) \text { if }\left\|\rho_{a v g}\left(t_{i}, f_{i}\right)\right\|>\epsilon_{t}
$$

and reject the $(t, f)$ points with negligible energy (e.g. noise) to improve the SNR. The threshold $\epsilon_{t}$ is a user defined parameter; in this study the value for $\epsilon_{t}$ is selected such that: $\epsilon_{t} \geq 0.05 \times \max \left(\rho_{\text {avg }}\right)$.

4. Compute the averaged MTFD matrix $\boldsymbol{\rho}_{z z}$ using averages of the selected $(t, f)$ points in the previous step as:

$$
\boldsymbol{\rho}_{z z}=\frac{1}{n_{\text {points }}} \sum_{i=1}^{n_{\text {points }}} \boldsymbol{\rho}_{z z}\left(t_{i}, f_{i}\right) .
$$

where $n_{\text {points }}$ represents the total number of selected $(t, f)$ points.

approach). This is significant as it then allows the formulation of estimates of DOAs even in the case where there are more sources than sensors (case discussed later in Section 4.4).

\subsubsection{Time-Frequency ESPRIT Algorithm}

As for TF-MUSIC, one can extend the ESPRIT algorithm to TF-ESPRIT for direction finding based on MTFD matrices by considering the subspace decomposition of an averaged MTFD matrix (see Table 1). After estimating the averaged MTFD matrix $\boldsymbol{\rho}_{z z}$, TF-ESPRIT uses $\boldsymbol{\rho}_{z z}$ instead of $\hat{\mathbf{R}}_{z z}$ in the subspace technique given in Section 4.1.2 to get the DOA estimates. The advantage of this approach, like TF-MUSIC, is to exploit the accuracy and robustness of the averaged MTFD matrix $\boldsymbol{\rho}_{z z}$ over the basic sample covariance $\hat{\mathbf{R}}_{z z}$ approach. 


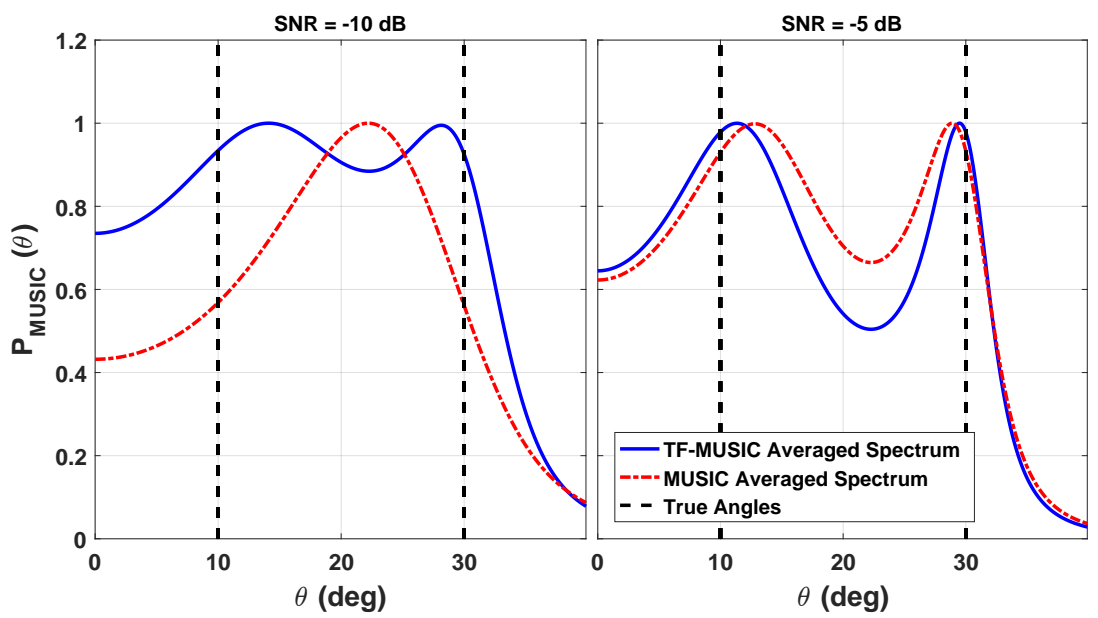

Figure 27: DOA estimation of two sources using MUSIC and TF-MUSIC (average spatial spectrum).

\subsection{Examples}

Let us compare the performance of time-frequency DOA estimation methods and the conventional DOA estimation methods, for the case of a uniform linear array having eight sensors $(m=8)$. Two source signals arrive on this array from directions $\theta_{1}=10^{\circ}$ and $\theta_{2}=30^{\circ}$, respectively. For the time-frequency DOA estimation methods, we have used the MTFD based on WVD. In Fig. 27 the estimated spatial spectra of the TF-MUSIC and conventional time-domain MUSIC are shown for SNRs $-5 \mathrm{~dB}$ and $-10 \mathrm{~dB}$. The results indicate that TFMUSIC outperforms its time-domain counterpart in resolving two closely spaced sources.

Figs. 28 and 29 represent the histograms of estimated angles by using MUSIC, TF-MUSIC, ESPRIT and TF-ESPRIT for SNRs $-10 \mathrm{~dB}$ and $-5 \mathrm{~dB}$ respectively. The results indicate that TF methods outperforms their time-domain counterparts in terms of error estimation. This conclusion, is confirmed by Fig. 30 which represents the normalized mean square error of angle estimation with respect to SNR for MUSIC, TF-MUSIC, ESPRIT and TF-ESPRIT algorithms. 


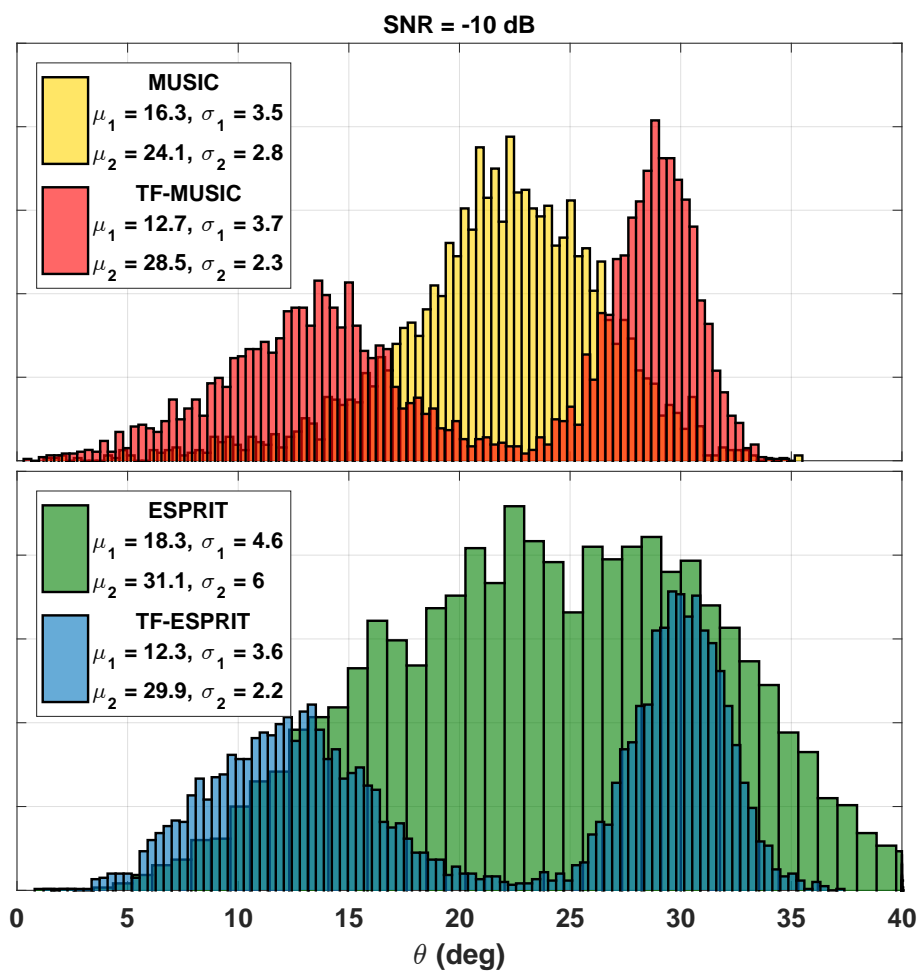

Figure 28: DOA estimation of two sources using MUSIC, TF-MUSIC, ESPRIT and TFESPRIT for SNR=-10dB (histogram of estimated angles): TF-MUSIC and TF-ESPRIT provide a more accurate DOA estimate than MUSIC and ESPRIT, respectively.

The above results can be reproduced using the codes provided in [5].

\subsection{Underdetermined DOAs estimation using MTFDs}

Another advantage of TF source localization is its ability to address the problem of sources localization in the underdetermined case [61]. The TF approach allows the estimation of the covariance matrix of each source separately, and therefore yields estimates of DOAs, even in the case of more sources than sensors. Indeed, as presented in Section 3.3.2 and based on clustering information we can define a time-frequency binary mask to separate the TF region where each source is present alone. Based on the clustering information and 


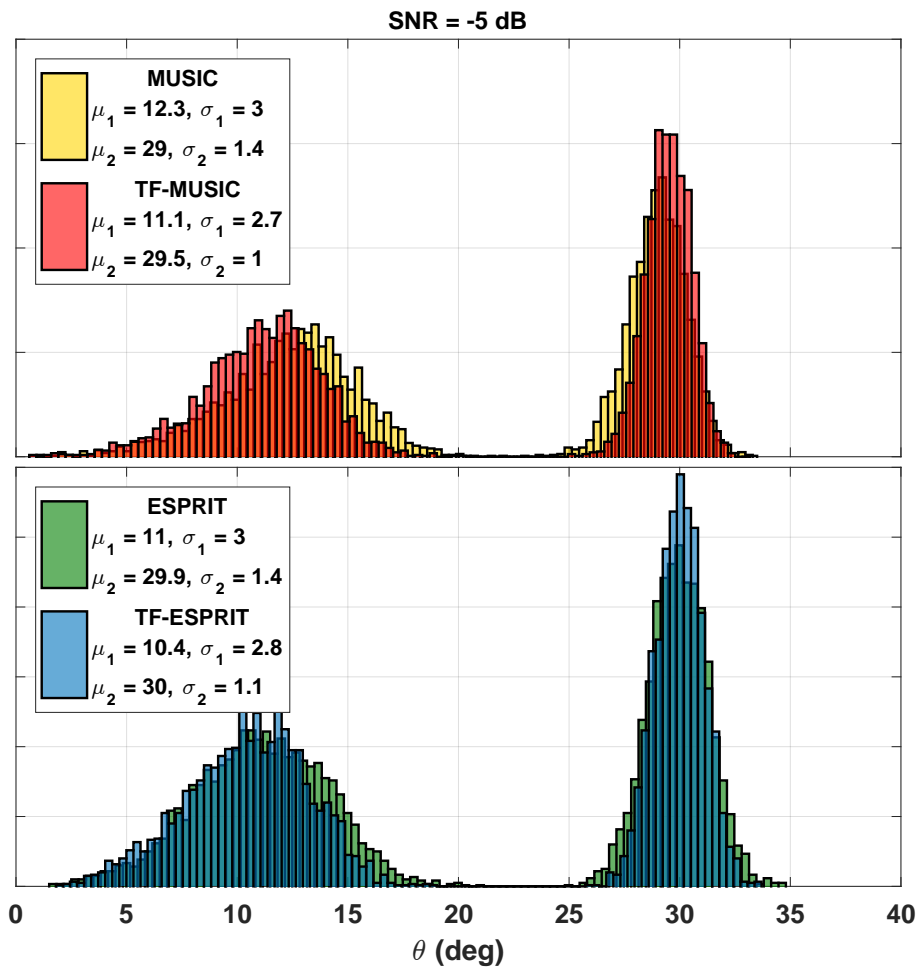

Figure 29: DOA estimation of two sources using MUSIC, TF-MUSIC, ESPRIT and TFESPRIT for $\mathrm{SNR}=-5 \mathrm{~dB}$ (histogram of estimated angles): TF-MUSIC and TF-ESPRIT provide a more accurate DOA estimate than MUSIC and ESPRIT, respectively.

the binary masking, one can use the TF-DOA estimation algorithms presented in Section 4.2 to estimate the DOA of each source. After a clustering step, one can apply the TF binary masking operation defined as:

$$
\widehat{\boldsymbol{\rho}}_{s_{i}, s_{i}}(t, f)=\boldsymbol{\rho}_{z z}(t, f) \Omega_{i}(t, f),
$$

such that:

$$
\Omega_{i}(t, f)= \begin{cases}1, & \text { if }(t, f) \in \mathcal{C}_{i} \\ 0, & \text { otherwise. }\end{cases}
$$

where $\widehat{\boldsymbol{\rho}}_{s_{i}, s_{i}}$ is the estimated TFD of the $i^{t h}$ source, $\boldsymbol{\rho}_{z z}$ represents the averaged MTFD introduced in Section 4.2.1. 


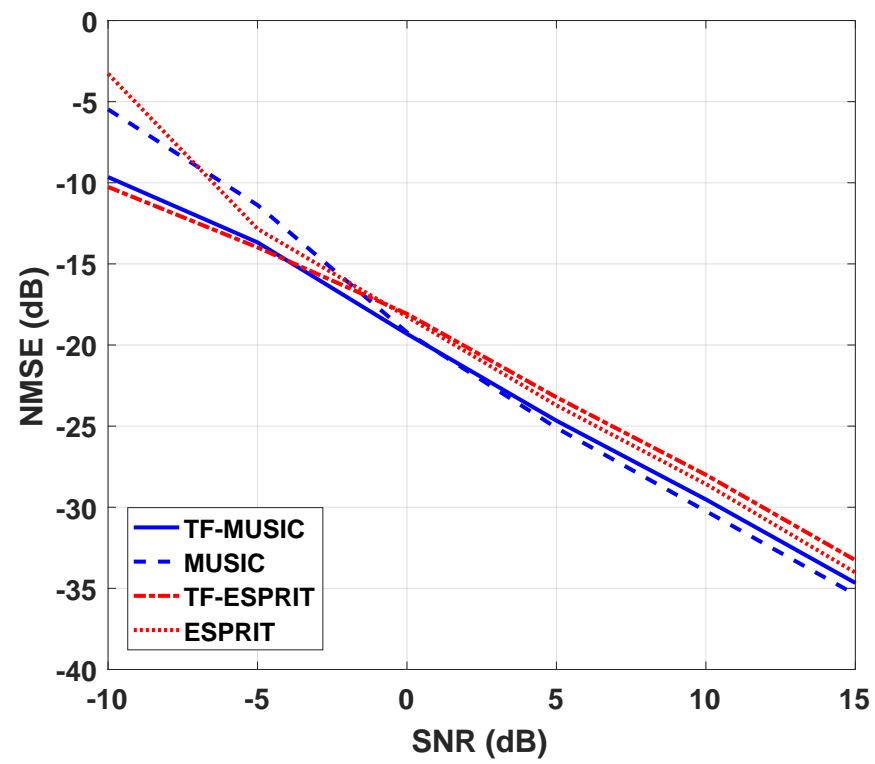

Figure 30: Normalized mean square error of DOA estimation with respect to SNR.

\subsection{Discussion}

The performance and limitations of the above DOA estimation algorithms and their applications are further discussed below.

\subsubsection{Signal subspace dimension}

In the presented DOA estimation methods the number of sources $n$ is assumed known. Then, an important problem is the determination of $n$, the number of source signals. Based on the fact that the number of source signals is equal to the number of large eigenvalues of the covariance matrix, one can obtain relatively simple non-parametric algorithms for estimating $n$. The idea is to determine the multiplicity of the smallest eigenvalue, which theoretically equals $m-n$. A statistical hypothesis test can be used based on information theoretic criteria, such AIC and MDL [47]. The estimation of the number of 
sources can be done by using Akaike's criterion according to:

$$
\widehat{n}_{\mathrm{AIC}}=\underset{k}{\arg \min }\left\{T(m-k) \log \left(\frac{\sum_{i=k+1}^{m} \varrho_{i}}{(m-k) \prod_{i=k+1}^{m} \varrho_{i}^{\frac{1}{m-k}}}\right)+k(2 m-k)\right\} .
$$

or using MDL criterion according to:

$$
\widehat{n}_{\mathrm{MDL}}=\underset{k}{\arg \min }\left\{T(m-k) \log \left(\frac{\sum_{i=k+1}^{m} \varrho_{i}}{(m-k) \prod_{i=k+1}^{m} \varrho_{i}^{\frac{1}{m-k}}}\right)+(k(2 m-k)+1) \log (\sqrt{T})\right\} .
$$

where value $\varrho_{i}, i=1, \ldots, m$ represent the eigenvalues of the covariance ma-

trix $\widehat{\mathbf{R}}_{z z}$. Unfortunately, the aforementioned approach is very sensitive to the assumption of a spatially white noise field [62].

Let us consider the case of a uniform linear array having six sensors $(m=6)$. Two source signals $(n=2)$ arrive on this array from directions $\theta_{1}=10^{\circ}$ and $\theta_{2}=30^{\circ}$, respectively. Fig. 31 represents the estimated number of sources with respect to the SNR for AIC and MDL criteria. We can observe that both methods well estimated the number of sources from SNR grater thant $-4 \mathrm{~dB}$ with a bias for AIC method. The same observation can be made for Fig. 32, where we consider the case of a uniform linear array having six sensors $(m=6)$ and three source signals $(n=3)$ arrive on this array from directions $\theta_{1}=10^{\circ}$, $\theta_{2}=30^{\circ}$ and $\theta_{3}=50^{\circ}$, respectively.

\subsubsection{Spatial resolution}

The spatial resolution is defined as the ability to distinguish two or more sources with very close incident angle. As for MUSIC and TF-MUSIC algorithms, the resolution ability is one of their weakness. It is not hard to understand that we cannot decide the exact number of signal from one peak in the graph of MUSIC and TF-MUSIC algorithms. A small step of scanning can improve the resolution ability but cannot solve this problem totally. However, 


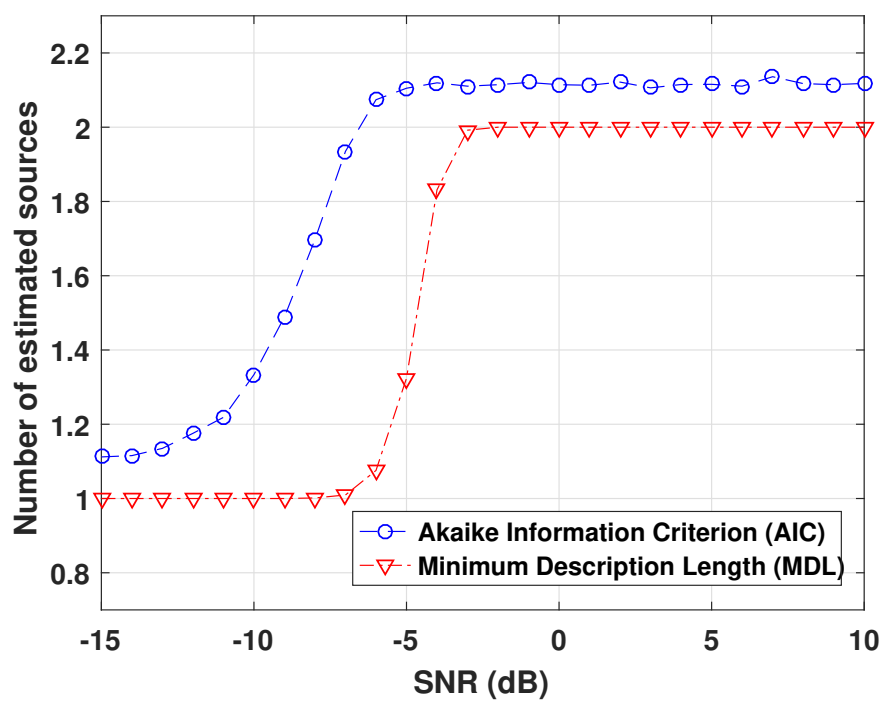

Figure 31: Estimated number of sources with respect to SNR for $n=2$ sources and $m=6$ sensors using AIC and MDL criteria.

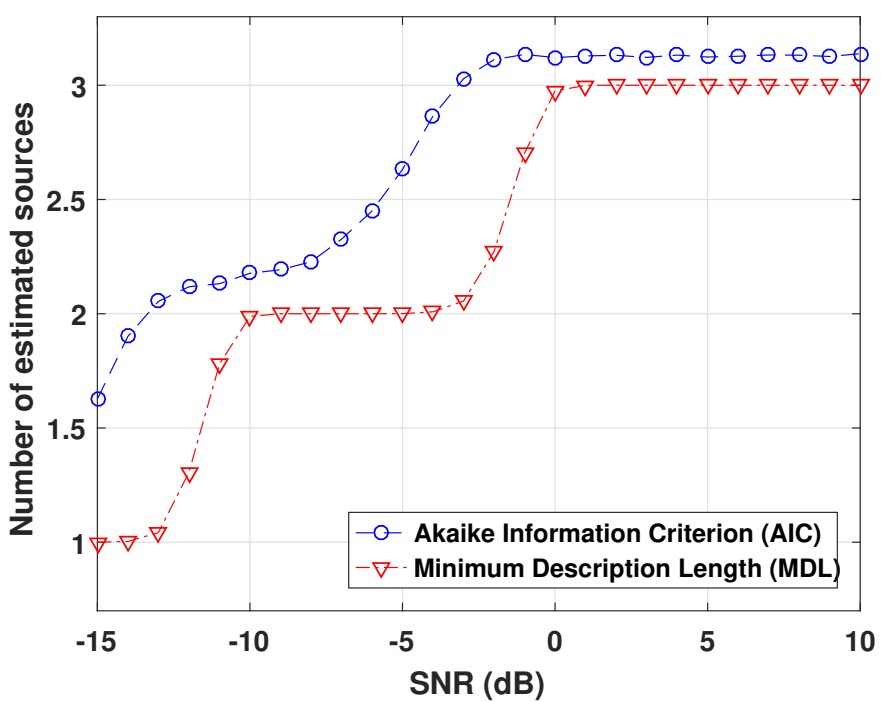

Figure 32: Estimated number of sources with respect to SNR for $n=3$ sources and $m=6$ sensors using AIC and MDL criteria. 


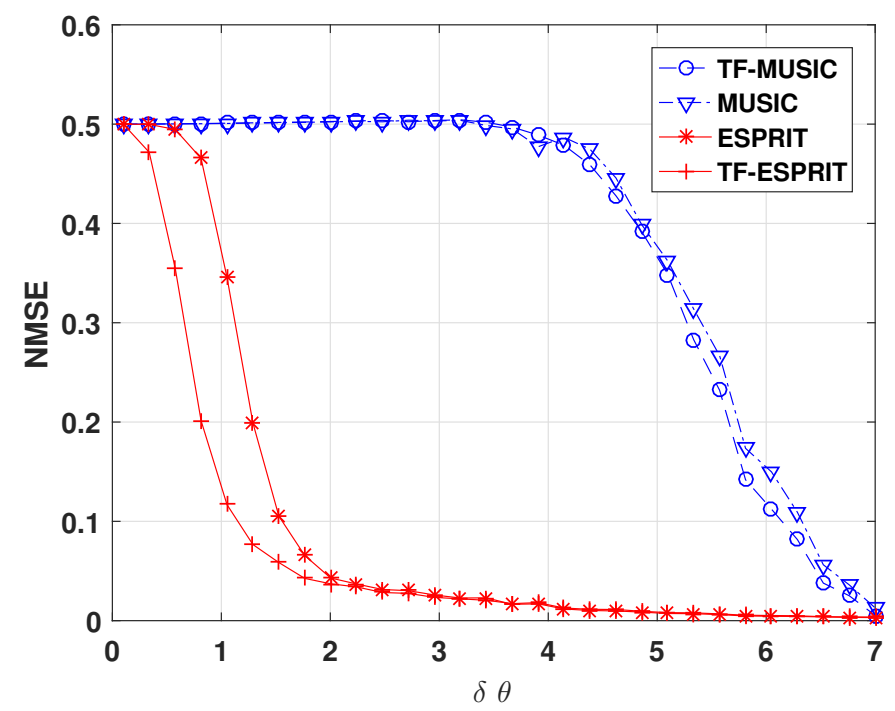

Figure 33: Normalized mean square error of DOA estimation with respect to spatial resolution between the sources $\delta \theta$ : ESPRIT and TF-ESPRIT provide a more accurate DOA estimate than MUSIC and TF-MUSIC, respectively.

ESPRIT and TF-ESPRIT have excellent spatial resolution due to the fact that they estimate the DOA from an analytic expression (see Section 4.1.2).

To support this claim, let us compare the performance of MUSIC, TFMUSIC, ESPRIT and TF-ESPRIT, for the case of a uniform linear array having six sensors $(m=6)$. Two source signals arrive on this array from directions $\theta_{1}=10^{\circ}$ and $\theta_{2}=10^{\circ}+\delta \theta$, respectively. Fig. 33 represents the normalized mean square error of angle estimation with respect to the spatial resolution $\delta \theta$ for MUSIC, TF-MUSIC, ESPRIT and TF-ESPRIT algorithms and for SNR 20dB. We can observe that ESPRIT and TF-ESPRIT algorithms outperform MUSIC and TF-MUSIC in resolving two closely spaced sources.

\subsubsection{Improved DOA estimation using high-resolution MTFDs}

As presented in Section 4.2, the TF DOA algorithms are based on the average MTFD matrix instead of the covariance matrix. The averaged MTFD matrix $\boldsymbol{\rho}_{z z}$ is obtained by averaging selected high signal energy points by rejecting 
noise contributions. However, in the case where the received signal presents high cross-terms, the averaged MTFD matrix $\boldsymbol{\rho}_{z z}$ can be disturbed by these cross-terms and then degrade the performance of DOA estimations. Therefore, in order to improve the robustness against cross-terms of TF DOA algorithms, we can use the hight resolution TFDs introduced in Section 2.3.2 instead of MWVD. Indeed, the high resolution TFDs provide a good compromise between resolution and cross-terms reduction which improve the robustness of TF DOA algorithms against cross-terms.

To support this claim, let us compare the performance of the conventional MUSIC algorithm, TF-MUSIC algorithm using different TFDs, for the case of a uniform linear array having eight sensors $(m=4)$. Two source signals arrive on this array from directions $\theta_{1}=-5^{\circ}$ and $\theta_{2}=5^{\circ}$, respectively. For the TF-MUSIC algorithm, we have used the MTFD based on WVD and MDD respectively. In Fig. ??a the estimated spatial spectra of the TF-MUSIC based on MDD is shown, while Fig. ??b shows the estimated spatial spectra of the for conventional MUSIC (red) and TF-MUSIC based on the MWVD (black). The results indicate that, when the SNR is set to $-10 \mathrm{~dB}$ both standard TF-MUSIC based on the MWVD and conventional MUSIC fail to estimate the 2 angles accurately (see Fig. ??b), while the TF-MUSIC based on MDD gives sharp peaks at the incident angles as shown in Fig. ??a. In the second experiment, keeping the same setup with an SNR of $10 \mathrm{~dB}$, all the algorithms appear to give sharp peaks at estimated incident angles as illustrated in Fig. ??c for the TFMUSIC based on MDD and Fig. ??d for the TF-MUSIC based on the MWVD and conventional MUSIC algorithms.

\section{Cross Channel Causality Analysis}

\subsection{Background and motivation}

Previous sections have shown that by combining array signal processing for non-stationary signals and multichannel high resolution time-frequency signal processing methods, one can enhance the performance of several standard meth- 
ods and techniques (such as BSS and DOA estimation) for a wide field of applications by simultaneously taking into account (1) the non-stationary characteristics of measured signals using TFDs and (2) the spatial information provided by an array of measuring sensors.

In addition to such improvements, another key capability of the MTFSP approach is to allow a signal causality analysis across sensors/channels and/or signals; new information is then gained e.g. by tracking the time varying location of moving sources propagating through the system. Combining spatial and timefrequency information obtained by a multisensor array results then in improved precision. Examples are:

(1) in brain studies, the concept of cross channel causality can be used to characterize the propagation of a seizure location across EEG channels, therefore providing a key information about the time-varying information flow scalp [26];

(2) In wireless communication, it characterizes the varying spatial location of a moving user (mobile) in cell by exploiting the multi-antenna array of the base station;

(3) In aeronautics, a primary design goal for helicopter engineers is to utilize vibrations measured from a multichannel system to identify the excitation sources or faults of a rotating system [63].

\subsection{Cross-channel causality and phase synchrony}

The cross channel causality describes the dependence relationship between multichannel signals. This dependence can be quantified using various approaches, from simple linear correlation to more advanced TF based approaches. Cross-correlation between two zero mean signals $x_{i}(t)$ and $x_{j}(t)$ under the weaksense stationarity assumption is defined as:

$$
r_{i j}(\tau)=\frac{\mathbb{E}\left\{x_{i}(t) x_{j}(t+\tau)^{*}\right\}}{\sqrt{\mathbb{E}\left\{x_{i}(t)^{2}\right\} \mathbb{E}\left\{x_{j}(t+\tau)^{2}\right\}}} \text { such that } \quad-1 \leq r_{i j}(\tau) \leq 1 .
$$

The cross-correlation is typically utilized to assess the linear relationship between different signals; however, a simple correlation coefficient at the zeroth 
lag is limited in its ability to assess the interactions of these signals, as it can only quantify the linear relationships and capture mostly the amplitude-based relationships.

Another useful measure between multichannel signals is the coherence function defined as:

$$
C_{i j}(f)=\frac{\mathcal{S}_{i j}(f)}{\sqrt{\mathcal{S}_{i i}(f) \mathcal{S}_{j j}(f)}} \quad \text { such that } \quad 0 \leq\left|C_{i j}(f)\right| \leq 1 .
$$

where $\mathcal{S}_{i j}(f)$ is the cross-spectral density between $x_{i}(t)$ and $x_{j}(t)$ given by:

$$
\mathcal{S}_{i j}(f)=\underset{\tau \rightarrow f}{\mathcal{F}}\left\{\mathbb{E}\left\{x_{i}(t) x_{j}(t+\tau)^{*}\right\}\right\}
$$

and $\mathcal{S}_{i i}(f)$ and $\mathcal{S}_{j j}(f)$ the auto-spectral density of $x_{i}(t)$ and $x_{j}(t)$ respectively. The coherence function provides a measure that quantifies linear relationships in the frequency domain between two wide sense stationary signals $[64,65]$. The magnitude squared coherence function measures the degree to which one signal can be represented as the output of a linear filter operating on the other signal and varies from 0 for two statistically independent signals to 1 , when one signal is the result of linear filtering performed on the other. The coherence function finds many useful applications but the results based on coherence depend on several factors like stationarity of the signal, segment length, number of segments, etc. [66]. A short but broad review that includes linear as well as non-linear measures is given in $[67,68]$.

In order to mitigate these limitations and address the analysis of nonstationary signal, phase synchrony analysis can be used [69]. Phase synchrony analysis is a useful measure of linear dependence between multichannel signals. This approach is based on the concept of phase synchronization of chaotic oscillators $[70,71]$. The phase synchrony (coefficient) takes on values between 0 , for two signals at different frequencies, and 1, for signals that exhibit a constant difference in instantaneous phase (representing the situation where a signal and its time-shifted version are observed). So, phase synchrony refers to the interdependence between the instantaneous phases of two signals; the instantaneous 
phases may be strongly synchronized even when the amplitudes of the two signals are statistically independent [70, 71].

The degree of synchrony between two signals is usually assessed via estimation of instantaneous phase around a particular frequency, which is usually accomplished via the Hilbert transform such that [72]:

$$
\varphi(t)=\arg [z(t)]=\arg [x(t)+j \mathcal{H}\{x(t)\}]
$$

Then, in the case of non-stationary signals, TF methods become naturally more suitable for the calculation of synchrony between two signals [73, 74]. Several approaches exist. A Morlet wavelet-based method was used in [75], but here for continuity with above sections, we present a recently proposed method based on quadratic TFDs [73] [1, Section 16.4].

\subsubsection{Phase synchrony estimation using a complex TFD}

Let us consider the QTFD of a signal based on the reduced-interference Rihaczek TFD, defined for a monosensor signal $z(t)$ as:

$$
\rho_{z}(t, f)=\iint_{\mathbb{R}^{2}} e^{-\frac{\nu^{2} \tau^{2}}{\sigma}} e^{-j \pi \nu \tau} \mathcal{A}_{z}(\nu, \tau) e^{-j 2 \pi(f \tau-\nu t)} d \nu d \tau
$$

where $\mathcal{A}_{z}(\nu, \tau)$ is the ambiguity function defined in Section 2.3.2 and given by:

$$
\mathcal{A}_{z}(\nu, \tau)=\int_{\mathbb{R}} z\left(t+\frac{\tau}{2}\right) z^{*}\left(t-\frac{\tau}{2}\right) e^{-j 2 \pi \nu t} d t
$$

The factor $e^{-j \pi \nu \tau}$ is the kernel for the Rihaczek distribution, and $e^{-\frac{\nu^{2} \tau^{2}}{\sigma}}$ is an exponential kernel used to reduce the effect of the cross-terms. Other kernels can be used as long as they remove the cross-terms in the Rihaczek amplitude spectrum.

Therefore, by extending the previous formulation of Rihaczek distributions, the Rihaczek MTFD for the signal vector $\mathbf{z}(t)$ can be defined as:

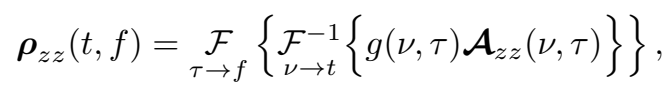

where $\mathcal{A}_{z z}(\nu, \tau)$ is the spatial ambiguity function defined by Eq.(26) and $g(\nu, \tau)$ is the Doppler-lag kernel defined by:

$$
g(\nu, \tau)=e^{-\frac{\nu^{2} \tau^{2}}{\sigma}} e^{-j \pi \nu \tau}
$$


Since the TFD localizes spectral components in the $(t, f)$ domain, the cross correlation of specific events on two spatially separated TFDs gives the phase difference and hence the phase synchrony can be estimated. The amplitude of the cross TFD indicates coincident or partly overlapped signals. The phase of the cross TFD at local maxima indicates the phase difference between them. The phase of the TFD product of two signals $z_{i}(t)$ and $z_{j}(t)$ is then defined as follows [73] [1, Section 16.4]:

$$
\varphi_{i j}(t, f)=\arg \left[\rho_{z_{i}, z_{i}}(t, f) \rho_{z_{j}, z_{j}}^{*}(t, f)\right]
$$

Based on the estimate of a time-varying phase spectrum as shown above, a synchrony measure still needs to be defined. For this, the phase-locking value (PLV) is considered. The PLV between two signals, averaged across realizations/trials, can be defined as [73]:

$$
\mathcal{P}_{i j}(t, f)=\frac{1}{\operatorname{Tr}}\left|\sum_{k=1}^{T r} e^{j \varphi_{i j}^{(k)}(t, f)}\right|
$$

where $\mathcal{P}_{i j}(t, f)$ is the $i j^{t h}$ element of the matrix $\mathcal{P}(t, f), \operatorname{Tr}$ is the number of trials/realizations, and $\varphi_{i j}^{(k)}(t, f)$ is the time-varying phase estimate between $z_{i}(t)$ and $z_{j}(t)$ for the $k^{\text {th }}$ trial. The PLV measures the intertrial/interrealization variations of phase differences at time $t$ and frequency $f$. A PLV close to 1 indicates a small phase difference across trials/realizations. For a single trial, a so-called single-trial PLV is calculated, denoting the consistency of the phase across time. Lastly, the described phase synchrony measure assesses the instantaneous phase differences between signals in the $(t, f)$ domain using the complex Rihaczek distribution [76]. In some applications, such as biomedical signal processing, one would expect to have a "number" which shows whether the channels are synchronized. Then, based on the PLV definition, the channel synchronization can be quantified by using the mean value over time and frequency, such that:

$$
\overline{\mathcal{P}}_{i j}=\frac{1}{\operatorname{Card}\{\Omega\}}\left|\sum_{(t, f) \in \Omega} e^{j \varphi_{i j}(t, f)}\right|,
$$

where $\overline{\mathcal{P}}_{i j}$ is the $i j^{\text {th }}$ element of the mean PLV matrix $\overline{\mathcal{P}}$ which quantifies the synchronization between the $i^{\text {th }}$ and the $j^{\text {th }}$ sensors. 


\subsubsection{Phase synchrony estimation using MTFDs}

The above derivation shows that we can use the cross spectral property to estimate the PLV between two signals as a function of $t$ and $f$. Therefore, a similar attempt can be made by taking advantage of MTFDs definition and using the cross TFD terms to define the phase of the cross TFD as:

$$
\bar{\varphi}_{i j}(t, f)=\arg \left[\rho_{z_{i,}, z_{j}}(t, f)^{2}\right]
$$

This definition, calculates the phase of the square of cross TFD in order to remain homogeneous with respect to the phase definition given by Eq. (90) and to avoid the sign indeterminacy in the real case. Then, the PLV can be estimated from this new definition of phase of cross TFD and following the definition given in Eq. (91).

The method of a time-varying phase estimation presented in Eq. (90) requires the use of the Rihaczek distribution, due to the fact that the Rihaczek distribution is a complex distribution. However, one of the disadvantages of the Rihaczek distribution is the existence of cross-terms for multicomponent signals. These cross-terms are located at the same time and frequency locations as the original signals and will lead to biased energy and phase estimates. To get rid of these cross-terms a reduced interference version of the Rihaczek distribution is used in Eq. (86) by applying a kernel function to filter the cross-terms in the ambiguity domain. On the other hand, the method of a time-varying phase estimation presented in Eq. (93) allows us to exploit the whole class of TFDs which is the fact that the cross TFDs are intrinsically complex and convey a phase information. Therefore, this time-varying phase estimation method enables to choose the best TFD according to the used signals in order to optimize the trade-off between cross-terms reduction and resolution.

In order to assess the behaviour of phase synchrony and PLV, let consider a simple case of two phase locked signals $z_{i}(t)$ and $z_{j}(t)$ such that $z_{j}(t)=z_{i}(t) e^{j \phi}$. In this case, one can observe that $\rho_{z_{i}, z_{i}}(t, f)=\rho_{z_{j}, z_{j}}(t, f)$. Then, the phase of the TFD product of the two signals $z_{i}(t)$ and $z_{j}(t)$ define in Eq. (90) will be:

$$
\varphi_{i j}(t, f)=\arg \left[\rho_{z_{i}, z_{i}}(t, f) \rho_{z_{j}, z_{j}}^{*}(t, f)\right]=0
$$


Therefore, the PLV defined by Eq. (92) tends to 1 which is consistent under phase locked signal assumption. In other hand, the cross TFD of $z_{i}(t)$ and $z_{j}(t)$ can be expressed as:

$$
\rho_{z_{i}, z_{j}}(t, f)=\rho_{z_{i}, z_{i}}(t, f) e^{-j \phi}
$$

Then, knowing that $\rho_{z_{i}, z_{i}}(t, f)$ is real, the phase of cross TFD defined in Eq. (93) is given by:

$$
\bar{\varphi}_{i j}(t, f)=\arg \left[\rho_{z_{i}, z_{j}}(t, f)^{2}\right]=-2 \phi
$$

In the same way the resulting PLV also tends to $1^{10}$. One can conclude that the two methods of time-varying phase estimation lead to the same theoretical behavior of the PLV for the phase locked signals. However, the method defined by Eq. (93) makes it possible to extract additional information on the phase shift of the two signals where the method defined by Eq. (90) is invariant with respect to the phase shift.

\subsection{Illustrative examples}

This simulation uses a forward model ${ }^{11}$ generated from an atlas of neonatal MRI data. The relevant surfaces, a 3D cortex mesh, and the coregistration of the Electrical Geodesics, Inc (EGI) hydrocell caps orientation on the infants head are generated by using the MatLab software, Brainstorm [77, 78]. Once these surfaces are generated, OpenMEEG is then used to generate the lead-field matrix from this model $[79,80]$. A simulator then takes the lead-field matrix and generates virtual sources centered on a single cortical volume. To do so, a virtual source signal is given, along with a cortical volume and dipole orientation for $m=64$ electrodes. The signal is then propagated through the lead-field matrix, and additive noise is included. To establish brain connectivity matrices, the definitions given by Eqs. (90) and (93) are used to calculate the time-varying phase spectra and the corresponding PLV values for all EEG channels.

\footnotetext{
${ }^{10}$ By replacing $\bar{\varphi}_{i j}(t, f)=-2 \phi$ for $\forall(t, f)$ in Eq. (92) the PLV tends to 1.

${ }^{11}$ The forward model is a conduction model describing how signals originating from particular locations within the brain traverse the tissues of the head and are received at the EEG electrodes (see Section 6.1.1).
} 


\subsubsection{Experiment 1}

This experiment was conducted for different values of SNR, in order to asses the robustness of PLV estimation methods. In this experiment, three synthetic signals $(n=3)$; two of them are Gaussian pulse expressed as:

$$
\begin{aligned}
& s_{1}(t)=\exp \left(-40(t-2.8)^{2}\right) \exp (-j 2 \pi(5 t-14)) \\
& s_{2}(t)=\exp \left(-128(t-7)^{2}\right) \exp (-j 2 \pi(6 t-42))
\end{aligned}
$$

and the third is an LFM signal generated as:

$$
s_{3}(t)=\exp \left(-j 2 \pi\left(\frac{1}{2} t^{2}+t\right)\right) \quad t \in[1.8,4.5]
$$

The generated signals are passed through the lead-field matrix and then received on 64 sensors $(m=64)$. As depicted in Fig. 34, the signals are selected in such a way that they do not have cross-terms, so as to avoid the cross-terms in the PLV estimation.

The resulting PLV is represented in Figs. 35, 36 and 37 for received signals with SNR $10 \mathrm{~dB}, 30 \mathrm{~dB}$ and $50 \mathrm{~dB}$ respectively. To describe the cross-channel causality of obtained multichannel EEG signals, we compare for each SNR obtained the PLV by using the standard definition in Eq. (90) and the MTFD based one given by Eq. (93). For the MTFD based PLV definition we used three TFDs; Rihaczek, Wigner-Ville and compact kernel distribution (CKD).

One can observe from Figs. 35, 36 and 37 that the MTFD based PLV definition has better resolution to describe the cross-channel causality and greater noise robustness compared to the PLV estimation based on Eq. (90).

\subsubsection{Experiment 2}

This experiment was conducted for source signals with cross-terms, in order to assess the robustness of PLV estimation using high resolution TFDs. Then, in this experiment, three synthetic signals $(n=3)$; one LFM signal and two 

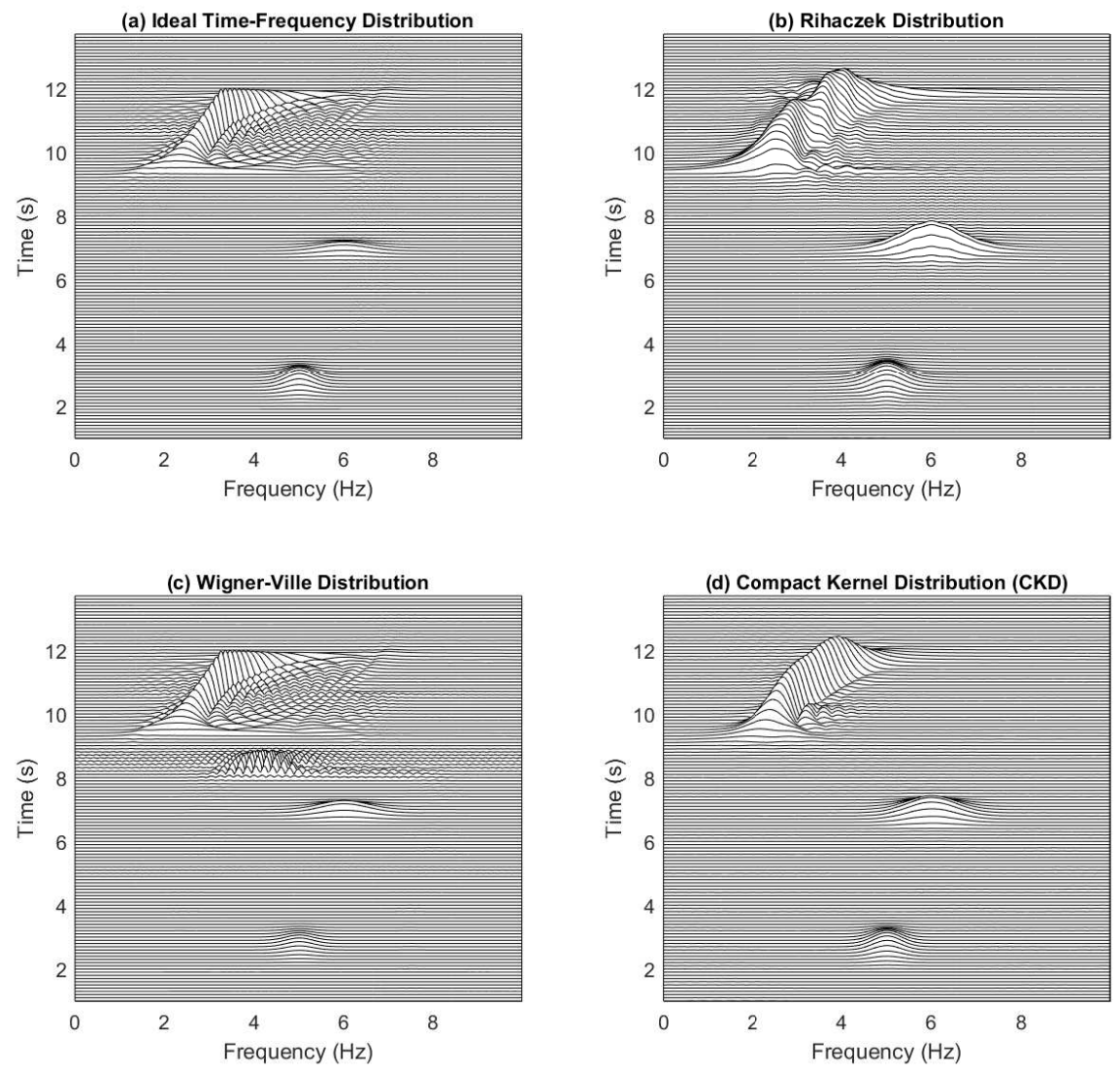

Figure 34: TFDs of received mixed signal for experiment 1: (a) ideal TF representation; (b) Rihaczek distibution; (c) Wigner-Ville distibution; (d) Compact kernel distribution (CKD) with parameters $c=1, D=0.1$ and $E=0.1$.

quadratic FM signal, generated as:

$$
\begin{aligned}
& s_{1}(t)=\exp \left(-j 2 \pi\left(4 t-\frac{25}{32} t^{2}\right)\right), \\
& s_{2}(t)=\exp \left(-j 2 \pi\left(9 t-\frac{1}{64} t^{3}\right)\right), \\
& s_{3}(t)=\exp \left(-j 2 \pi\left(t+\frac{1}{64} t^{3}\right)\right),
\end{aligned}
$$


(a) Standard definition using Rihaczek

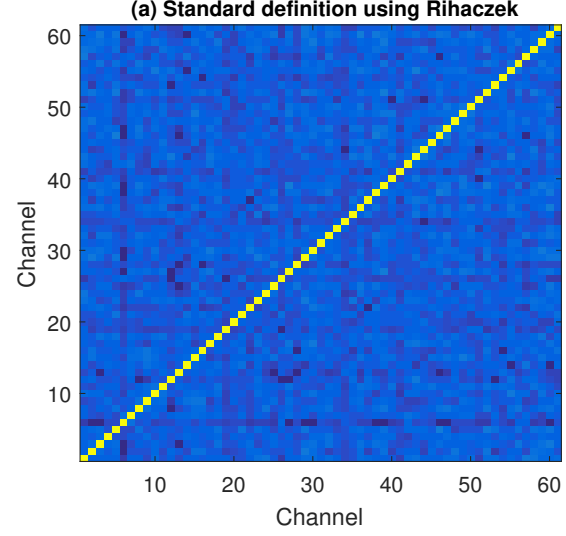

(c) Wigner-Ville Distribution

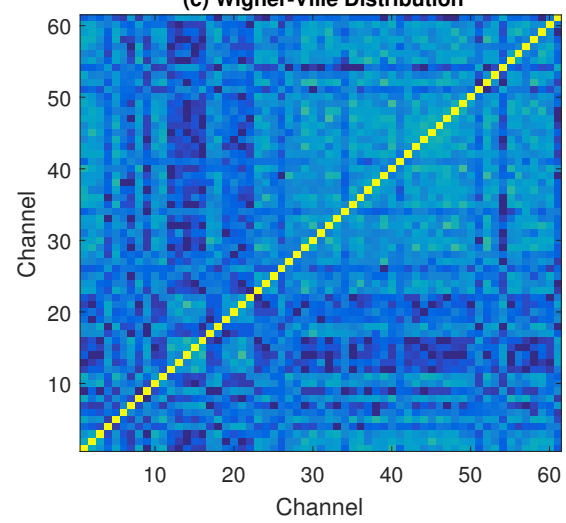

(b) Rihaczek Cross TFD

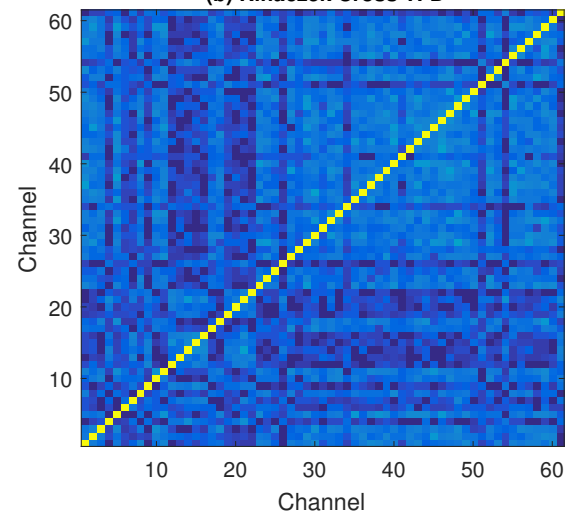

(d) Compact Kernel Distribution (CKD)

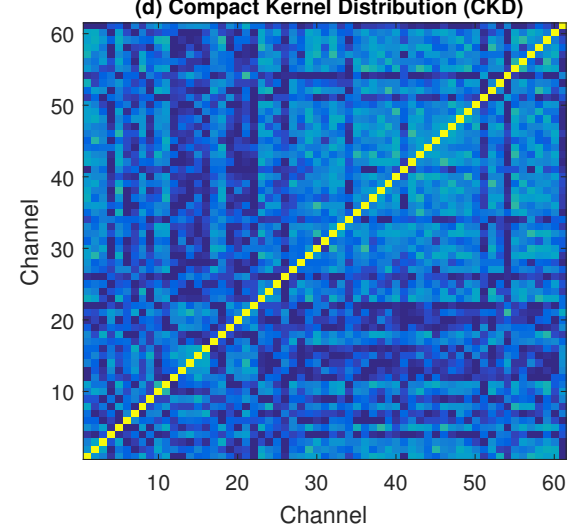

Figure 35: Brain networks representation (newborne): brain connectivity matrices representing the mean PLV matrices $\overline{\mathcal{P}}$ for SNR $=10 \mathrm{~dB}$ using (a) standard definition Eq. (90) based on Rihaczek distribution; (b) Rihaczek distibution with cross MTFD terms; (c) Wigner-Ville distibution with cross MTFD terms; (d) Compact kernel distribution (CKD) with parameters $c=1, D=0.1$ and $E=0.1$ using cross MTFD terms.

and their corresponding instantaneous frequencies (IFs) are given by:

$$
\begin{aligned}
\mathrm{IF}_{s 1}(t) & =\frac{1}{2 \pi} \frac{d \varphi_{s 1}}{d t}(t)=-4+\frac{25}{16} t \\
\mathrm{IF}_{s 2}(t) & =\frac{1}{2 \pi} \frac{d \varphi_{s 2}}{d t}(t)=-9+\frac{3}{64} t^{2} \\
\mathrm{IF}_{s 3}(t) & =\frac{1}{2 \pi} \frac{d \varphi_{s 3}}{d t}(t)=-1-\frac{3}{64} t^{2},
\end{aligned}
$$


(a) Standard definition using Rihaczek

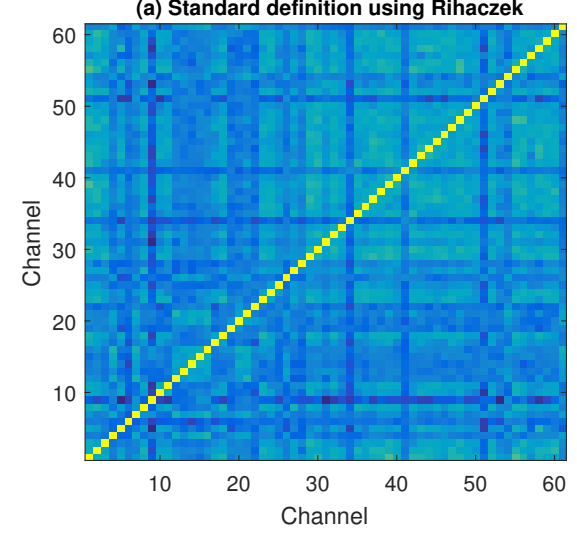

(c) Wigner-Ville Distribution

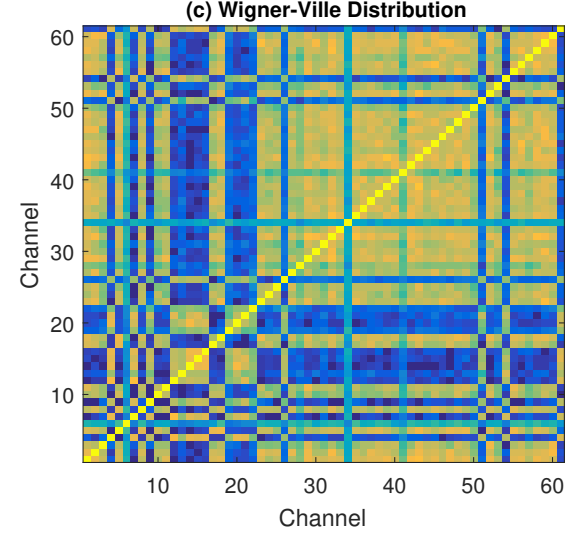

(b) Rihaczek Cross TFD

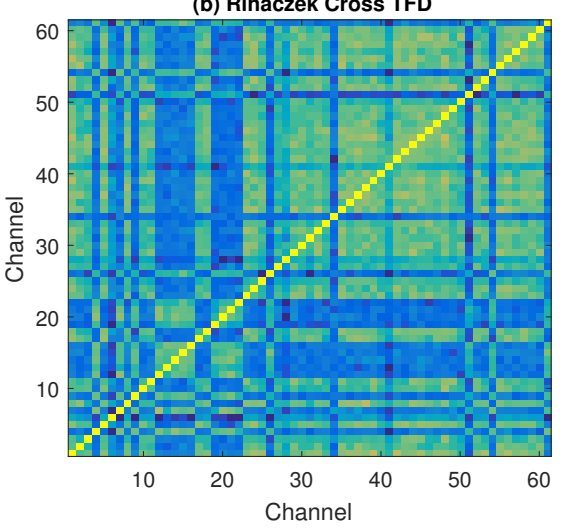

(d) Compact Kernel Distribution (CKD)

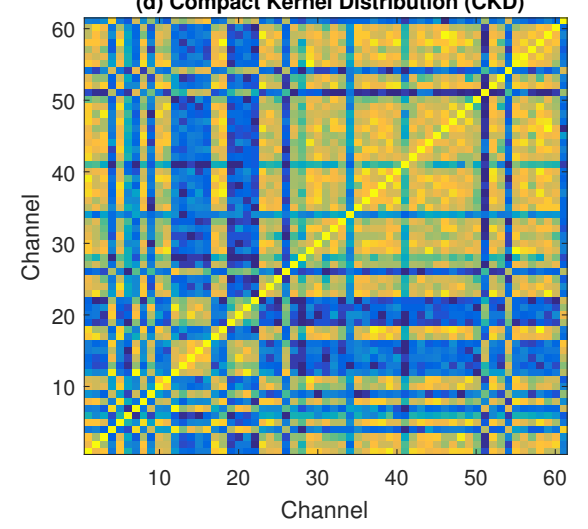

Figure 36: Brain networks representation (newborne): brain connectivity matrices representing the mean PLV matrices $\overline{\mathcal{P}}$ for $\mathrm{SNR}=30 \mathrm{~dB}$ using (a) standard definition Eq. (90) based on Rihaczek distribution; (b) Rihaczek distibution with cross MTFD terms; (c) Wigner-Ville distibution with cross MTFD terms; (d) Compact kernel distribution (CKD) with parameters $c=1, D=0.1$ and $E=0.1$ using cross MTFD terms.

The generated signals are propagated through the lead-field matrix and then received on 61 sensors $(m=61)$. One can observe in Fig. 38(c) the WVD of the received signals, and the distorting effect of inner cross-terms when representing nonlinear FM signals. On the other hand, the CKD represented in Fig. 38(d) demonstrated a better trade-off between resolution and cross-terms suppression.

The computed connectivity matrices based on PLV estimation are not sparse 
(a) Standard definition using Rihaczek

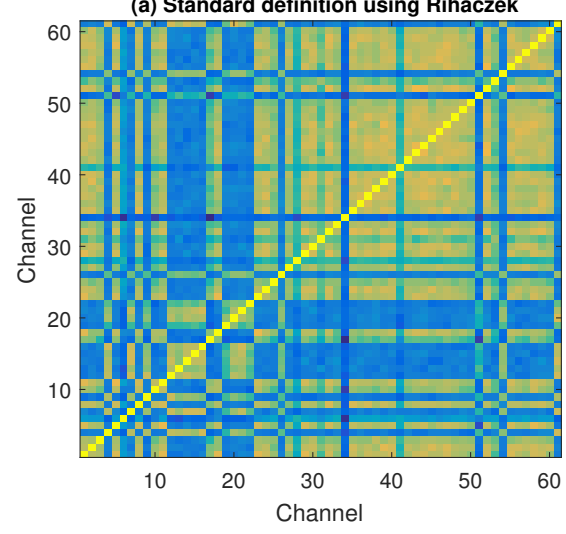

(c) Wigner-Ville Distribution

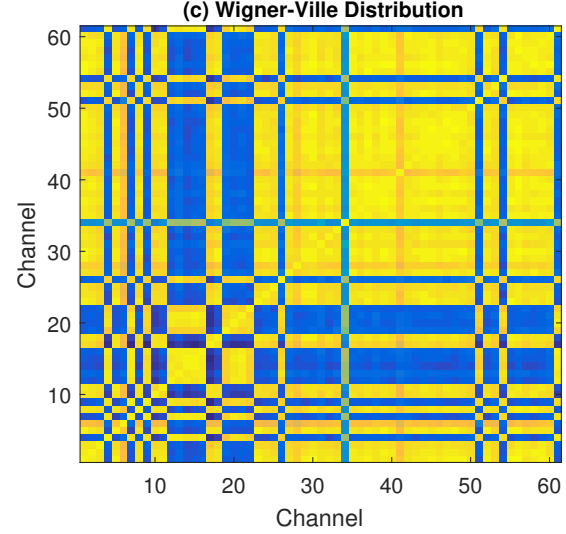

(b) Rihaczek Cross TFD

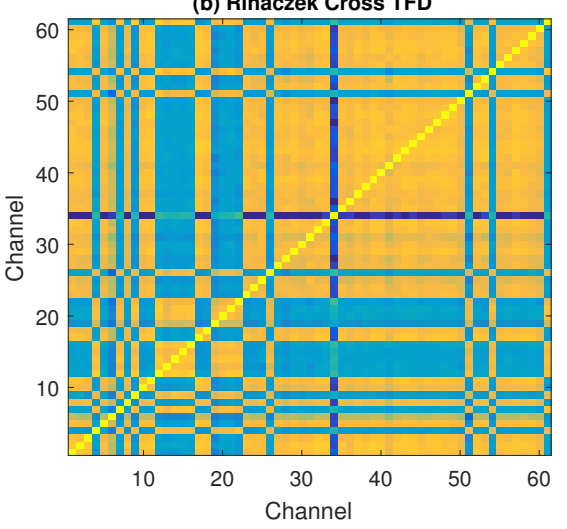

(d) Compact Kernel Distribution (CKD)

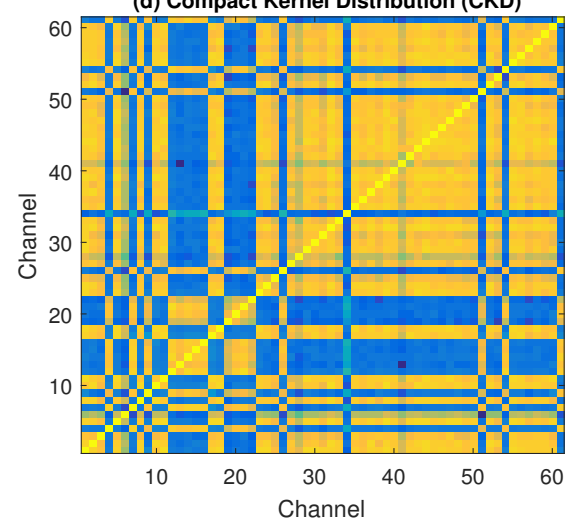

Figure 37: Brain networks representation (newborne): brain connectivity matrices representing the mean PLV matrices $\overline{\mathcal{P}}$ for $\mathrm{SNR}=50 \mathrm{~dB}$ using (a) standard definition Eq. (90) based on Rihaczek distribution; (b) Rihaczek distibution with cross MTFD terms; (c) Wigner-Ville distibution with cross MTFD terms; (d) Compact kernel distribution (CKD) with parameters $c=1, D=0.1$ and $E=0.1$ using cross MTFD terms.

(see e.g. Figs 35, 36 and 37 of Experiment 1), as many spurious connections also appear. Therefore, to concentrate on the most relevant connections, we thresholded the connectivity matrices to keep only the $5 \%$ strongest connections. One can observe from Fig. 39 that connectivity matrices based on PLV estimation using the CKD provide a better resolution to describe the cross-channel causality in presence of cross-terms. Indeed, in this experiment the WVD introduces pseudo-information in the $(t, f)$ domain in the form of inner cross-terms (see 

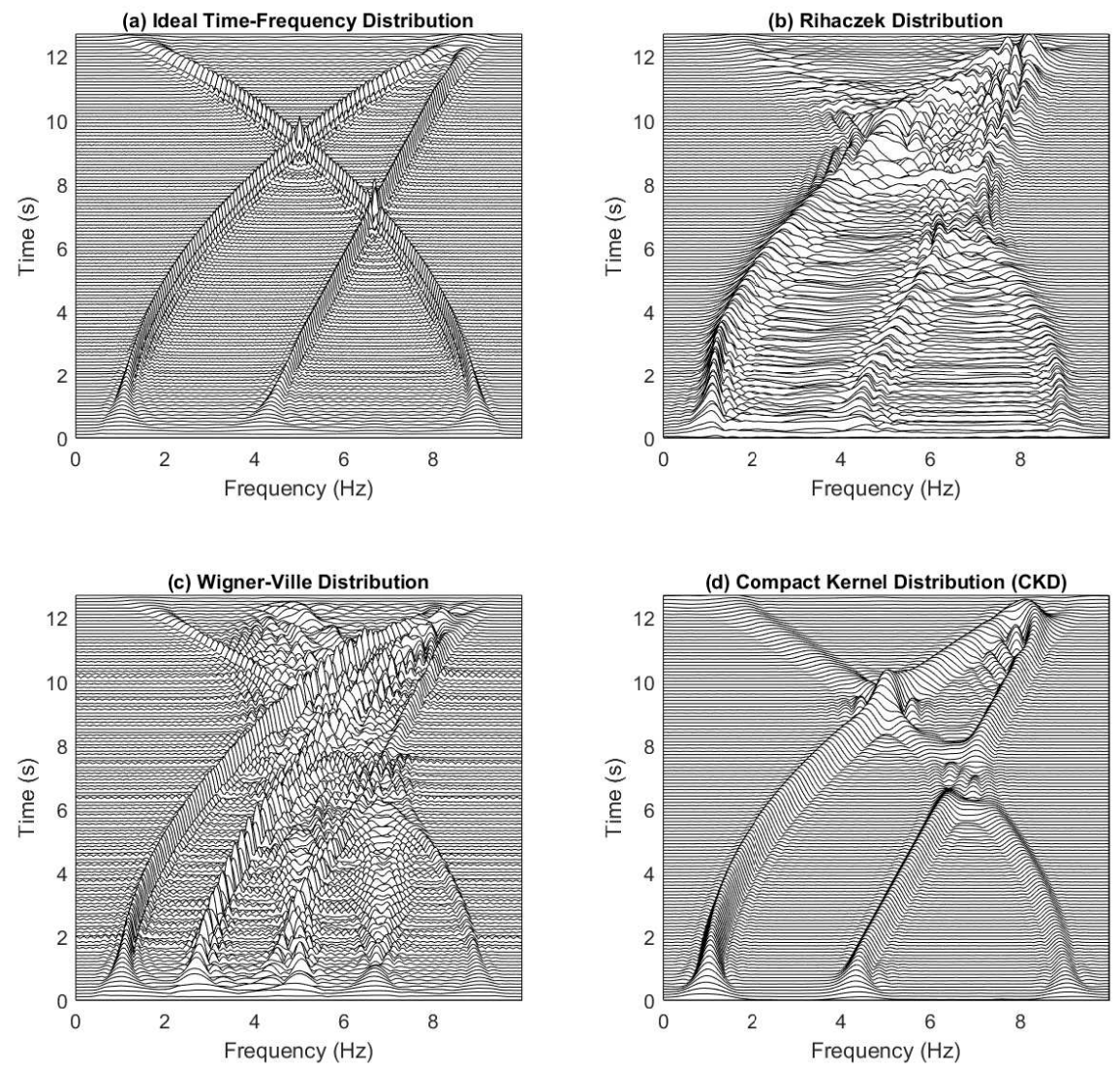

Figure 38: TFDs of received mixed signal for experiment 2: (a) ideal TF representation; (b) Rihaczek distibution; (c) Wigner-Ville distibution; (d) Compact kernel distribution (CKD) with parameters $c=1, D=0.1$ and $E=0.08$.

Fig. 38(c)), which becomes problematic for the PLV estimation. To reduce the cross-terms while keeping a high $(t, f)$ resolution separable kernel methods, presented in Section 2.3.2 can be used. This experiment used the compact kernel distribution (CKD) with parameters $c=1, D=0.1$ and $E=0.08$ (see Fig. 38(d)). One can observe from Figs. 39(c) and 39(d) that the CKD provides a more accurate PLV than that obtained by using the WVD. 

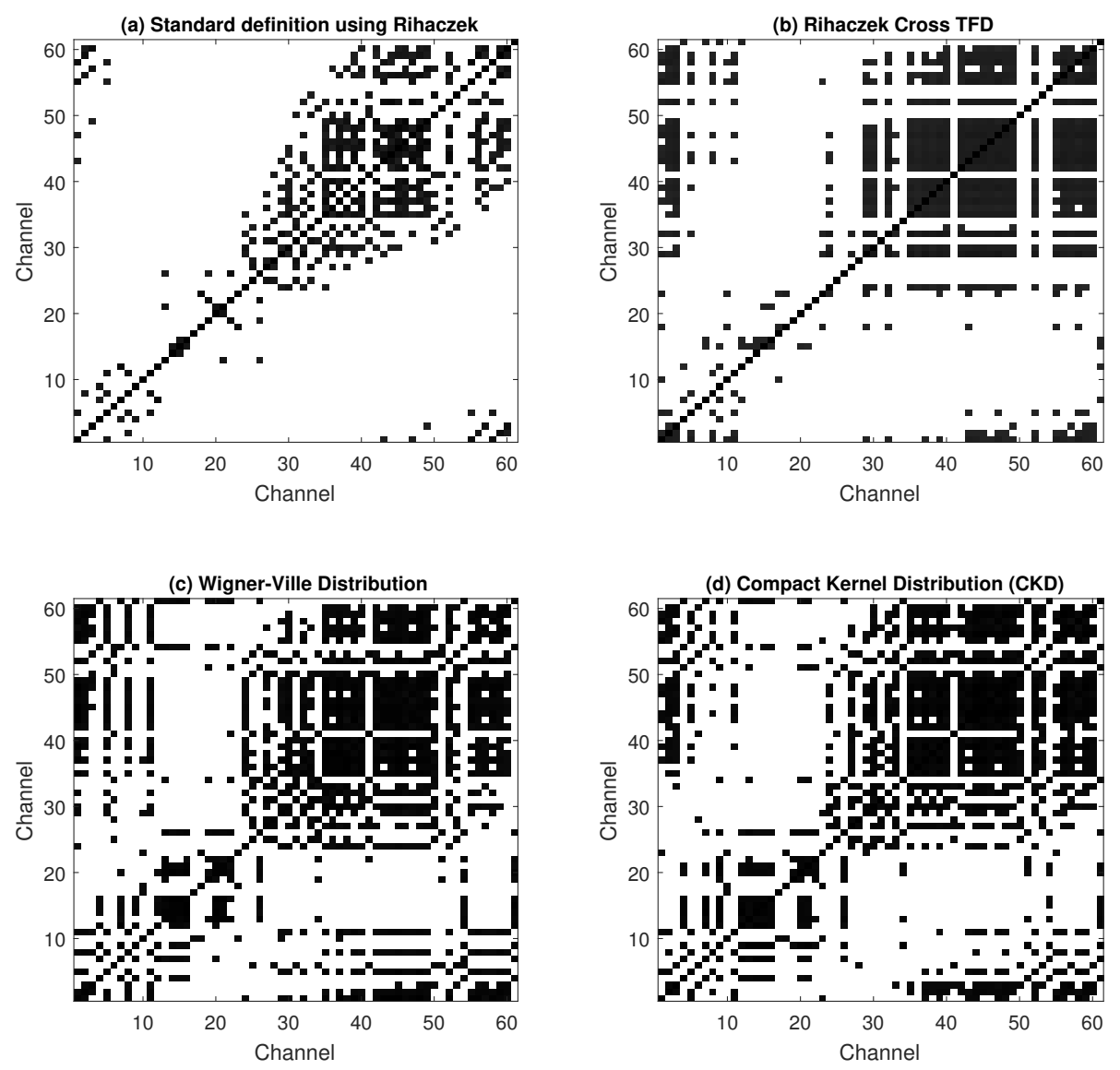

Figure 39: Brain networks representation (newborn): thresholded brain connectivity matrices with keeping only the strongest $5 \%$ of connections using (a) standard definition Eq. (90) based on Rihaczek distribution; (b) Rihaczek distibution with cross MTFD terms; (c) Wigner-Ville distibution with cross MTFD terms; (d) Compact kernel distribution (CKD) with parameters $c=1, D=0.1$ and $E=0.08$ using cross MTFD terms.

\section{Application: multisensor time-frequency analysis of EEG signals}

The relationship between functional brain activity and anatomical sources is important in many clinical situations such as presurgical analysis, and is one of the scientific cutting edge topics of brain research. Electroencephalography (EEG) is a non-invasive method for acquiring neural information that measures electrical potential corresponding to neural activities using sensors 
directly attached to the scalp. Despite its superior temporal resolution, the spatial resolution of EEG is less than other functional brain imaging methods such as fMRI. This is due to the separation between EEG electrodes and the current sources inside the head, i.e. neurons, by several layers with different conductivity profiles [81]. In addition, since various source arrangements inside the brain or cortex can result in a similar potential distribution on the scalp, the visual interpretation of EEG cannot provide an accurate location of neural generators [82].

Some of the well-documented methods are based on the convenient but inaccurate assumption that EEG signals are stationary or at least quasi-stationary $[83,84,85]$. To account for the non-stationary characteristic EEG seizure, a number of time-frequency methods have been proposed [86, 69, 87]. Most studies have focused on only single channel EEG or on single channels at a time when using multichannel EEGs. However, to improve precision and performance, the problem of analyzing multichannel EEGs should be approached more precisely using recently developed TF methods for analyzing multisensor data in the context of array signal processing $[87,88]$. Once an abnormality has been detected and classified in multichannel EEG, the solution of the inverse problem can be used to locate the sources of that abnormality in the brain.

The following extends the MTFD concepts presented earlier to an application dealing with EEG signals enhancement and diagnosis; specifically, we use TFBSS and TF-MUSIC methods for artifacts removal and source localization.

\subsection{Data model}

\subsubsection{Lead Field Matrix}

The EEG forward problem aims at predicting the scalp potentials that result from the hypothetical dipoles, or more generally from current distributions inside the head at any location, orientation and amplitude values [89]. Because most studies of EEG deal with frequencies between $0.1 \mathrm{~Hz}$ and $100 \mathrm{~Hz}$ [90], the forward model can be described by the quasi-static versions of Maxwell's equations [91]. In this condition Poisson's equation gives the potentials at any 
position in a volume conductor due to current source distribution as [92, 93]:

$$
\nabla \cdot(\mu \nabla \vartheta)=\nabla \cdot \mathbf{J}^{\mathbf{s}}, \quad \text { in } \Omega,
$$

where $\nabla$ is a partial differential vector operator, $\Omega$ denotes ohm (unit of the resistance of a conductor), $\mu$ is the electrical conductivity, $\vartheta$ are the electrical potentials and $\mathbf{J}^{\mathbf{s}}$ are the electric current sources. Eq. (94) indicates that for a given configuration of electric sources, the mapping from the electric sources within the head to the scalp recordings on the outside of the scalp can be represented by a lead field matrix [89].

There are two main numerical methods for solving the forward model and obtaining the lead field matrix, namely; boundary element method (BEM) [94, 95] and finite element method (FEM) [96, 97, 98]. Boundary element methods assume that each layer is homogeneous and isotropic in conductivity. Finite element methods can be extended to model anisotropic and inhomogeneous tissues such as skull and white matter. However, It has been shown that when piecewise constant conductivity is used (instead of a spatially varying anisotropic conductivity model), both methods perform similar in terms of the accuracy of the solution [99].

\subsubsection{Formulation}

Let us define $z_{i}(t)$ as the electric field measured at sensor $i$ at time instant $t$. The vector $\mathbf{z}(t)=\left[z_{1}(t), z_{2}(t), \ldots, z_{m}(t)\right]^{T}$ then presents the set of measurements collected by $m$ sensors. Let's assume $n$ current dipole sources generate the electric field. The magnitude of the $i^{\text {th }}$ dipole source movement is $s_{i}(t)$ and the source magnitude vector is defined as: $\mathbf{s}(t)=\left[s_{1}(t), s_{2}(t), \ldots, s_{n}(t)\right]^{T}$. For $m$ sensors and $n$ dipole sources, the relationship between $\mathbf{z}(t)$ and $\mathbf{s}(t)$ can be expressed by Eq.(12) using matrix $\mathbf{A}$ such that $\mathbf{z}(t)=\mathbf{A} \mathbf{s}(t)+\boldsymbol{\eta}(t)$, where $\mathbf{A}$ represents the lead-field matrix of dimension $m \times n$ [89] that includes both the effect of location $\Lambda$ and orientation $\boldsymbol{\Phi}$ of the dipoles as $\mathbf{A}=\boldsymbol{\Lambda} \boldsymbol{\Phi}$. Each column of the lead-field matrix is called a lead field and it defines the current flow for a given sensor through each dipole position [89]. 
Hence, the above model can be used to solve the problem of EEG source localization and artifacts removal using the approaches presented earlier.

\subsection{Application of BSS to EEG artifacts removal}

\subsubsection{Background and motivation}

In this section, the advanced BSS algorithms discussed earlier in the text are applied to the analysis of multichannel EEG signals for artifacts removal.

Artifacts cause a major problem in the implementation of fully automated EEG signal classification systems; e.g. respiratory artifacts look like seizures and can be misinterpreted by the automatic abnormality detection system thus resulting in false alarms. One option is to apply machine learning algorithms to first detect and then reject EEG segments corrupted by artifacts, but this approach results in a loss of EEG data $[2,14]$. Another approach for removing artifacts is to correct EEG signals without discarding any EEG segments. Some artifacts can be corrected by simple frequency domain filtering, e.g. band pass filtering can remove low-frequency movement related artifacts or a notch filter can remove $50 \mathrm{~Hz}$ noise. This approach does not require any reference signals. For more complex cases, when the spectrum of artifacts overlaps with the spectrum of EEG signals, BSS algorithms can be used. In this approach, signals that are corrupted by artifacts are identified either manually or automatically using correlation from a reference signal [100]. The artifact free signal is then synthesized by combining only artifact free components.

Based on the model presented in Section 6.1.1 the instantaneous BSS methods can be considered to tackle the problem of artifacts removal. Therefore, an EEG artifact removal algorithm can be designed by using the BSS algorithm presented in Section 3 with a selected high resolution time-frequency distribution to extract close signal components (see results in Section 6.4). 


\subsection{TF-MUSIC applied to source localization of brain EEG abnormalities}

\subsubsection{Background and motivation}

In this section, the advanced DOA estimation algorithms discussed above are applied to the analysis of multichannel EEG signals.

EEG source localization (ESL) is an important tool used to estimate the intracerebral generators of the potentials observed on the scalp in both clinical and research in cognitive neuroscience. While there is an increasing interest in studying motor evoked potentials (EVP) by means of ESL, epilepsy has been the main focus of the clinical application of ESL in neurology [101]. Similarly, cognitive neuroscience studies have used ESL to investigate temporal information in the event related potentials (ERP), and psychiatry and psychopharmacology has employed ESL to study sources in specific frequency bands [101].

\subsubsection{Source localization of EEG abnormality using TF-MUSIC}

In the context of the brain application, where the aim is to estimate the locations of EEG abnormalities, localization algorithms (such as MUSIC, ESPRIT) exploit the fact that the lead-field matrix $A=\Lambda \Phi$ is orthogonal to the noise subspace of the received covariance matrix [89]. To find the locations of abnormalities, the covariance matrix is estimated from the received multichannel EEG signals, and instead of using steering vectors, in Eq.(71) the eigenvectors are projected on the columns of the lead field matrix $\mathbf{A}$.

More precisely, the TF-MUSIC algorithm, as indicated in Section 4.2.1, starts with calculating the MTFD of the processed EEG data $\boldsymbol{\rho}_{z z}(t, f)$, followed by the average procedure described in Table 1 . To estimate the locations of the sources, the TF-MUSIC algorithm takes advantage of the fact that $\operatorname{span}(\mathbf{A})=\operatorname{span}\left(\mathbf{V}_{s}\right)$ (where $\mathbf{V}_{s}$ is the signal subspace of $\left.\boldsymbol{\rho}_{z z}(t, f)\right)$ and the orthogonality of signal subspace and noise subspace. These can thus be obtained by checking the orthogonality between the lead field matrix and the noise subspace projector. Then, the final TF-MUSIC metric is derived as a measure of 
orthogonality between the noise subspace and the lead-field matrix [102]:

$$
\mathcal{J}=\frac{1}{\lambda_{\min }\left(\mathbf{A}^{H} \mathbf{V} \mathbf{V}^{H} \mathbf{A}, \mathbf{A}^{H} \mathbf{A}\right)}
$$

where $\lambda_{\min }$ is the minimum generalized eigenvalue of the matrix pair $\mathbf{A}^{H} \mathbf{V} \mathbf{V}^{H} \mathbf{A}$ and $\mathbf{A}^{H} \mathbf{A}$, and $\mathbf{V}$ is the noise subspace defined as $\mathbf{V}=$ $\left[\mathbf{v}_{n+1}, \ldots, \mathbf{v}_{m}\right]$ in which $\mathbf{v}_{i}$ is the $i^{\text {th }}$ eigenvector of the averaged MTFD matrix. Calculating this metric over all grid points (source space) then results in a map with a peak at or near the location of the source.

\subsection{Results and discussion}

In this section, numerical and experimental results will be discussed where we apply the concepts of TF BSS and TF-MUSIC on EEG signals for artifacts removal and source localization.

\subsubsection{Experiment: application of BSS to EEG artifacts removal}

In order to illustrate the application of BSS to EEG artifacts removal we propose the following experiment. A 5 seconds segment of clean multichannel EEG was obtained from a publicly available database described in [103]. The obtained EEG segment is recorded using nineteen electrodes, which were placed according to the 10-20 International System. The extracted EEG segment is down-sampled to $100 \mathrm{~Hz}$, and then combined with synthetic multichannel EEG artifacts as depicted in Fig. 40. The TFDs of clean EEG signal and corrupted EEG signal are represented in Figs $42 \mathrm{a}$ and $42 \mathrm{~b}$ respectively. The multichannel contaminated signals are whitened, and their MTFDs are computed for the selection of auto and cross-terms. After that, two independent sources are estimated using the joint diagonalization/joint off-diagonalization algorithm, and artifactual components are identified using a maximum likelihood detector that utilizes an independent template. Finally, clean multichannel EEG is estimated and compared with the original clean EEG, as depicted in Fig. 41 (TFD of the estimated EEG signal is given in Fig. 42c). 


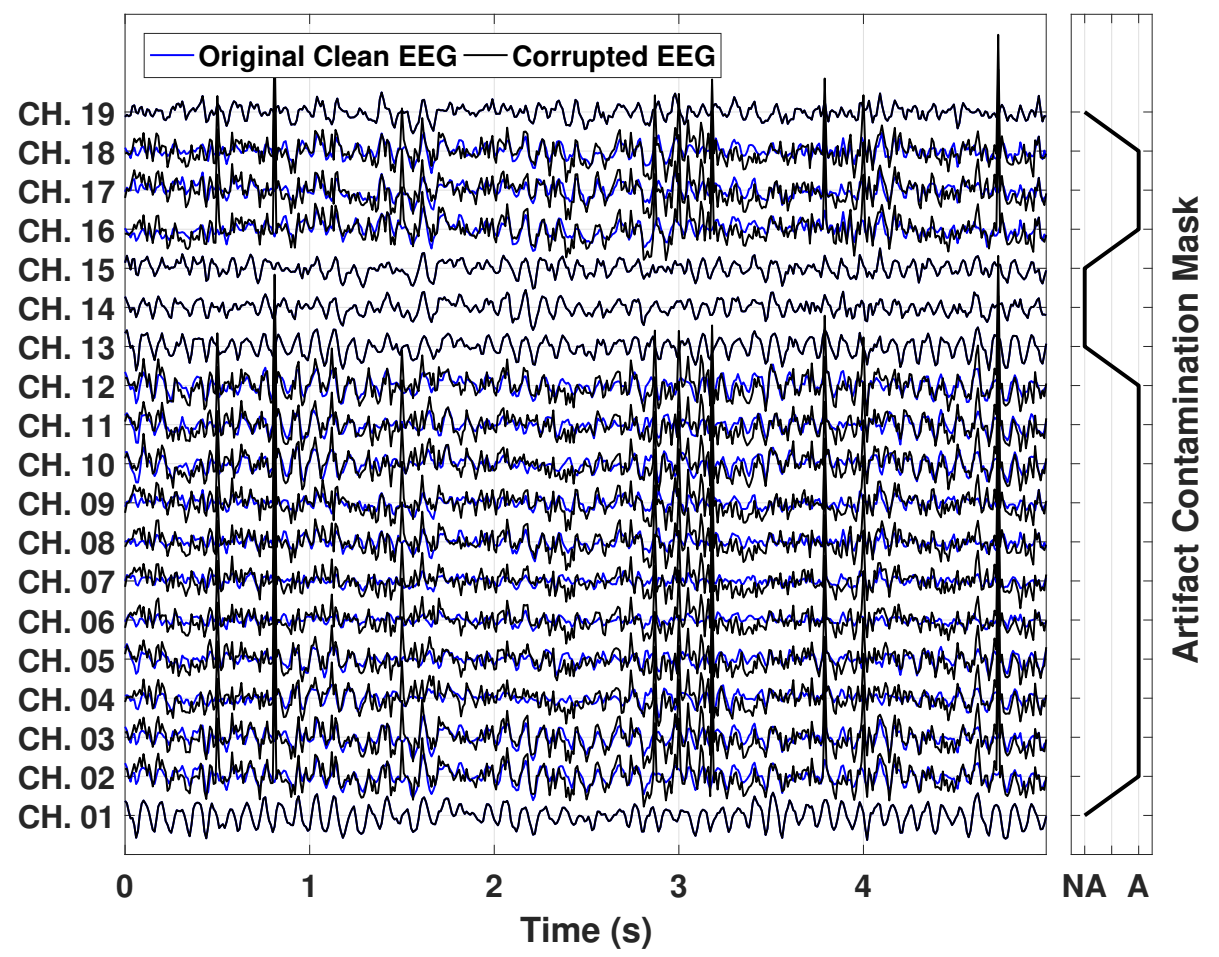

Figure 40: Multichannel clean and corrupted EEG signals (EEG contamination with multichannel artifacts).

\subsubsection{Experiment: TF-MUSIC applied to source localization of EEG signals}

In source localization of EEG signals, generation of a high quality Forward model is key to reproducible results. Small modeling errors may result in significant localization error, as described in [104]. The best case is to have an anatomically accurate physical model of the head available with particular emphasis on the geography and composition of the surfaces of the scalp, outer skull, inner skull and cortex, and accurately localized electrode locations. This is generally best done using a brain magnetic resonance image and either manual or automated segmentation of the surfaces.

In this simulation the MatLaB software, Brainstorm [77, 78], was used to generate a forward model from an atlas of neonatal MRI data. The relevant surfaces, a 3D cortex mesh, and the coregistration of the EGI hydrocell caps 
- Original Clean EEG — Estimated EEG

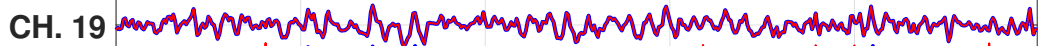

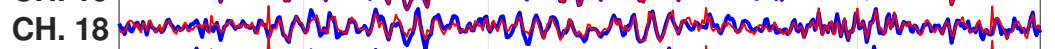

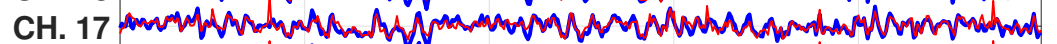

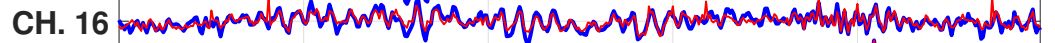

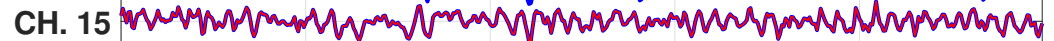

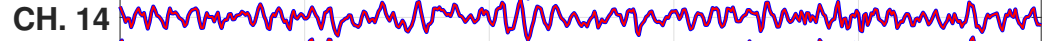
CH. $13 \mathrm{AWH}$.

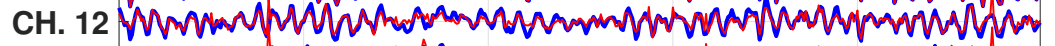

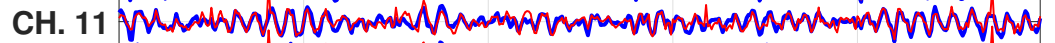

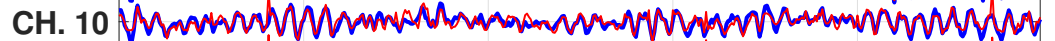

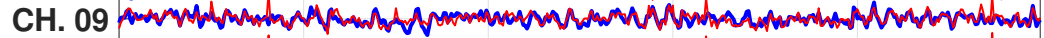

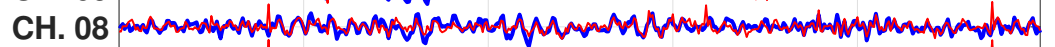

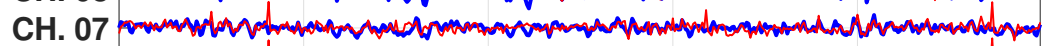

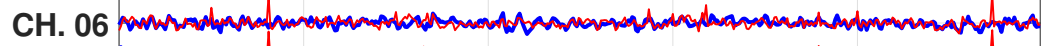

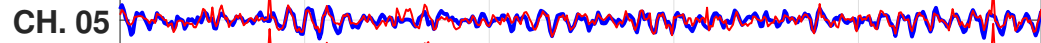

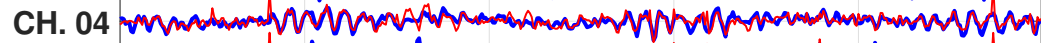

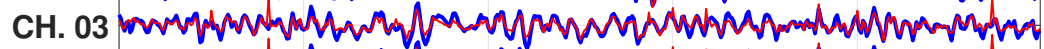

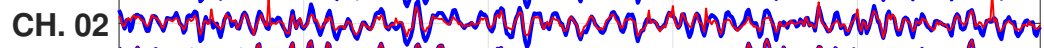

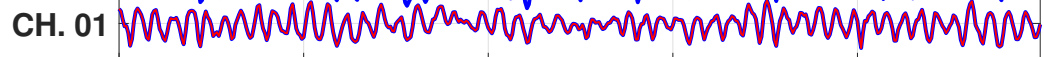

$\begin{array}{lllll}0 & 2 & & & \\ & & & \end{array} \quad$ NA A

Figure 41: Multichannel clean EEG signals and theirs estimates using the TF BSS algorithm (artifacts detection and removal using TF BSS algorithm where the signal in red represents the estimated EEG signal by TF-BSS algorithm).

orientation on the infants head are generated by using the Brainstorm toolbox. Once these surfaces were generated, OpenMEEG was then used to generate the lead-field matrix from this model $[79,80]$. A simulation has been developed which takes the lead-field matrix and generates virtual sources centered on a single cortical volume. To do so a virtual source signal, in this demonstration a Gaussian pulse, is given, along with a cortical volume and dipole orientation for 64 electrodes. The signal is then propagated through the lead-field matrix, and additive noise is included.

MUSIC and TF-MUSIC algorithms are applied to estimate the source locations for several noise realisations with $\mathrm{SNR}=3 \mathrm{~dB}$. Fig. 43 shows the location of three virtual signals on the cortical volume, highlighted in black, and the esti- 


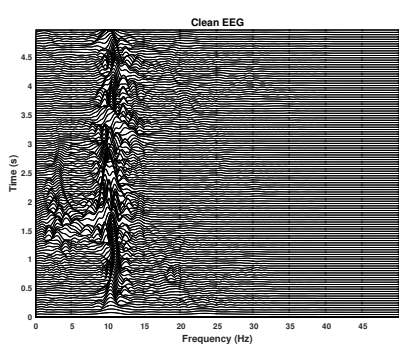

(a) Clean EEG

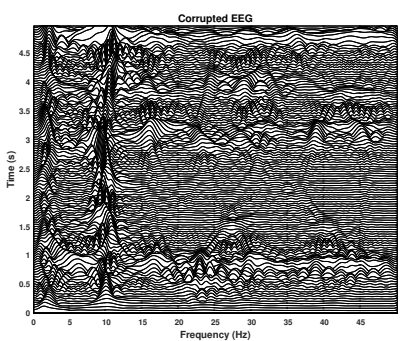

(b) Corrupted EEG

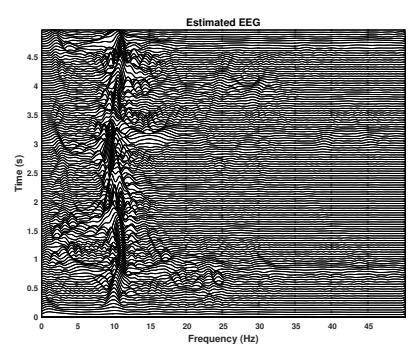

(c) Estimated EEG

Figure 42: TFDs of EEG signal: a) Clean EEG signal b) EEG signal corrupted by artifact c) Estimated EEG signal (by using BSS algorithm).

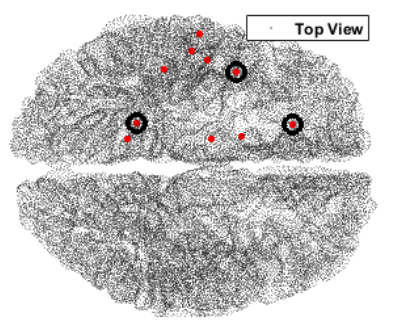

\section{Conventional MUSIC}
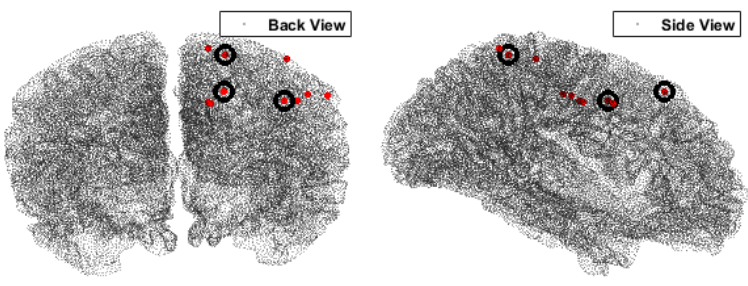

Time-Frequency MUSIC
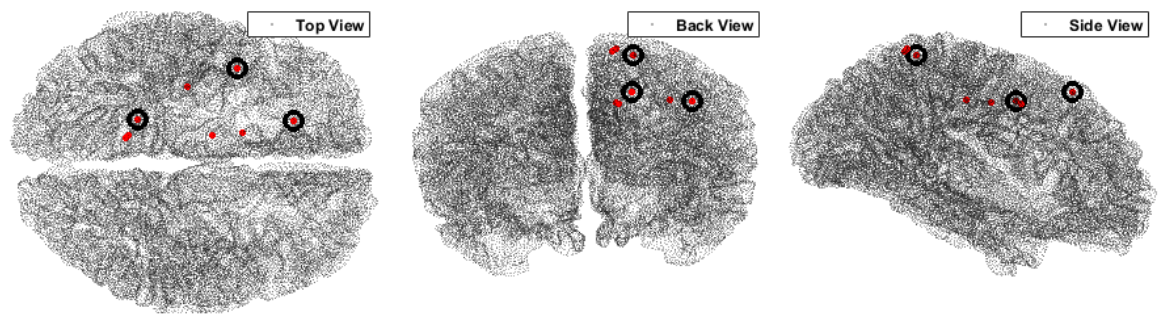

Figure 43: Localization of abnormality sources from 64 EEG channels using TF-MUSIC (newborn).

mated locations highlighted in red by using MUSIC and TF-MUSIC algorithms for 1000 simulation runs. The results indicate that TF-MUSIC outperforms its time-domain counterpart in terms of source localization accuracy. 


\section{Summary and conclusions}

This study presents a rigorous tutorial review of multisensor high-resolution time-frequency distributions (MTFDs) and a discussion of their area of application, with a special EEG feasibility case study. MTFDs effectively combine time-frequency analysis and array signal processing and are formulated by:

- Extending the principles of single-variable TFDs to multisensor/multichannel TFDs,

- Extending conventional stationary array processing to the non-stationary case using time-frequency methods.

- Using principles of high-resolution TFD design [105].

To demonstrate the benefits of MTFDs, this study considered several applications including source localization based on direction of arrival (DOA) estimation and blind source separation (BSS) of non-stationary sources. One key aspect of this study is to illustrate clearly that, in these two applications, the incorporation of time-frequency (TF) concepts enhances the attributes of multisensor receivers. The key reason for this enhancement is the possible exploitation of the signal power localization properties in the $(t, f)$ domain by expressing the multisensor data in terms of the source TF signatures. By doing so, enhancement of signal to noise ratio (SNR) is obtained prior to performing subspace decomposition for source localization and source separation. Analytical reasoning also indicates that distinction in the TF signatures of closely spaced sources allows reducing the number of sources in the field to a single source. This enables processing more sources than sensors and further enhances the performance of the techniques for DOA estimation and BSS.

In the second part of the study, time-frequency DOA estimation approaches (such as the TF-MUSIC and the TF-ESPRIT algorithms) are discussed and compared with the conventional time domain DOA estimation approaches in the context of localizing the sources of EEG abnormalities. The findings indicate that compared to traditional DOA estimation algorithms, when properly 
used, MTFDs perform better for the localization of the sources of EEG abnormalities. It is because; 1) EEG signals are non-stationary in nature, 2) TF DOA estimation algorithms exploit the signal signature localization properties of MTFDs through the selection of the high energy $(t, f)$ points.

However, this detailed presentation of MTFDs indicates that there is a lot of potential for extracting additional useful information from multisensor/multichannel data sets in a wide range of fields, such as feature extraction for change detection in biomedical signal processing or to detect early structural problem in structural health monitoring. Also, key methodologies have been provided and illustrated on selected examples with applications to audio and EEG signals. The MTFSP toolbox [5] needed to reproduce the results presented in this paper is provided as Supplementary Material and outlined in a companion paper [5].

\section{Acknowledgements}

This research was funded by Qatar Foundation grants NPRP 6-885-2-364 and NPRP 6-680-2-282. The authors thank Mohamed Fathi Al-Sad for his valuable technical help. The first author thanks Prof. Paul Colditz for providing the EEG data and the lead-field matrix as part of the grant NPRP 6-885-2-364.

\section{Appendix A. Definitions, Terminology and Notations}

Symbols frequently used in this paper are listed below in alphabetical order. The meaning in the list below should be assumed unless the symbol is otherwise defined in context.

$$
\begin{aligned}
\mathcal{A}_{z}(\nu, \tau) & \text { symmetrical ambiguity function (SAF) of } z(t) \\
& =\int_{-\infty}^{\infty} z\left(t+\frac{\tau}{2}\right) z^{*}\left(t-\frac{\tau}{2}\right) e^{-j 2 \pi \nu t} d t \\
f & \text { frequency } \\
\underset{t \rightarrow f}{\mathcal{F}}\{x(t)\} & \text { Fourier transform (FT) of } x(t), \text { to } f \text { domain }
\end{aligned}
$$


$\underset{f \rightarrow t}{\mathcal{F}^{-1}}\{X(f)\}$ inverse Fourier transform (IFT) of $X(f)$, back to $t$ domain

$G(t, \tau)$ time-lag kernel $=\int_{-\infty}^{\infty} g(\nu, \tau) e^{j 2 \pi \nu t} d \nu$

$g(\nu, \tau) \quad$ Doppler-lag kernel

$\mathbf{I}_{n} \quad n \times n$ identity matrix

$K_{z}(t, \tau)$ instantaneous auto-correlation function (IAF) of $z(t)$

$=z\left(t+\frac{\tau}{2}\right) z^{*}\left(t-\frac{\tau}{2}\right)$

$\mathbf{K}_{z z}(t, f)$ represents spatial instantaneous autocorrelation function (SIAF)

$T$ duration of signal

$t$ time

$w(t) \quad$ window function

$W_{z}(t, f) \quad$ Wigner-Ville distribution (WVD) of $z(t)$

$=\int_{-\infty}^{\infty} z\left(t+\frac{\tau}{2}\right) z^{*}\left(t-\frac{\tau}{2}\right) e^{-j 2 \pi f \tau} d \tau$

$\gamma(t, f)$ time-frequency kernel $=\int_{-\infty}^{\infty} G(t, \tau) e^{-j 2 \pi f \tau} d \tau$

$\eta(t)$ additive noise with mean $\mu_{\eta}$ and variance $\sigma_{\eta}^{2}$

$\nu \quad$ Doppler (frequency shift)

$\rho_{z}(t, f)$ quadratic time-frequency distribution (TFD) of signal $z(t)$

$=\int_{-\infty}^{\infty} \int_{-\infty}^{\infty} \int_{-\infty}^{\infty} e^{j 2 \pi \nu(t-u)} g(\nu, \tau) z\left(u+\frac{\tau}{2}\right) z^{*}\left(u-\frac{\tau}{2}\right) e^{-j 2 \pi f \tau} d \nu d u d \tau$

$\boldsymbol{\rho}_{z z}(t, f)$ represents multisensor time-frequency distributions

$\tau$ lag (delay, time shift)

$*$ convolution in time

$\underset{t f}{* *} 2 \mathrm{D}$ convolution in both time and frequency

$\triangleq$ equal by definition, or defined as

$\propto$ proportional to

$\int_{\mathbb{R}} \equiv \int_{-\infty}^{\infty}$

$\int_{\mathbb{R}^{2}} \equiv \int_{-\infty}^{\infty} \int_{-\infty}^{\infty}$ 
Appendix B. Supplementary material for MTFSP Matlab Package

The additional material, MTFSP MATLAB package [5], is provided by the authors to assist the reader to better understand the concepts introduced in this paper. The MatLaB functions and scripts listed below are used to reproduce some of the supporting figures of this papers.

\section{\begin{tabular}{l|l} 
Supplementary material & Figures
\end{tabular}}

Section 2 : Extension of single sensor TFDs to multisensor TFDs

\begin{tabular}{|l|l|}
\hline Demo_MTFD_example & Figs. 7 and 8
\end{tabular}

Section 3 : Blind source separation (BSS)

\begin{tabular}{|l|l|}
\hline \hline Demo_BSS_instantaneous_mix & Figs. 11 and 12. \\
\hline Demo_BSS_convolutive_mix & Figs. 13 and 14 \\
\hline Demo_BSS_convolutive_sound & Figs. 15,16 and 17 \\
\hline Demo_MultiComponent_Signal & Fig. 18 \\
\hline Demo_UBSS_instantaneous_mix_1 & Fig. 21 \\
\hline Demo_UBSS_instantaneous_mix_2 & Figs. 22 and 23 \\
\hline \multicolumn{2}{|c|}{ Section 4 : Direction of arrival estimation using MTFDs } \\
\hline \hline Demo_DOA_Results & Figs. $27,28,29$ and 30 \\
\hline Demo_DOA_SignalSubspace & Figs. 31 and 32 \\
\hline Demo_DOA_SpatialResolution & Fig. 33 \\
\hline \multicolumn{2}{|c|}{ Section 5 : Cross channel causality analysis } \\
\hline \hline Demo_Causality & Figs. $34,35,36,37,38$ and 39 \\
\hline Section 6 : Application: multisensor time-frequency analysis of EEG signals \\
\hline \hline Demo_BSS_EEG & Figs. 40,41 and 42 \\
\hline Demo_DOA_EEG & Fig. 43 \\
\hline
\end{tabular}




\section{Index}

ACS, Automated component separation, $1,7,26$

UBSS, Underdetermined blind source separation, 30, 42-44, 46, 52,

Artifacts, 1, 11, 27, 28, 82-86 53,55

BSS, Blind source separation, 1, 26, WVD, Wigner-Ville distribution, 11, 28-30, 32, 35, 38, 42, 49, 51, $26,35,40,41,49,61,79-81$ 83-85, 87, 89

DOA, Direction of arrival, 1, 7, 9, 15, $24,55-57,59-62,64,67,84$, 89

EEG, Electroencephalography, 1, 6-8, $27,28,68,74,75,81-86,88$

ESPRIT, 58, 60, 61, 67, 84

Hilbert transform, 10, 70

JD, Joint diagonalization, 30, 33-35

JOD, Joint off-diagonalization, 30, 3335

MTFD, Multichannel time-frequency distribution, 8, 12, 19, 22, 30, $31,37,59,60,63,71,75,82$, $85,86,88$

MUSIC, 57-59, 61, 65, 67, 84, 88

TFD, Time-frequency distribution, 7, $11,13,19,22-25,32,33,45-$ $49,60,63,70-73,86,89$ 


\section{References}

[1] B. Boashash (Ed.), Time-frequency signal analysis and processing, 2nd Edition, Academic Press, Oxford, 2016.

[2] B. Boashash, G. Azemi, J. M. O'Toole, Time-frequency processing of nonstationary signals: Advanced TFD design to aid diagnosis with highlights from medical applications, IEEE Signal Processing Magazine 30 (6) (2013) 108-119. doi:10.1109/MSP.2013.2265914.

[3] A. Belouchrani, M. G. Amin, N. Thirion-Moreau, Y. D. Zhang, Source separation and localization using time-frequency distributions: An overview, IEEE Signal Processing Magazine 30 (6) (2013) 97-107. doi:10.1109/MSP.2013.2265315.

[4] A. Aïssa-El-Bey, N. Linh-Trung, K. Abed-Meraim, A. Belouchrani, Y. Grenier, Underdetermined blind separation of nondisjoint sources in the time-frequency domain, IEEE Transactions on Signal Processing 55 (3) (2007) 897-907. doi:10.1109/TSP.2006.888877.

[5] B. Boashash, A. Aïssa-El-Bey, Multisensor time-frequency signal processing software MATLAB package: An analysis tool for multichannel nonstationary data, SoftwareX (in press) (2017) .

[6] D. E. Carlson, J. T. Vogelstein, Q. Wu, W. Lian, M. Zhou, C. R. Stoetzner, D. Kipke, D. Weber, D. B. Dunson, L. Carin, Multichannel electrophysiological spike sorting via joint dictionary learning and mixture modeling, IEEE Transactions on Biomedical Engineering 61 (1) (2014) 41-54. doi:10.1109/TBME.2013.2275751.

[7] C. Weiss, A. M. Zoubir, A sparse regularization technique for source localization with non-uniform sensor gain, in: IEEE 8th Sensor Array and Multichannel Signal Processing Workshop (SAM), 2014, pp. 93-96. doi:10.1109/SAM.2014.6882346. 
[8] Y. Fadlallah, A. Aïssa-El-Bey, K. Amis, D. Pastor, R. Pyndiah, New iterative detector of MIMO transmission using sparse decomposition, IEEE Transactions on Vehicular Technology 64 (8) (2015) 3458-3464. doi:10.1109/TVT.2014.2360687.

[9] A. Ä̈ssa-El-Bey, D. Pastor, S. M. A. Sbaï, Y. Fadlallah, Sparsity-based recovery of finite alphabet solutions to underdetermined linear systems, IEEE Transactions on Information Theory 61 (4) (2015) 2008-2018. doi:10.1109/TIT.2015.2399914.

[10] P. Langley, S. King, K. Wang, D. Zheng, R. Giovannini, M. Bojarnejad, A. Murray, Estimation of missing data in multi-channel physiological timeseries by average substitution with timing from a reference channel, in: Computing in Cardiology, 2010, pp. 309-312.

[11] M. R. Mowla, S.-C. Ng, M. S. Zilany, R. Paramesran, Artifacts-matched blind source separation and wavelet transform for multichannel EEG denoising, Biomedical Signal Processing and Control 22 (2015) 111-118. doi:10.1016/j.bspc.2015.06.009.

[12] I. Bayram, A multichannel audio denoising formulation based on spectral sparsity, IEEE/ACM Transactions on Audio, Speech, and Language Processing 23 (12) (2015) 2272-2285. doi:10.1109/TASLP.2015.2479042.

[13] S. Mirzaei, H. V. Hamme, Y. Norouzi, Blind audio source counting and separation of anechoic mixtures using the multichannel complex NMF framework, Signal Processing 115 (2015) 27-37. doi:10.1016/j.sigpro.2015.03.006.

[14] B. Boashash, S. Ouelha, Automatic signal abnormality detection using time-frequency features and machine learning: A newborn EEG seizure case study, Knowledge-Based Systems 106 (2016) 38-50. doi:10.1016/j.knosys.2016.05.027. 
[15] B. Boashash, G. Azemi, A review of time-frequency matched filter design with application to seizure detection in multichannel newborn EEG, Digital Signal Processing 28 (2014) 28-38. doi:10.1016/j.dsp.2014.02.007.

[16] P. Shen, S. Liu, W. Zhou, F. Lin, A. Lam, H. Sung, W. Chen, J. Lin, M. Chiu, M. Pan, J. Kao, F. Lai, A physiology-based seizure detection system for multichannel EEG, PLoS ONE 8 (6), e65862. doi:10.1371/journal.pone.0065862.

[17] K. Fu, J. Qu, Y. Chai, T. Zou, Hilbert marginal spectrum analysis for automatic seizure detection in EEG signals, Biomedical Signal Processing and Control 18 (2015) 179-185. doi:10.1016/j.bspc.2015.01.002.

[18] M. Varanini, G. Tartarisco, R. Balocchi, A. Macerata, G. Pioggia, L. Billeci, A new method for QRS complex detection in multichannel ECG: Application to self-monitoring of fetal health, Computers in Biology and Medicine (2016) -doi:10.1016/j.compbiomed.2016.04.008.

[19] V. Ionescu, Fetal ECG extraction from multichannel abdominal ECG recordings for health monitoring during labor, Procedia Technology 22 (2016) 682-689. doi:10.1016/j.protcy.2016.01.143.

[20] X. Wang, A. Mortazawi, Medium wave energy scavenging for wireless structural health monitoring sensors, IEEE Transactions on Microwave Theory and Techniques 62 (4) (2014) 1067-1073. doi:10.1109/TMTT.2014.2304918.

[21] Z. Zou, Y. Bao, H. Li, B. F. Spencer, J. Ou, Embedding compressive sensing-based data loss recovery algorithm into wireless smart sensors for structural health monitoring, IEEE Sensors Journal 15 (2) (2015) 797-808. doi:10.1109/JSEN.2014.2353032.

[22] Z. Herrasti, I. Val, I. Gabilondo, J. Berganzo, A. Arriola, F. Martínez, Wireless sensor nodes for generic signal conditioning: Application to struc- 
tural health monitoring of wind turbines, Sensors and Actuators A: Physical 247 (2016) 604-613. doi:10.1016/j.sna.2016.06.027.

[23] D. Liang, S. Yuan, Structural health monitoring system based on multiagent coordination and fusion for large structure, Advances in Engineering Software 86 (2015) 1-12. doi:10.1016/j.advengsoft.2015.03.008.

[24] W.-T. Sung, H.-Y. Chung, A distributed energy monitoring network system based on data fusion via improved PSO, Measurement 55 (2014) 362-374. doi:10.1016/j.measurement.2014.05.007.

[25] F. Doyle, M.-J. R. Duarte, J. Cosgrove, Design of an embedded sensor network for application in energy monitoring of commercial and industrial facilities, Energy Procedia 83 (2015) 504-514. doi:10.1016/j.egypro.2015.12.170.

[26] A. Omidvarnia, G. Azemi, B. Boashash, J. M. O'Toole, P. B. Colditz, S. Vanhatalo, Measuring time-varying information flow in scalp EEG signals: Orthogonalized partial directed coherence, IEEE Transactions on Biomedical Engineering 61 (3) (2014) 680-693. doi:10.1109/TBME.2013.2286394.

[27] J. Lerga, V. Sucic, B. Boashash, An efficient algorithm for instantaneous frequency estimation of nonstationary multicomponent signals in low SNR, EURASIP Journal on Advances in Signal Processing 2011 (1) (2011) 725189. doi:10.1155/2011/725189.

[28] M. G. Amin, Y. Zhang, Direction finding based on spatial time-frequency distribution matrices, Digital Signal Processing 10 (4) (2000) 325-339. doi:10.1006/dspr.2000.0374.

[29] Y. Zhang, M. G. Amin, Spatial averaging of time-frequency distributions for signal recovery in uniform linear arrays, IEEE Transactions on Signal Processing 48 (10) (2000) 2892-2902. doi:10.1109/78.869043. 
[30] L. Stankovic, A method for time-frequency analysis, IEEE Transactions on Signal Processing 42 (1) (1994) 225-229. doi:10.1109/78.258146.

[31] M. Abed, A. Belouchrani, M. Cheriet, B. Boashash, Time-frequency distributions based on compact support kernels: Properties and performance evaluation, IEEE Transactions on Signal Processing 60 (6) (2012) 28142827. doi:10.1109/TSP.2012.2190065.

[32] G. M. Kautz, M. D. Zoltowski, Beamspace DOA estimation featuring multirate eigenvector processing, IEEE Transactions on Signal Processing 44 (7) (1996) 1765-1778. doi:10.1109/78.510623.

[33] A. Hyvarinen, J. Karhunen, E. Oja, Independent component analysis, 1st Edition, Adaptive and learning systems for signal processing, communications, and control, John Wiley, New York, Chichester, Weinheim, 2001.

[34] L. Parra, C. Spence, Convolutive blind separation of non-stationary sources, IEEE Transactions on Speech and Audio Processing 8 (3) (2000) 320-327. doi:10.1109/89.841214.

[35] A. Belouchrani, K. Abed-Meraim, M. G. Amin, A. M. Zoubir, Blind separation of nonstationary sources, IEEE Signal Processing Letters 11 (7) (2004) 605-608. doi:10.1109/LSP.2004.830119.

[36] L. Giulieri, H. Ghennioui, N. Thirion-Moreau, E. Moreau, Nonorthogonal joint diagonalization of spatial quadratic time-frequency matrices for source separation, IEEE Signal Processing Letters 12 (5) (2005) 415-418. doi:10.1109/LSP.2005.843760.

[37] A. Yeredor, Non-orthogonal joint diagonalization in the least-squares sense with application in blind source separation, IEEE Transactions on Signal Processing 50 (7) (2002) 1545-1553. doi:10.1109/TSP.2002.1011195.

[38] G. Chabriel, M. Kleinsteuber, E. Moreau, H. Shen, P. Tichavsky, A. Yeredor, Joint matrices decompositions and blind source separation: A survey 
of methods, identification, and applications, IEEE Signal Processing Magazine 31 (3) (2014) 34-43. doi:10.1109/MSP.2014.2298045.

[39] A. R. Leyman, Z. M. Kamran, K. Abed-Meraim, Higher-order time frequency-based blind source separation technique, IEEE Signal Processing Letters 7 (7) (2000) 193-196. doi:10.1109/97.847366.

[40] N. Linh-Trung, A. Belouchrani, K. Abed-Meraim, B. Boashash, Separating more sources than sensors using time-frequency distributions, EURASIP Journal on Advances in Signal Processing 2005 (17) (2005) 845079. doi:10.1155/ASP.2005.2828.

[41] M. Castella, A. Chevreuil, J.-C. Pesquet, Chapter 8 - convolutive mixtures, in: P. Comon, C. Jutten (Eds.), Handbook of Blind Source Separation, Academic Press, Oxford, 2010, pp. 281-324. doi:10.1016/B978-012-374726-6.00013-8.

[42] A. Belouchrani, M. G. Amin, K. Abed-Meraim, Direction finding in correlated noise fields based on joint block-diagonalization of spatio-temporal correlation matrices, IEEE Signal Processing Letters 4 (9) (1997) 266-268. doi:10.1109/97.623045.

[43] H. Bousbia-Salah, A. Belouchrani, K. Abed-Meraim, Jacobi-like algorithm for blind signal separation of convolutive mixtures, Electronics Letters 37 (16) (2001) 1049-1050. doi:10.1049/el:20010698.

[44] K. Abed-Meraim, W. Qiu, Y. Hua, Blind system identification, Proceedings of the IEEE 85 (8) (1997) 1310-1322. doi:10.1109/5.622507.

[45] [link].

URL http://www . wavsource.com/

[46] N. C. Geçkìnì, T. Güngen, H. Güngen, M. Etìşkol, Speech synthesis using am/fm sinusoids and band-pass noise, Signal Processing 8 (3) (1985) 339361. doi:10.1016/0165-1684(85)90111-2. 
[47] M. Wax, T. Kailath, Detection of signals by information theoretic criteria, IEEE Transactions on Acoustics, Speech, and Signal Processing 33 (2) (1985) 387-392. doi:10.1109/TASSP.1985.1164557.

[48] J. MacQueen, Some methods for classification and analysis of multivariate observations, in: Proceedings of the Fifth Berkeley Symposium on Mathematical Statistics and Probability, Volume 1: Statistics, University of California Press, Berkeley, Calif., 1967, pp. 281-297.

URL http://projecteuclid.org/euclid.bsmsp/1200512992

[49] G. Boudreaux-Bartels, T. Parks, Time-varying filtering and signal estimation using Wigner distribution synthesis techniques, IEEE Transactions on Acoustics, Speech, and Signal Processing 34 (3) (1986) 442-451. doi:10.1109/TASSP.1986.1164833.

[50] A. Aïssa-El-Bey, K. Abed-Meraim, Y. Grenier, Blind separation of underdetermined convolutive mixtures using their time-frequency representation, IEEE Transactions on Audio, Speech, and Language Processing 15 (5) (2007) 1540-1550. doi:10.1109/TASL.2007.898455.

[51] E. H. Moore, On the reciprocal of the general algebraic matrix, Bulletin of the American Mathematical Society 26 (9) (1920) 394-395. doi:10.1090/S0002-9904-1920-03322-7.

[52] W. Mu, M. G. Amin, Y. Zhang, Bilinear signal synthesis in array processing, IEEE Transactions on Signal Processing 51 (1) (2003) 90-100. doi:10.1109/TSP.2002.806577.

[53] I. E. Frank, R. Todeschini, The data analysis handbook, Vol. 14, Elsevier, 1994.

[54] H. Krim, M. Viberg, Two decades of array signal processing research: the parametric approach, IEEE Signal Processing Magazine 13 (4) (1996) 67-94. doi:10.1109/79.526899. 
[55] H. L. Van Trees, Detection, Estimation, and Modulation Theory, Vol. IV: Optimum Array Processing, John Wiley \& Sons, Inc., 2002.

[56] R. Schmidt, Multiple emitter location and signal parameter estimation, IEEE Transactions on Antennas and Propagation 34 (3) (1986) 276-280. doi:10.1109/TAP.1986.1143830.

[57] J. F. Cardoso, E. Moulines, Asymptotic performance analysis of directionfinding algorithms based on fourth-order cumulants, IEEE Transactions on Signal Processing 43 (1) (1995) 214-224. doi:10.1109/78.365301.

[58] A. Paulraj, R. Roy, T. Kailath, A subspace rotation approach to signal parameter estimation, Proceedings of the IEEE 74 (7) (1986) 1044-1046. doi:10.1109/PROC.1986.13583.

[59] P. Stoica, A. Nehorai, Performance comparison of subspace rotation and music methods for direction estimation, IEEE Transactions on Signal Processing 39 (2) (1991) 446-453. doi:10.1109/78.80828.

[60] B. Ottersten, M. Viberg, T. Kailath, Performance analysis of the total least squares esprit algorithm, IEEE Transactions on Signal Processing 39 (5) (1991) 1122-1135. doi:10.1109/78.80967.

[61] S. Ouelha, A. Aïssa-El-Bey, B. Boashash, Improving DOA estimation algorithms using high-resolution time-frequency distributions, IEEE Transactions on Signal Processing (accepted) (2017) .

[62] X. L. Xu, K. M. Buckley, Bias analysis of the music location estimator, IEEE Transactions on Signal Processing 40 (10) (1992) 2559-2569. doi:10.1109/78.157296.

[63] T. Ning, F.-S. Wei, Multichannel coherence analysis of helicopter vibrations, in: 6th International Conference on Signal Processing, Vol. 2, 2002, pp. 1774-1777. doi:10.1109/ICOSP.2002.1180146. 
[64] L. Koopmans (Ed.), The Spectral Analysis of Time Series, Vol. 22 of Probability and Mathematical Statistics, Academic Press, San Diego, 1995. doi:10.1016/B978-0-12-419250-8.

[65] L. B. White, B. Boashash, Cross spectral analysis of nonstationary processes, IEEE Transactions on Information Theory 36 (4) (1990) 830-835. doi:10.1109/18.53742.

[66] J. S. Bendat, A. G. Piersol (Eds.), Random Data, 4th Edition, Analysis and Measurement Procedures, John Wiley \& Sons, Inc., New Jersey, 2012. doi:10.1002/9781118032428.

[67] E. Pereda, R. Q. Quiroga, J. Bhattacharya, Nonlinear multivariate analysis of neurophysiological signals, Progress in Neurobiology 77 (1-2) (2005) 1-37. doi:10.1016/j.pneurobio.2005.10.003.

[68] K. J. Blinowska, Review of the methods of determination of directed connectivity from multichannel data, Medical \& Biological Engineering \& Computing 49 (5) (2011) 521—529. doi:10.1007/s11517-011-0739-x.

[69] S. Assous, B. Boashash, Evaluation of the modified s-transform for timefrequency synchrony analysis and source localisation, EURASIP Journal on Advances in Signal Processing 2012 (1) (2012) 49. doi:10.1186/16876180-2012-49.

[70] M. Rosenblum, A. Pikovsky, J. Kurths, Phase synchronization of chaotic oscillators, Physical Review Letters 76 (1996) 1804-1807. doi:10.1103/PhysRevLett.76.1804.

[71] A. Pikovsky, M. Rosenblum, J. Kurths, Phase synchronization in regular and chaotic systems, International Journal of Bifurcation and Chaos 10 (10) (2000) 2291-2305. doi:10.1142/S0218127400001481.

[72] B. Boashash, Estimating and interpreting the instantaneous frequency of a signal. i. fundamentals, Proceedings of the IEEE 80 (4) (1992) 520-538. doi:10.1109/5.135376. 
[73] S. Aviyente, E. M. Bernat, W. S. Evans, S. R. Sponheim, A phase synchrony measure for quantifying dynamic functional integration in the brain, Human Brain Mapping 32 (1) (2011) 80-93. doi:10.1002/hbm.21000.

[74] A. Omidvarnia, G. Azemi, P. B. Colditz, B. Boashash, A time-frequency based approach for generalized phase synchrony assessment in nonstationary multivariate signals, Digital Signal Processing 23 (3) (2013) 780 - 790. doi:10.1016/j.dsp.2013.01.002.

[75] J.-P. Lachaux, E. Rodriguez, J. Martinerie, F. J. Varela, Measuring phase synchrony in brain signals, Human Brain Mapping 8 (4) (1999) 194-208.

[76] S. Aviyente, A. Y. Mutlu, A time-frequency-based approach to phase and phase synchrony estimation, IEEE Transactions on Signal Processing 59 (7) (2011) 3086-3098. doi:10.1109/TSP.2011.2144589.

[77] F. Tadel, S. Baillet, J. Mosher, D. Pantazis, R. Leahy, Brainstorm: A userfriendly application for MEG/EEG analysis, Computational Intelligence and Neuroscience 2011 (879716) (2011) 13. doi:10.1155/2011/879716.

[78] Brainstorm toolbox.

URL http://neuroimage.usc.edu/brainstorm

[79] A. Gramfort, T. Papadopoulo, E. Olivi, M. Clerc, OpenMEEG: opensource software for quasistatic bioelectromagnetics, BioMedical Engineering OnLine 9 (1) (2010) 45. doi:10.1186/1475-925X-9-45.

[80] J. Kybic, M. Clerc, T. Abboud, O. Faugeras, R. Keriven, T. Papadopoulo, A common formalism for the integral formulations of the forward EEG problem, IEEE Transactions on Medical Imaging 24 (1) (2005) 12-28. doi:10.1109/TMI.2004.837363.

[81] R. Srinivasan, Methods to improve the spatial resolution of EEG, International Journal of Bioelectromagnetism 1 (1) (1999) 102-111. 
[82] C. Grova, J. Daunizeau, J.-M. Lina, C. Bénar, H. Benali, J. Gotman, Evaluation of EEG localization methods using realistic simulations of interictal spikes, NeuroImage 29 (3) (2006) 734-753. doi:10.1016/j.neuroimage.2005.08.053.

[83] A. Liu, J. Hahn, G. Heldt, R. Coen, Detection of neonatal seizures through computerized EEG analysis, Electroencephalography and Clinical Neurophysiology 82 (1) (1992) 30-37. doi:10.1016/0013-4694(92)90179-L.

[84] J. Gotman, D. Flanagan, B. Rosenblatt, A. Bye, E. Mizrahi, Evaluation of an automatic seizure detection method for the newborn EEG, Electroencephalography and Clinical Neurophysiology 103 (3) (1997) 363-369. doi:10.1016/S0013-4694(97)00005-2.

[85] S. Faul, G. Boylan, S. Connolly, L. Marnane, G. Lightbody, An evaluation of automated neonatal seizure detection methods, Clinical Neurophysiology 116 (7) (2005) 1533-1541. doi:10.1016/j.clinph.2005.03.006.

[86] H. Hassanpour, M. Mesbah, B. Boashash, Time-frequency based newborn EEG seizure detection using low and high frequency signatures, Physiological Measurement 25 (4) (2004) 935. doi:10.1088/0967-3334/25/4/012.

[87] B. Boashash, L. Boubchir, G. Azemi, A methodology for time-frequency image processing applied to the classification of non-stationary multichannel signals using instantaneous frequency descriptors with application to newborn EEG signals, EURASIP Journal on Advances in Signal Processing 2012 (1) (2012) 117. doi:10.1186/1687-6180-2012-117.

[88] M. A. Latif, S. Sanei, J. Chambers, L. Spyrou, Partially constrained blind source separation for localization of unknown sources exploiting nonhomogeneity of the head tissues, The Journal of VLSI Signal Processing Systems for Signal, Image, and Video Technology 49 (2) (2007) 217-232. doi:10.1007/s11265-007-0075-4. 
[89] R. Grech, T. Cassar, J. Muscat, K. P. Camilleri, S. G. Fabri, M. Zervakis, P. Xanthopoulos, V. Sakkalis, B. Vanrumste, Review on solving the inverse problem in EEG source analysis, Journal of NeuroEngineering and Rehabilitation 5 (1) (2008) 25. doi:10.1186/1743-0003-5-25.

[90] S. Baillet, J. C. Mosher, R. M. Leahy, Electromagnetic brain mapping, IEEE Signal Processing Magazine 18 (6) (2001) 14-30. doi:10.1109/79.962275.

[91] M. Hämäläinen, R. Hari, R. J. Ilmoniemi, J. Knuutila, O. V. Lounasmaa, Magnetoencephalography: theory, instrumentation, and applications to noninvasive studies of the working human brain, Reviews of Modern Physics 65 (1993) 413-497. doi:10.1103/RevModPhys.65.413.

[92] J. C. de Munck, B. W. van Dijk, H. Spekreijse, Mathematical dipoles are adequate to describe realistic generators of human brain activity, IEEE Transactions on Biomedical Engineering 35 (11) (1988) 960-966. doi:10.1109/10.8677.

[93] M. A. Latif, S. Sanei, J. Chambers, L. Shoker, Localization of abnormal EEG sources using blind source separation partially constrained by the locations of known sources, IEEE Signal Processing Letters 13 (3) (2006) 117-120. doi:10.1109/LSP.2005.862622.

[94] P. K. Banerjee, R. Butterfield, Boundary element methods in engineering science, Vol. 17, McGraw-Hill, London, UK, 1981.

[95] N. G. Gençer, I. O. Tanzer, Forward problem solution of electromagnetic source imaging using a new BEM formulation with high-order elements, Physics in Medicine and Biology 44 (9) (1999) 2275-2287.

[96] O. C. Zienkiewicz, R. L. Taylor, R. L. Taylor, The finite element method, Vol. 3, McGraw-Hill, London, UK, 1977. 
[97] Y. C. Zhang, S. A. Zhu, B. He, A second-order finite element algorithm for solving the three-dimensional EEG forward problem, Physics in Medicine and Biology 49 (13) (2004) 2975-2987.

[98] A. P. Bagshaw, A. D. Liston, R. H. Bayford, A. Tizzard, A. P. Gibson, A. Tidswell, M. K. Sparkes, H. Dehghani, C. D. Binnie, D. S. Holder, Electrical impedance tomography of human brain function using reconstruction algorithms based on the finite element method, NeuroImage 20 (2) (2003) 752-764. doi:10.1016/S1053-8119(03)00301-X.

[99] F. Darvas, D. Pantazis, E. Kucukaltun-Yildirim, R. Leahy, Mapping human brain function with MEG and EEG: methods and validation, NeuroImage 23, Supplement 1 (2004) S289-S299, mathematics in Brain Imaging. doi:10.1016/j.neuroimage.2004.07.014.

[100] M. D. Vos, W. Deburchgraeve, P. Cherian, V. Matic, R. Swarte, P. Govaert, G. Visser, S. V. Huffel, Automated artifact removal as preprocessing refines neonatal seizure detection, Clinical Neurophysiology 122 (12) (2011) 2345 - 2354. doi:10.1016/j.clinph.2011.04.026.

[101] C. M. Michel, M. M. Murray, G. Lantz, S. Gonzalez, L. Spinelli, R. G. de Peralta, EEG source imaging, Clinical Neurophysiology 115 (10) (2004) 2195 - 2222. doi:10.1016/j.clinph.2004.06.001.

[102] K. Sekihara, S. S. Nagarajan, D. Poeppel, S. Miyauchi, N. Fujimaki, H. Koizumi, Y. Miyashita, Estimating neural sources from each time-frequency component of magnetoencephalographic data, IEEE Transactions on Biomedical Engineering 47 (5) (2000) 642-653. doi:10.1109/10.841336.

[103] M. A. Klados, C. L. Papadelis, P. D. Bamidis, REG-ICA: A new hybrid method for EOG artifact rejection, in: 2009 9th International Conference on Information Technology and Applications in Biomedicine, 2009, pp. 1-4. doi:10.1109/ITAB.2009.5394295.

URL http://www.mklados.com/regica.html 
[104] Z. Akalin Acar, S. Makeig, Effects of forward model errors on EEG source localization, Brain Topography 26 (3) (2013) 378-396. doi:10.1007/s10548-012-0274-6.

[105] B. Boashash, S. Ouelha, An improved design of high-resolution quadratic time-frequency distributions for the analysis of nonstationary multicomponent signals using directional compact kernels, IEEE Transactions on Signal Processing 65 (10) (2017) 2701-2713. doi:10.1109/TSP.2017.2669899. 